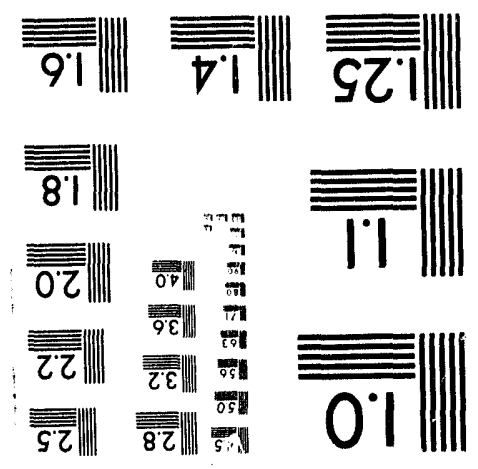



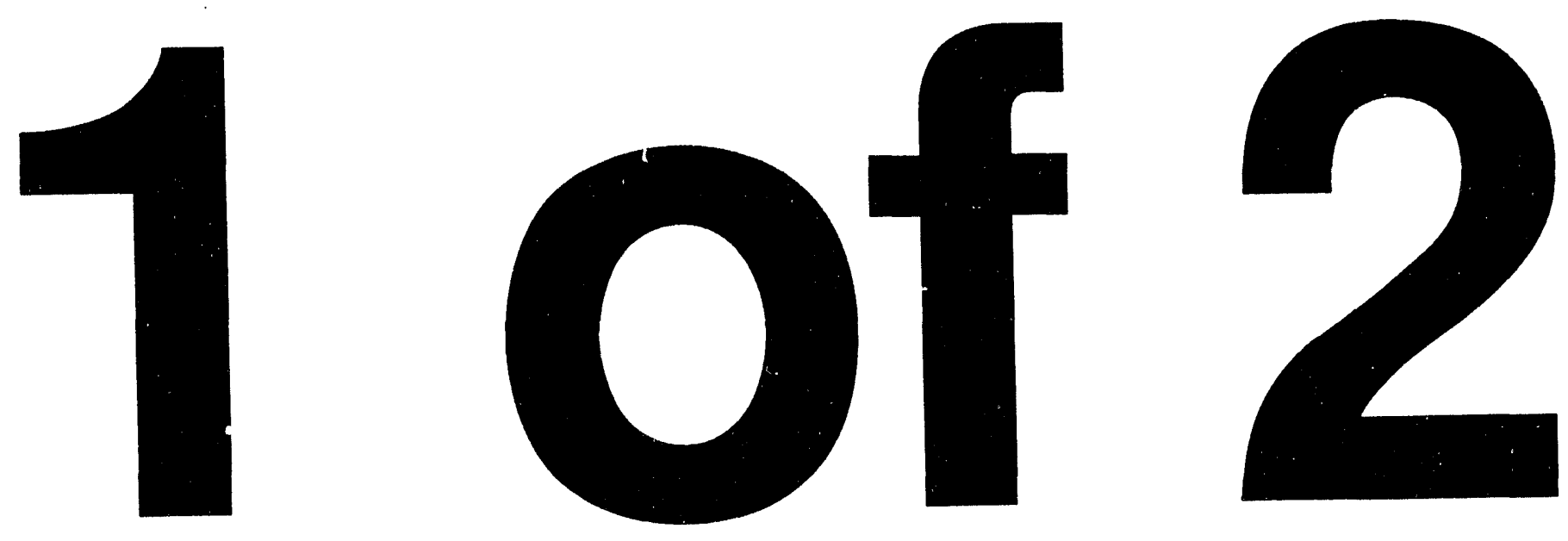


\title{
Investigations into Ultraviolet Matrix-assisted laser Desorption
}

\author{
by Theodore Heise
}

\author{
thesis submitted to Iowa State University \\ Ames Laboratory, U. S. DOE \\ Iowa State University \\ Ames, Iowa 50011
}

Date Transmitted: July 1993

\author{
Prepared for the U. S. Department of Energy \\ Under contract no. W-7405-eng-82.
}

\begin{abstract}
DISCLAIMER
This report was prepared as an account of work sponsored by an agency of the United States Government. Neither the United States Government nor any agency thereof, nor any of their employees, makes any warranty, express or implied, or assumes any legal liability or responsibility for the accuracy, completeness, or usefulness of any information, apparatus, product, or process disclosed, or represents that its use would not infringe privately owned rights. Reference herein to any specific commercial product, process, or service by trade name, trademark, manufacturer, or otherwise does not necessarily constitute or imply its endorsement, recommendation, or favoring by the United States Government or any agency thereof. The views and opinions of authors expressed herein do not necessarily state or reflect those of the United States Government or any agency thereof.
\end{abstract}


Investigations into ultraviolet

matrix-assisted laser desorption

Theodore William Heise

Under the supervision of Edward S. Yeung

From the Department of Chemistry

Iowa State University

Matrix-assisted laser desorption (MALD) is a technique for converting large biomolecules into gas phase ions. Some characteristics of the commonly used UV matrices are determined. Solubilities in methanol range from 0.1 to $0.5 \underline{\mathrm{M}}$. Solid phase absorption spectra are found to be similar to solution, but slightly red-shifted. Acoustic and quartz crystal microbalance signals are investigated as possible means of UV-MALD quantitation. Evidence for the existence of desorption thresholds is presented. Threshold values are determined to be in the range of 2 to $3 \mathrm{MW} / \mathrm{cm}^{2}$. A transient imaging technique based on laser-excited fluorescence for monitoring MALD plumes is described. Sensitivity is well within the levels required for studying matrixassisted laser desorption, where analyte concentrations are significantly lower than those in conventional laser desorption. Results showing the effect of film morphology, particularly film thickness, on plume dynamics are presented. In particular, MALD plumes from thicker films tend to exhibit higher axial velocities. Fluorescent labeling of protein and of DNA is used to allow imaging of their UV-MALD generated plumes. Integrated concentrations are available with respect to time, making it possible to assess 
the rate of desorption and the rate of fragmentation. The spatial and temporal distributions are important for the design of secondary ionization schemes to enhance ion yields and for the optimization of ion collection in time-of-flight MS instruments to maximize resolution. Such information could also provide insight into whether ionization is closely associated with the desorption step or whether it is a result of subsequent collisions with the mairix gas (e.g., proton transfer). Although the present study involves plumes in a normal atmosphere, adaptation to measurements in vacuum (e.g., inside a mass spectrometer) should be straightforward. 
Investigations into ultraviolet

matrix-assisted laser desorption

by

Theodore William Heise

A Dissertation Submitted to the

Graduate Faculty in Partial Fulfillment of the

Requirements for the Degree of

DOCTOR OF PHILOSOPHY

Department: Chemistry

Major: Analytical Chemistry

Approved:

$\frac{\sum .5 \text { Meng }}{\text { In Charge of Major Work }}$

For the Major Department

For the Graduate College

Iowa State University

Ames, Iowa 
TABLE OF CONTENTS

CHAPTER 1. INTRODUCTION $\ldots \ldots \ldots \ldots \ldots \ldots \ldots \ldots \ldots$

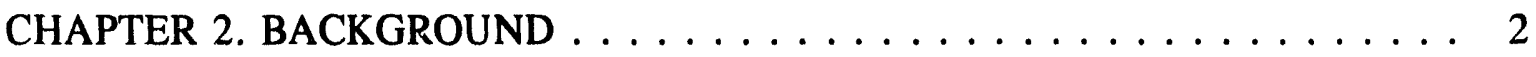

General Laser Desorption . . . . . . . . . . . . . . . . 2

Matrix-assisted Laser Desorption . . . . . . . . . . . . . . . 4

Desorption Geometries . . . . . . . . . . . . . . . . . . . . . . 14

Experimental Design . . . . . . . . . . . . . . . . 15

CHAPTER 3. CHARACTERIZATION OF MATRICES AND DYES $\ldots \ldots \ldots 18$

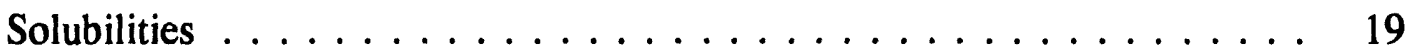

Sample Preparation .......................... 19

Film preparation methods . . . . . . . . . . . . . 19

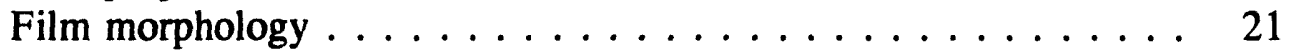

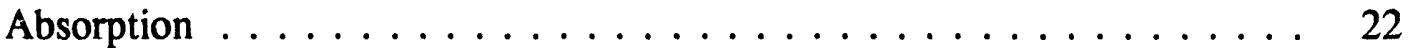

Method .......................... 22

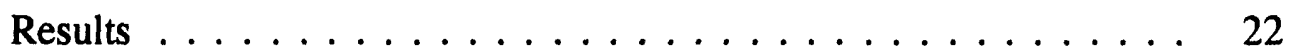

CHAPTER 4. QUANTITATION OF REMOVAL $\ldots \ldots \ldots \ldots \ldots$

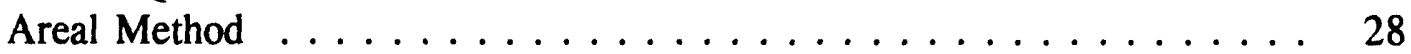

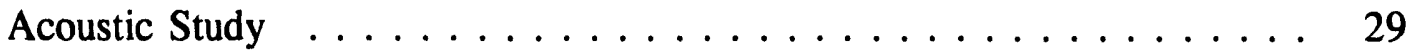

Quartz Crystal Microbalance ................. 36

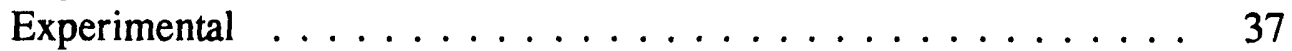

Results .......................... 37

CHAPTER 5. DESORPTION THRESHOLDS $\ldots \ldots \ldots \ldots \ldots \ldots \ldots$

CHAPTER 6. INTRODUCTION TO PLUME IMAGING $\ldots \ldots \ldots \ldots \ldots$

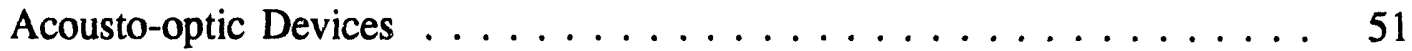

Imaging Background $\ldots \ldots \ldots \ldots \ldots \ldots \ldots \ldots \ldots$

CHAPTER 7. ABSORPTION STUDY $\ldots \ldots \ldots \ldots \ldots \ldots \ldots \ldots \ldots$

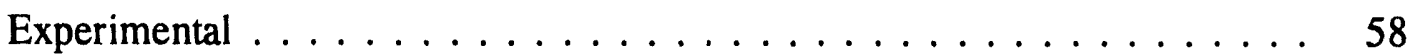

Results ...................... 59

CHAPTER 8. FLUORESCENCE PLUME IMAGING $\ldots \ldots \ldots \ldots \ldots$

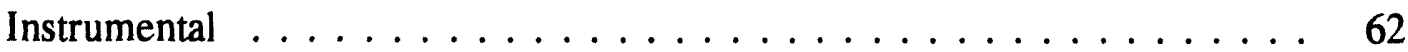

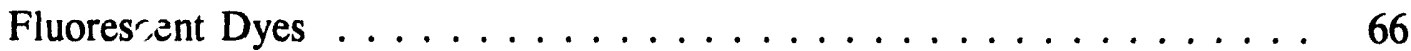

Reagents and sample preparation $\ldots \ldots \ldots \ldots \ldots 6$

Results ........................66 66

Dye Labeled Proteins $\ldots \ldots \ldots \ldots \ldots \ldots \ldots \ldots$ 


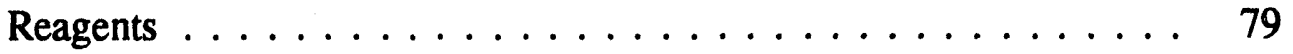

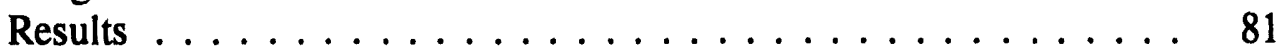

Dye Labeled DNA $\ldots \ldots \ldots \ldots \ldots \ldots \ldots \ldots \ldots$

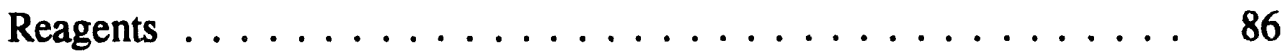

Results ....................... 86

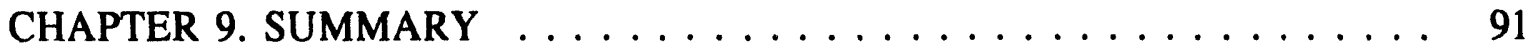

REFERENCES CITED . . . . . . . . . . . . . . . . . . . . 93

ACKNOWLEDGEMENTS $\ldots \ldots \ldots \ldots \ldots \ldots \ldots$ 


\section{CHAPTER 1. INTRODUCTION}

This dissertation is organized in traditional format. It begins with a general introduction of the area under study. Experimental details common to the whole work come next, followed by the results. Results are arranged roughly in order of increasing degree of complexity and difficulty. Details specific to particular experiments are introduced in the pertinent sections.

Portions of this dissertation have been published or are in the process of being published. To satisfy the copyright requirements of the respective journals the following credit lines are included:

Portions of this dissertation are reprinted with permission from:

Heise, T. W.; Yeung, E. S. Anal. Chem. 1992, 64(18), 2175-2179. 01992 American Chemical Society

Although a majority of recent doctoral candidates from this research group have organized their theses with each published paper constituting a chapter, it is my belief that the readability and utility of this work is enhanced by using a traditional format. 


\section{CHAPTER 2. BACKGROUND}

\section{General Laser Desorption}

Ever since the first laser was devised, ${ }^{1}$ scientists and non-scientists alike have been fascinated by these unique light sources. A great deal of scientific research has been devoted to developing uses for lasers. Very early on, researchers made use of the high fluences available with pulsed lasers to vaporize solid phase material. No doubt this curiosity has been driven, at least in part, by man's desire to control the world around him. A further impetus may be his innate fascination with fire and with explosions.

Laser vaporization of solids is generally divided loosely into two classes based on the regime of laser irradiance. At irradiances greater than $\sim 10^{8} \mathrm{~W} / \mathrm{cm}^{2}$ the process is usually termed laser ablation. Laser desorption is most commonly used to refer to processes occurring at irradiances of less than $\sim 10^{8} \mathrm{~W} / \mathrm{cm}^{2}$. Generally irradiances of less than $10^{6} \mathrm{~W} / \mathrm{cm}^{2}$ produce little change in a solid material. For a discussion of these interactions the reader is referred to S. M. Kimbrell's Ph.D. dissertation. ${ }^{2}$ Over time lasers have come to be widely used as a versatile means of interrogating solid phase material. One extremely useful application of this process has been as a means of solids sampling for mass spectral analysis.

Mass spectrometry (MS), based on the separation of charged particles in a magnetic field, delivers information about molecular weights of atoms, molecules, and molecular fragments. MS analysis requires that the sample be amenable to conversion 
to gas phase ions. For samples that are already gaseous or are condensed phase with a significant vapor pressure, this requirement presents no great difficulty. For solid samples with no significant vapor pressure, however, conversion to gas phase can be a problem, particularly if molecular information about the sample is desired. Several approaches that have been effective in producing intact biomolecules in the gas phase include field desorption, ${ }^{3}{ }^{252} \mathrm{Cf}$ plasma desorption, ${ }^{4}$ secondary ion $\mathrm{MS},{ }^{5}$ thermo- ${ }^{6}$ and electrospray, ${ }^{7}$ and laser desorption (LD) ${ }^{8}$

The amount of energy required to convert a condensed phase sample to gas phase typically is also enough to break intramolecular bonds. A significant portion of the energy deposited in the sample molecules manifests itself as vibrations within the molecule. Whell these vibrations are more energetic than the strength of the intramolecular bonds, the bonds break -- resulting in shattering or fragmentation of the molecule. One useful characteristic of laser desorption is that it is capable of vaporizing solid samples while minimizing fragmentation of molecules. ${ }^{9}$ This property is based on the pulsed laser's ability to deliver its energy to a sample very rapidly. The result can be explained loosely in these terms. ${ }^{10}$ Vaporization and decomposition are processes which can be described using Arrhenius rate equations. For first order kinetics, $\log$-log plots of reciprocal of temperature versus rate constant are linear. The two processes most probably have Arrhenius plots with differing slopes which will intersect at some temperature. At temperatures below the intersection decomposition will be favored, while vaporization is favored at higher temperatures. The rapid temperature rise induced by the laser pulse causes the sample to reside in the 
temperature range favorable to decomposition for a very short time. The sample components are vaporized before they have time to start vibrating. Because vibrations are not energized sufficiently to break bonds, the molecule stays intact. Even though laser vaporization was a major stride forward in MS analysis of large molecules, it is not without its shortcomings. LD is not capable of vaporizing intact biomolecules of very high molecular weight. In addition, even with the use of a 10 nanosecond pulse from a frequency doubled Nd:YAG laser, significant sample destruction has been observed. ${ }^{11}$ With the continually increasing interest in biological systems, there is also great interest in developing methods for their analysis. Biological systems with their great degree of complexity and very large molecules offer major challenges to the mass spectroscopist. The recent discovery and development of matrix-assisted laser desorption has been a significant breakthrough in this area and is enabling the mass spectrometrist to meet these challenges.

\section{Matrix-assisted Laser Desorption}

In brief, matrix-assisted laser desorption (MALD) is the method of dispersing the biopolymer of interest in a large excess of a matrix. The mixture is irradiated with a laser pulse and vaporizes. Analyte ions are formed and then detected by mass spectrometry. One important criterion for choosing a matrix is that it absorb strongly at the wavelength of the desorption laser. Although the technique has been demonstrated for a wide range of desorption wavelengths, the most widely used is the ultraviolet (UV). The MALD technique is not fully understood, but has rather been developed empirically. In order to better understand the dynamics of the process more 
information is needed.

Matrix-assisted laser desorption is a fairly recent innovation. As early as 1985 references were made to MALD. ${ }^{12}$ The main breakthrough did not take place till several years later with the discovery of the first practical matrix. ${ }^{13}$ The experiment consisted of dispersing a protein sample in a thousand-fold molar excess of nicotinic acid matrix. The sample mixture was then subjected to a UV fourth harmonic pulse $(266 \mathrm{~nm})$ from a Nd:YAG laser. A strong MS signal from the protein pseudomolecular ion was observed. At about the same time Tanaka and co-workers published an article ${ }^{14}$ describing a very similar technique. In this second approach, proteins and finely divided silver are intimately dispersed in a matrix of glycerol. The common theme is that in both methods the analyte does not absorb the impinging laser irradiation -- other components of the sample mixture do. The desorption laser pulse energy is transferred either directly or indirectly to the sample matrix. In Hillenkamp's work, the matrix itself directly absorbs the laser energy. In the case of Tanaka's work, the silver absorbs the light and couples its energy to the glycerol matrix. The result in both cases is a vaporization of the matrix with the analyte being swept along into the gas phase.

A great deal of research activity has been directed towards increasing both the utility and the understanding of MALD. The results of this work have led to demonstration of MALD under quite a variety of conditions. Examples include variations in desorption laser wavelength, matrix material, and mass spectrometric detection method. 
The MALD effect has been demonstrated at numerous desorption laser wavelengths. As mentioned previously, the original work used the Nd:YAG fourth harmonic. Other wavelengths used include the $10.6 \mu \mathrm{m}$ output from a TEA-CO laser, ${ }^{15}$ the $2.94 \mu \mathrm{m}$ output from an Er-YAG laser, ${ }^{16}$ and the Nd:YAG $1064 \mathrm{~nm}$ fundamental, ${ }^{17} 532 \mathrm{~nm}$ second harmonic, ${ }^{17,18}$ and $355 \mathrm{~nm}$ third harmonic ${ }^{19}$ outputs. Also, excimer lasers operating at $353 \mathrm{~nm},{ }^{20} 307 \mathrm{~nm},{ }^{21} 248 \mathrm{~nm}$, and $193 \mathrm{~nm}^{22}$ wavelengths and the nitrogen $337 \mathrm{~nm}$ output ${ }^{23}$ have been used.

A wide variety of matrices have been used as well. These can be divided into solid or liquid matrices. Solid matrices in addition to the original nicotinic acid ${ }^{13}$ include a variety of cinnamic acid derivatives, ${ }^{24} 3$-hydroxypicolinic acid, ${ }^{25}$ and even rhodamine 6G. ${ }^{26}$ In Tanaka's "ultra fine metal plus liquid matrix method" the matrix is glycerol. ${ }^{14}$ 3-nitrobenzyl alcohol ${ }^{27}$ and frozen aqueous solutions ${ }^{28}$ are other liquid matrices. It should be pointed out that some liquid matrices require a second component to couple the laser light energy into the matrix as the matrices themselves are not strongly absorbing. The glycerol method of Tanaka relies on finely divided silver. In the cryogenic aqueous approach the absorber is less intimately connected with the sample. Laser energy is coupled into the sample via a copper cold finger upon which the sample is deposited. The absorber is actually the supporting substrate. This substrate approach has been recently shown to work for solid matrices as well, ${ }^{29}$ although the authors of that paper express the belief that the desorption mechanism is distinctly different from that of MALD. For the sake of thoroughness, it is mentioned here that even attempts at two-component matrices have been made. ${ }^{18}$ 
The reasons one compound makes a satisfactory MALD matrix and another very similar compound does not are not clear. ${ }^{30}$ Some general properties of useful MALD matrices include:

1) strong absorption of the irradiating wavelength,

2) solubility in appropriate solvents,

3) reasonable vapor pressure (often easily sublimeable),

4) proper reactivity (i.e., ion formation),

5) supply of proton pool, ${ }^{31}$

6) isolation of analyte molecules. ${ }^{32}$

Otherwise useful organic acid matrices have been ineffective when used from solutions of high $\mathrm{pH}^{33}$ Because the salt form has a lower vapor pressure, this supports the notion of a volatility requirement. The abundance of protons in an organic acid matrix may play a critical part in ion formation. A range of matrix to analyte ratios have been used, but the best results typically are generated within a rather narrow range of ratios, typically around a 1000 -fold molar excess of matrix. With larger ratios the decreasing amount of analyte present becomes difficult to detect. With smaller ratios, the analyte molecules are no longer isolated and fragmentation becomes a problem. To overcome the severe wavelength restriction associated with the original nicotinic acid matrix, Beavis and Chait conducted extensive work ${ }^{34}$ to determine matrices suitable for wavelengths longer than $266 \mathrm{~nm}$.

Because of the temporally discrete sample sampling process, MALD is particularly well-suited to time-of-flight (TOF) mass spectrometry. Although the great 
majority of MALD-MS in fact uses TOF mass analysis, there have been numerous attempts to apply the MALD technique to other MS techniques. A brief sampling includes the use of Fourier transform MS,${ }^{35}$ quadrupole ion-trap $\mathrm{MS},{ }^{36}$ and magnetic sector MS. ${ }^{20}$

The majority of MALD-MS work has been carried out in positive ion mode although negative ion spectra have been demonstrated. ${ }^{19}$ Single laser shots often yield useful spectra, but most commonly data from 10 to 100 shots are collected and averaged..$^{32}$

The technique characteristically produces single peak spectra. This single peak usually arises from a molecular or pseudo-molecular ion. A drawback of single peak spectra is that little structural information is obtained. The simplicity of the spectra does suggest a certain utility of MALD-MS for mixture analysis. MS/MS is difficult to

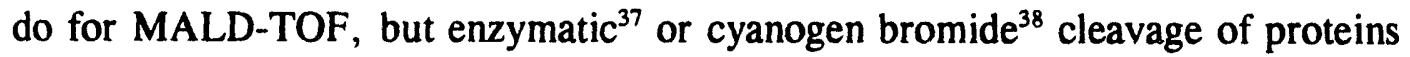
prior to MS analysis can yield some of the same information. Recently, metastable decay of large laser desorbed ions has been used to perform peptide sequencing. ${ }^{39}$ Resolution is generally rather poor, but mass accuracies approaching $0.01 \%$ can be realized for molecular masses up to $40 \mathrm{kDa} .{ }^{40}$ Poor resolution has been attributed to metastable decay of ions and formation of adducts. ${ }^{31}$ Countering the drawbacks are several strong positive features. First, the method produces useful mass spectra of large biopolymers. Second, it is very sensitive and quite rapid. Spengler and Kaufmann claim that UV-MALD outperforms gel electrophoresis by at least an order of magnitude in analysis time, sensitivity, and accuracy. ${ }^{31}$ Additionally, the method is 
extremely tolerant of sample matrix. Excellent mass spectra can be obtained even in the presence of high contaminant concentration. ${ }^{40}$ The consequence is that sample preparation need not be complicated or lengthy. Decent spectra have been obtained from complex samples with little or no pretreatment. As one example, Beavis and Chait have obtained spectra with strong peaks of high-mass ( $>40 \mathrm{kDa}$ ) components of bovine and human milk. ${ }^{38}$ This analysis was completed in less than 15 minutes. Spectra from protein at $10 \mu \underline{\mathrm{M}}$ concentration in $10 \%$ sodium dodecyl suifate have also been obtained. ${ }^{41}$

Quality and intensity of spectra are strongly affected by sample morphology. Sample preparation typically consists of mixing an analyte solution with a solution of matrix. A small volume of the mixture is deposited on a probe and dried under a stream of warm air. The prepared sample can take many forms ${ }^{32}$ ranging from rather homogeneous amorphous layers, to closely packed microcrystallites, or loosely scattered needles or some combination. The variations are dependent on matrix type and sample preparation method. Samples often contain a higher matrix concentration in a ring around the edge with analyte dispersed more uniformly throughout the center. ${ }^{42}$ Films are typically rather heterogeneous. MS peak intensity varies widely with sampling position ${ }^{43}$ with stronger signals coming from irradiation of film edges. ${ }^{42}$ In reference 43 , the authors also report that more concentrated samples (presumably also thicker) give larger signals. A number of workers have reported that MALD directly from individual crystallites produces the strongest signals..$^{32,41}$ In fact, single crystals grown from saturated solution have produced excellent spectra. ${ }^{41}$ It has been suggested 
that materials which degrade MALD signals do so because they interfere with crystallization of the matrix/analyte mixture. ${ }^{44}$ It appears that an intimate mixture of analyte and matrix is important. However, production of excellent MALD mass spectra from the liquid matrix 3-nitrobenzyl alcohol refutes the notion that a perfect lattice structure may be a MALD requirement. ${ }^{27}$

For the UV absorbing matrices, the role of the substrate appears to be of minor or no importance. This has been demonstrated by MALD from crystals of sample distributed on a plastic-coated electron microscope grid. ${ }^{45}$

The MALD process has been demonstrated to be a collective event with little or no signal present below a certain threshold laser irradiance. ${ }^{21}$ A range of threshold values have been reported, but most are in the 1 to $10 \mathrm{MW} / \mathrm{cm}^{2}$ range.

At this point it would be good to discuss the nomenclature. Desorption is somewhat of a misnomer. The process, although relatively poorly understood, is known to be a bulk process rather than a monolayer type phenomenon as would be implied when surface scientists speak of desorption. ${ }^{46}$ The actual mechanism is not known, but may be an explosive ejection or a bulk vaporization of material. Furthermore, there is some inconsistency in use of the MALD acronym itself with some groups referring to the process as matrix-assisted laser desorption/ionization (MALDI). This author favors the use of MALD for two reasons. First of all, it is uncertain at which time and by what mechanism ionization actually occurs. Ionization may or may not be directly associated with laser desorption. Second, for this work, the actual ionization step is somewhat irrelevant. 
As mentioned before, the mechanism of the process is not well understood. A number of models have been proposed for the MALD process.

Vertes and coworkers have set forth a model they term the homogeneous bottleneck. ${ }^{47}$ This model describes the matrix as a host and the analyte as a guest. Homogeneity enters into the model in the assumption that the illuminated portion of the mixture comes to a uniform energy density. The term bottleneck refers to an energy bottleneck. It is used to describe the poor coupling between guest molecule internal modes and lattice vibrations. The bottleneck dictates that the rate of sublimation of the host is significantly greater than the rate of energy transfer to the guest. The result is desorbed guest molecules which are vibrationally cold. Another point these researchers make is that expansive cooling by the matrix may minimize fragmentation.

Johnson and Sundqvist ${ }^{48}$ have compared MALD to fast-ion induced sputtering. They propose that there are two components to the ejection mechanism: 1) a sublimation-like process in which material leaves, "according to the local surface temperature", and 2) a pressure-pulse process in which a pressure gradient exceeding some energy threshold causes ejection. The authors refer to these two parts as a thermal spike and a pressure pulse which together comprise a complete hydrodynamic model.

Rapid heating seems to be a vital component of any model. For LD, it has been said that rapid heating desorbs molecules before they have time to decompose. ${ }^{9}$ Vertes states that the nanosecond lasers typically used in UV-MALD can easily produce $10^{10}$ to $10^{11} \mathrm{~K} / \mathrm{s}$ surface heating rates. ${ }^{49}$ 
Spallation, the ejection of large relatively cold pieces of material, has been observed to occur. Williams and Nelson cite Nelson's unpublished thesis in which spallation is more pronounced with thicker films. ${ }^{50}$ For thinner films the MALD process appears to be more of an ablation process producing diffuse ejecta with a forward peaked angular distribution. Beavis and Chait, on the other hand, maintain that production of gas phase ions cannot be the result of removal of large pieces of cold solid material. ${ }^{51}$

For the more general case of laser desorption, some authors have proposed nonequilibrium processes such as explosive desorption ${ }^{52}$ or a shock wave. ${ }^{53}$ There is no immediately obvious reason why these processes might not also apply to MALD. One could compare a shock wave mechanism to Johnson and Sundqvist's pressure pulse model. In fact more recently, the concept of shock heating of the matrix material has been emphasized. ${ }^{50}$ In this paper the authors suggest the importance of rapid heating induced by a shock wave. The importance of a shock wave type of mechanism seems to be more pronounced in the case of backside desorption geometry. ${ }^{54}$ These workers also make reference to the concept of a pressure pulse and relace the process to the shock wave transfer of momentum seen in plasma desorption MS. Vertes also refers to the MALD process as a non-equilibrium phase transition. ${ }^{49}$ He notes that for a shock wave model to be viable, the compression wave velocity must exceed that of heat conduction.

Beavis and Chait have described the MALD process as consisting of a rapidly expanding gas jet following a rapid phase change of the solid matrix. ${ }^{s 1}$ 
As well as the ejection mechanism, the exact ionization process is not fully understood. It is clear that direct ionization from the LD step is not very efficient. Ion yields are generally $<1 \%$ at irradiances around $10^{6} \mathrm{~W} / \mathrm{cm}^{2}{ }^{.55}$ Hillenkamp actually estimates yields which are lower by as much as two orders of magnitude. ${ }^{56}$ Beavis and Chait point out that the intensity ratios of singly to doubly charged ions often change with changes in matrix. They cite this as evidence for believing the detailed protein matrix interaction is important. ${ }^{51}$ To further complicate matters, they show that ion production times are longer than the desorption laser pulse $(\sim 10 \mathrm{~ns})$. They conclude that either ions are produced from the surface for longer than the laser is on, or that ions are not immediately accelerated. It has been suggested that likely ionization mechanisms are proton transfer and cationization. ${ }^{57}$ The Hillenkamp group states that the matrix geometric isomer plays a role in the photochemistry of analyte ionization. ${ }^{33}$ Although there is a definite threshold for ion production, it is also shown that a significant decrease in ion signal accompanies an increase in irradiance. ${ }^{58}$ It has also been pointed out that ease of ionization decreases with increasing molecule size..$^{59}$

A significant amount of effort has been directed toward determining ion velocities. Again, there is not general agreement in the literature. There appears to be a significant difference between radial and axial velocities. Ens and coworkers have reported radial velocities about 20 times less than axial. ${ }^{21}$ Reported axial velocities range from as high as $1500 \mathrm{~m} / \mathrm{s},{ }^{60}$ to as low as $20 \mathrm{~m} / \mathrm{s},{ }^{61}$ with most values falling somewhere between these two extremes. ${ }^{50,62}$ Most evidence indicates that axial velocities are independent of analyte molecular weight. ${ }^{63}$ In attempts to measure kinetic 
energy, a number of workers have reported an energy deficit for molecular ions. Possible explanations ${ }^{63}$ for this phenomenon include:

1) collisions with other particles in the gas hinder acceleration of molecular ions through the extraction field,

2) neutral ejecta are ionized by collisions a distance above the surface,

3) surface charging may reduce the local field encountered by the ions.

In an excellent review, ${ }^{46}$ Sundqvist has concluded that the axial velocity of the ions is largely a function of supersonic expansion of the forward ejected matrix molecules.

Whatever the mechanism may be, one can certainly say MALD has proven to be of great utility in the MS analysis of otherwise intractable biopolymers. ${ }^{64}$ Peptides, ${ }^{65}$ proteins, ${ }^{66}$ oligosaccharides, ${ }^{67}$ oligonucleotides, ${ }^{25}$ and even double-stranded DNA approaching $20 \mathrm{kDa}^{68}$ have been converted to gas phase ions for mass analysis by this method. The greatest utility has been for molecular weight determinations of proteins with values of $250 \mathrm{kDa}$ being obtainable. ${ }^{69}$

Due to its unique capabilities MALD has received a great deal of attention in the mass spectrometric community. The rapidly growing popularity of MALD-TOF-MS is demonstrated by a recent report from PittCon $93 .^{70}$ In this report commercial MALD instrumentation is available from no fewer than four manufacturers -- including one with automated sample introduction. 


\section{Desorption Geometries}

In general two laser desorption geometries are possible -- frontside or backside. ${ }^{71}$ These are sometimes referred to as reflection and transmission geometries respectively ${ }^{45}$ In frontside desorption the irradiating beam is directed at the sample from a small angle about the surface normal. In backside geometry the beam is directed through a relatively transparent support, arriving at the sample where it is attached to the support. It has been shown that laser generated plumes can significantly attenuate the laser energy delivered to that surface. ${ }^{72}$ It is thought that back-side desorption tends to minimize plasma effects due to interaction of the desorption laser with the vaporized sample.

\section{Experimental Design}

A number of different experimental setups were used in the work discussed in this thesis. The desorption strategy was unchanged throughout most of the experiments and will be detailed here. Other experimental details will be described in the section appropriate to the work.

A nitrogen laser (Lambda Physik, EMG 101) was used for sample desorption. Two different desorption geometries were used. The desorption setup for the backside geometry is shown in Figure 1. A concave mirror was used to focus the pulses to a line. Measurements from burn spots on photographic film and vaporized samples showed the desorption pulse to be focused to $0.5 \times 6 . \mathrm{mm}$ giving an area of $3 \mathrm{~mm}^{2}$. There were several reasons for using this configuration. First, this focussing 


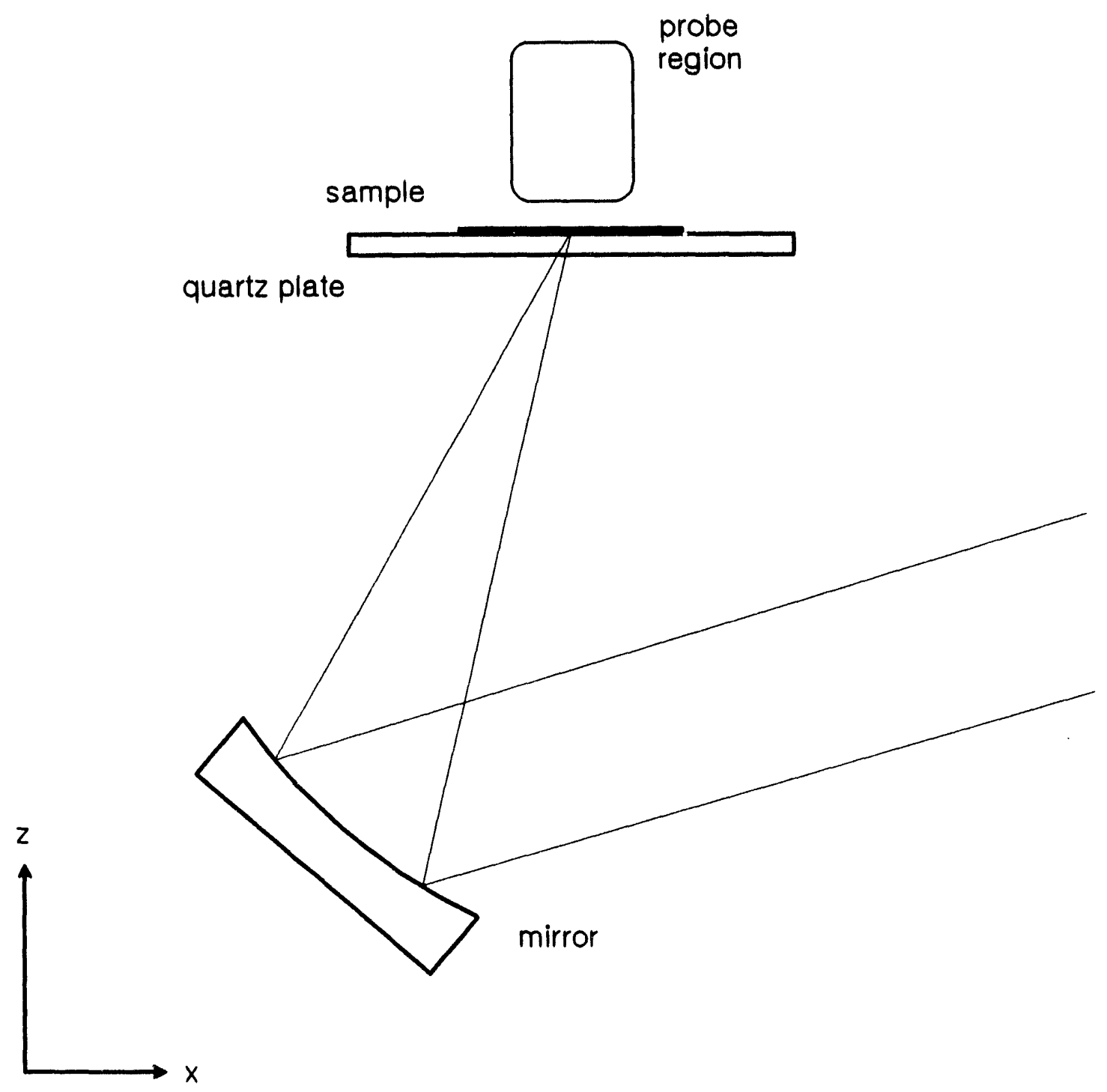

Figure 1. Block diagram of the desorption strategy with backside desorption illustrated 
arrangement was found to give the most uniform beam profile. Second, this setup was also found to yield the highest irradiance. Finally, a line was used rather than a point to increase the probe pathlength within the plume for the fluorescence studies. The laser was operated at low rep rates $(\sim 0.5 \mathrm{~Hz})$ with a shutter manually opened to admit a single laser pulse. Therefore each set of data collected was for a single desorption event. It should be stressed here that unlike the majority of MALD work, no signal averaging was done. Laser fluence was varied by placing glass plates at $45^{\circ}$ angles in the beam path. This allowed up to an order of magnitude beam attenuation in 15 to $20 \%$ increments.

Desorption of samples was carried out at atmospheric pressure without any special enclosures. The pulse energy was monitored by directing a partially reflected portion of the desorption beam to an energy probe (Laser Precision, Utica, NY, models Rj-7200 and Rjp734). Typical pulse ene:gies ranged from 1 to $4 \mathrm{~mJ}$ at the desorption site with pulse widths of $\sim 20 \mathrm{~ns}$.

Samples were prepared on quartz plates. Quartz absorbs very minimally at the desorption wavelength so MALD can be studied independently of substrate contributions. The efficiency of sample desorption was checked by visual inspection of the films using a microscope. 


\section{CHAPTER 3. CHARACTERIZATION OF MATRICES AND DYES}

Matrices were chosen based on previous utility in UV-MALD, and were obtained from Aldrich. The ferulic acid as received had a noticeable purple cast to it. Attempts were made to purify it by recrystallization from boiling water. ${ }^{73}$ Considerable effort was expended with very little change in appearance, so all matrices were used as received. The matrices are listed in Table I. All solutions were prepared in methanol unless otherwise noted.

Table I. Ultraviolet MALD matrices investigated

\begin{tabular}{lll}
\hline IUPAC name & common name & $\begin{array}{l}\text { MW } \\
(\mathrm{g} / \mathrm{mol})\end{array}$ \\
\hline 2-aminobenzoic acid & anthranilic acid & 137.14 \\
3,4-dihydroxycinnamic acid & caffeic acid & 180.16 \\
4-hydroxy-3-methoxycinnamic acid & ferulic acid & 194.19 \\
2,5-dihydroxybenzoic acid & gentisic acid & 154.12 \\
3,5-dimethoxy-4-hydroxycinnamic acid & sinapinic acid & 224.21 \\
\hline
\end{tabular}

Dyes were chosen based on molar absorptivity at both $337 \mathrm{~nm}$ and $488 \mathrm{~nm}$. Specifically it was desired to investigate dyes with both large and small absorbance coefficients at the desorption wavelength. The idea here was that differences in absorptivity might be observed as differences in dye destruction. Of course a strong 
absorbance at the fluorescence probe wavelength was essential.

The dyes chosen were coumarin 6 (C6) and coumarin 334 (C3), both obtained from E2stman, rhodamine 6G (R6G) from Exciton, and disodium fluorescein (UR) obtained from Lambda Physik. The dye solutions were prepared in methanol.

\section{Solubilities}

After investigating a number of solvents, it was found that methanol produced the best sample films. It was used in all cases. To generate the thickest possible films, saturated solutions of matrix were needed. Solubilities were determined by repetitively adding volumes of methanol to a known mass of sample until no solid remained. Matrix solubilities ranged between 0.1 and $0.5 \underline{\mathrm{M}}$. Ferulic and sinapinic acid solubilities were $0.5 \underline{\mathrm{M}}$. Caffeic acid was soluble up to about $0.25 \underline{\mathrm{M}}$. Gentisic acid had lower solublity at $0.1 \mathrm{M}$. The solublity of anthranilic acid was not determined.

Dye solubilities were found to be at least $10 \mathrm{mM}$ for R6G and UR. In order to keep within acceptable matrix:dye ratios, the possibility of preparing more concentrated solutions of those dyes was not pursued. C3 was found to have a maximum solubility of $5 \mathrm{~m} \underline{\mathrm{M}}$. C6 solubility was significantly less at $0.3 \mathrm{mM}$.

\section{Sample Preparation}

Film preparation methods

With the exception of the quartz crystal microbalance (QCM) work, samples were always prepared on $2 \times 2 \times 1 / 16^{\text {" }}$ quartz plates. Preparation of sample films 
received considerable study. In order to have a reliable estimate of the amount of material desorbed, the most niform sample films were desired. A number of methods of sample preparation were tried. These included casting from various solvents, casting both under atmospheric and reduced pressures, and room and elevated temperatures, spraying with a nebulizer, evaporating within a well, dipping, vapor deposition, painting, and spin-coating. Additionally various pre-treatments of the quartz surface were tried. These included acid washing, base washing, rinsing with a surfactant, and silanization. In the end, spin-coating from alcoholic solutions was found to give the most uniform films. This procedure was capable of producing uniform films up to a maximum thickness of $\sim 700 \mathrm{nmol} / \mathrm{cm}^{2}$. To produce thicker films uniformity was sacrificed and direct casting was used. For the acoustic studies, all samples were prepared by spin-coating $10 \mu \mathrm{L}$ aliquots of methanolic solutions. The spin-coater was home built and operated at $800 \mathrm{rpm}$. It produced films which were essentially circular and quite uniform. Matrix solutions of varying concentrations were used to obtain a range of film thicknesses. For the QCM experiment, sample films were cast by depositing $1 \mu \mathrm{L}$ aliquots directly onto the electrode surface. The films formed matched the electrode area nearly exactly. They were rather uniform albeit less so than with spin-coating. For most of the fluorescence work, thicker films were needed. Casting of films in air was used to get those thicker films, but at the expense of film uniformity. The air cast films had observable thicker crusts at the periphery.

Film thicknesses were determined from the concentration and volume of solution deposited. With this knowledge and the assumption of uniform film distribution, 
measurement of the area of the film allowed calculation of the film thickness. Implicit in the uniformity assumption is the further assumption that the dye was dispersed uniformly throughout the matrix.

Film morphology

Film thicknesses were estimated to vary over a range from several hundred $\mathrm{nm}$ to several $\mu \mathrm{m}$ thick. These estimates were based on the assumption of uniform matrix distribution and a density estimate of $\sim 1 \mathrm{~g} / \mathrm{mol}$.

Film morphology varied tremendously with sample prep method, analyte, and matrix. A great deal has been written about the variations in MALD sample morphology. In general, the ferulic acid solutions gave the most uniform films. In agreement with most of the literature reports, a thicker ring of sample formed at the edge of cast films. Films formed by spin-coating did not exhibit this edge effect and were nicely uniform. Although they were too thin to give much information under a microscope, macroscopically they were extremely even looking. The error bars in Figure 3 give a good sense of the film uniformity. Another estimation of film uniformity was available from interference effects within the films. Reflected room light was limited to mostly a single color per film. Supposing that the interference is first order, this would suggest a thickness variation of no more than $10 \%$.

An observable change in ferulic acid films occurred over time. The films, which were initially transparent, became opaque with the passage of time. This time period varied from 10 minutes to an hour. It is possible that some sort of phase 
transition was taking place. The change in appearance was speeded when the plates were warmed. The caffeic acid samples were typically opaque immediately upon drying. The other matrices typically produced transparent films which did not become opaque. Anthranilic acid tended to coat the quartz surface quite unevenly.

\section{Absorption}

As mentioned previously, one important characteristic of a useful MALD matrix is a strong absorption of the desorption laser wavelength. To help define this important parameter, further study was warranted.

\section{Method}

Absorbance spectra and absorptivity constants were determined using a Hewlett Packard photodiode array spectrophotometer. Solution spectra were taken in methanol solution. The films were produced by spin-coating onto quartz plates and measured by simply placing the plate in the spectrometer with the sample oriented perpendicular to the beampath.

\section{Results}

Absorbance spectra for both solution and film samples of the five matrices investigated are displayed in Figure 2. A trend common to all samples is that the absorbance maximum is shifted to longer wavelengths for the film. Otherwise the spectra are largely unchanged. Molar absorptivities for films and solutions at $338 \mathrm{~nm}$ were also determined for each matrix. The values are listed in Table II. An 


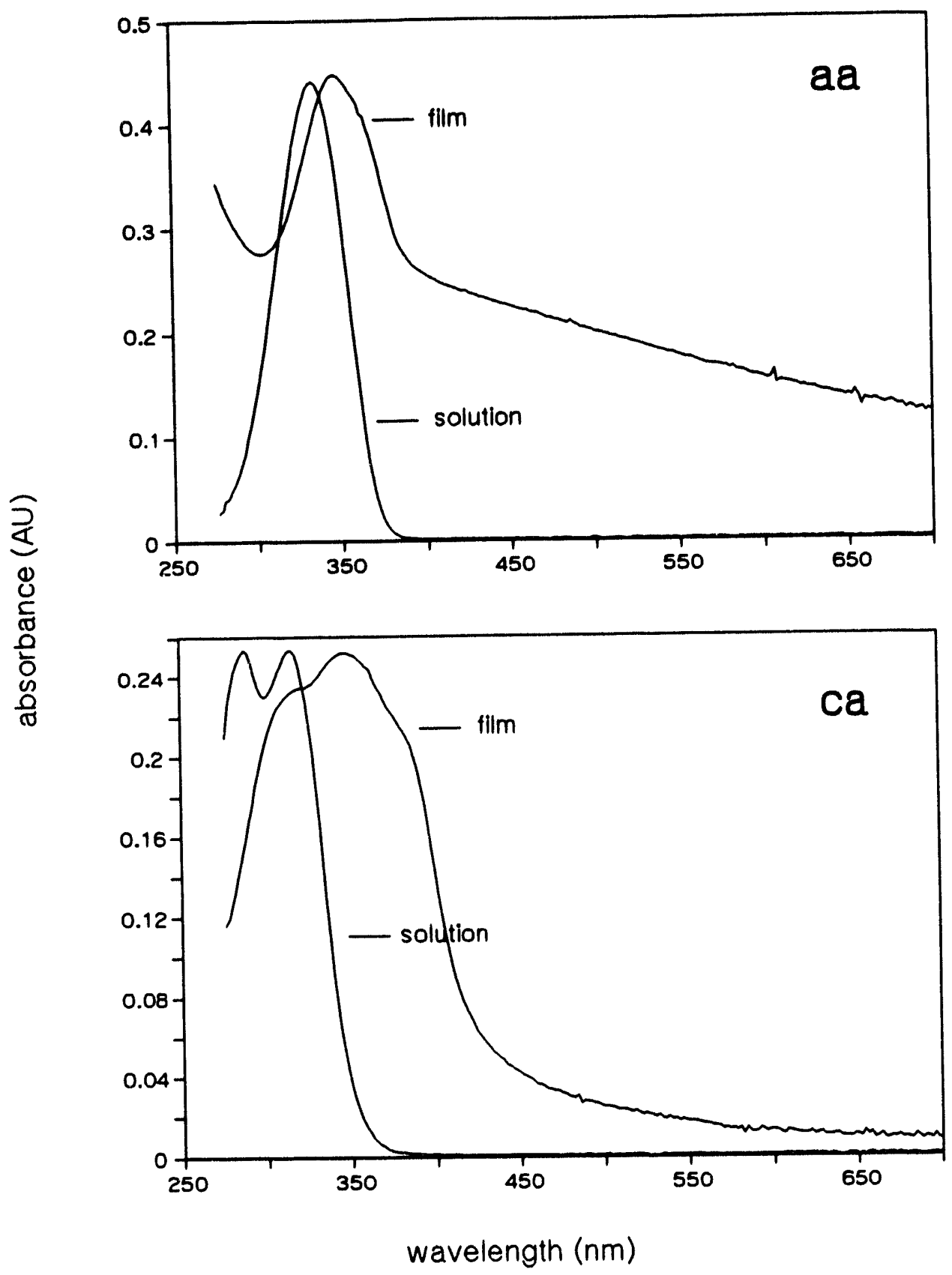

Figure 2. Absorbance spectra of UV matrices. aa $=$ anthranilic acid (solution $=$ $160 \mu \underline{\mathrm{M}}$, film $=133 \mathrm{nmol} / \mathrm{cm}^{2}$ ); ca $=$ caffeic acid (solution $=32 \mu \underline{\mathrm{M}}$, film $=20 \mathrm{nmol} / \mathrm{cm}^{2}$ ) 

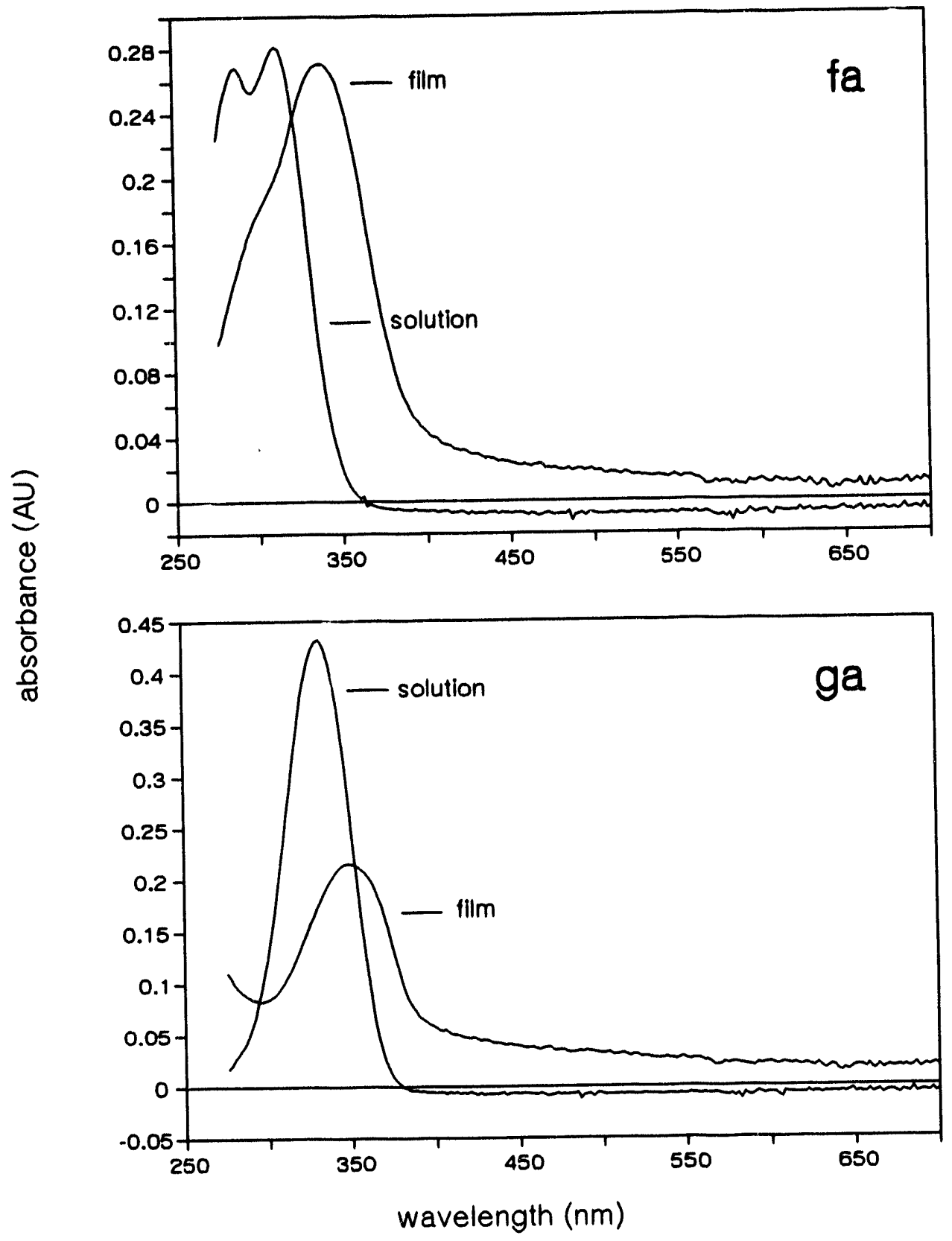

Figure 2. (continued) $\mathrm{fa}=$ ferulic acid (solution $=32 \mu \underline{\mathrm{M}}$, film $=24 \mathrm{nmol} / \mathrm{cm}^{2}$ ); $\mathrm{ga}=$ gentisic acid (solution $=160 \mu \underline{\mathrm{M}}$, film $=24 \mathrm{nmol} / \mathrm{cm}^{2}$ ) 


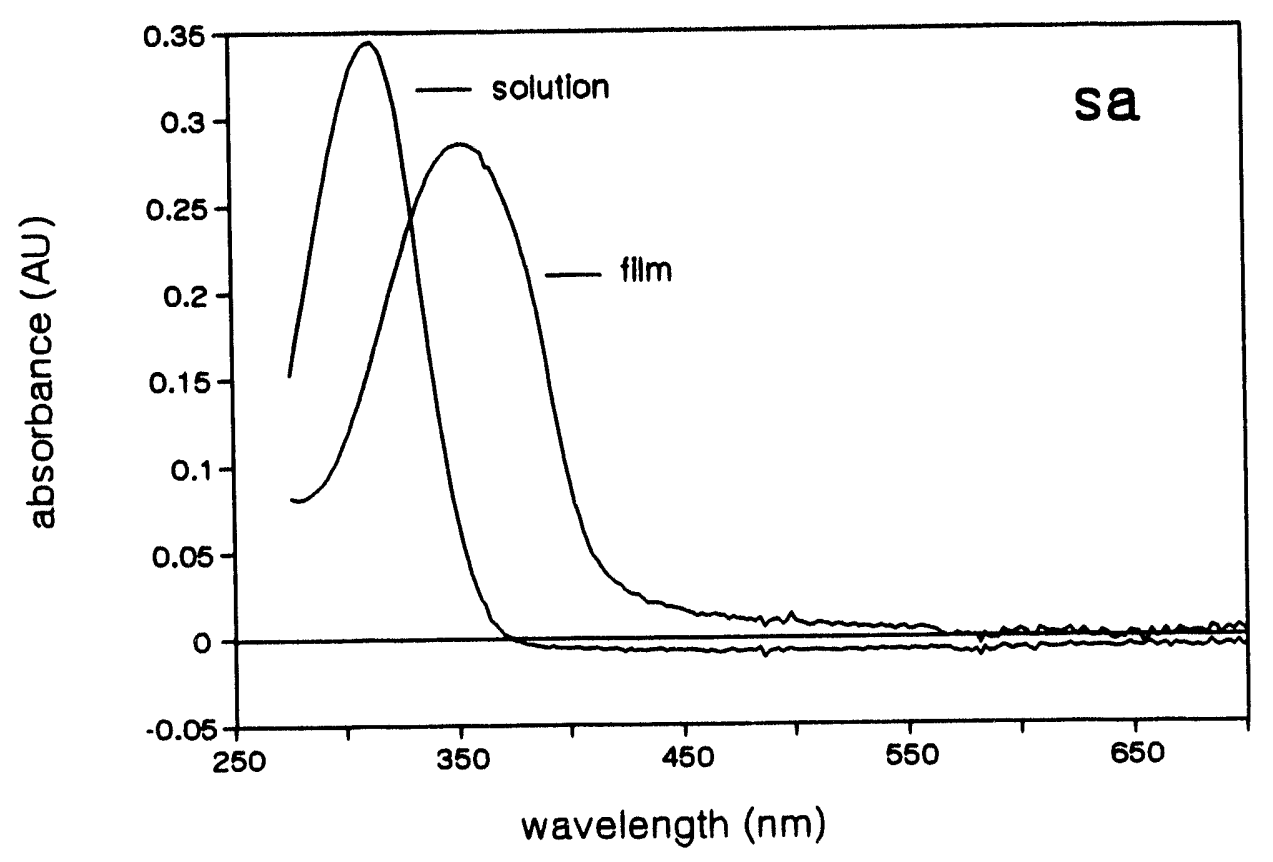

Figure 2. (continued) $\mathrm{sa}=$ sinapinic acid (solution $=32 \mu \underline{\mathrm{M}}$, film $=27$ $\mathrm{nmol} / \mathrm{cm}^{2}$ )

absorbance plot for ferulic acid is shown in Figure 3. Absorbance was determined for each film thickness at five different positions on the film. The error bars indicate \pm 1 standard deviation and give a sense of film uniformity.

The molar absorptivities $(\mathrm{L} / \mathrm{mol} \cdot \mathrm{cm})$ for dye films on quartz plates are shown in Table III. These values were derived from the observed absorbance over a calibrated area of uniform films of known concentration. These follow the general trend of 
Table II. Molar absorptivities for matrix solutions and films at $338 \mathrm{~nm}$

\begin{tabular}{lll}
\hline matrix & $\begin{array}{l}\text { film } \epsilon \\
(\mathrm{L} / \mathrm{mol} \cdot \mathrm{cm})\end{array}$ & $\begin{array}{l}\text { solution } \epsilon \\
(\mathrm{L} / \mathrm{mol} \cdot \mathrm{cm})\end{array}$ \\
\hline anthranilic acid & 2700 & 3300 \\
caffeic acid & 3000 & 8400 \\
ferulic acid & 9500 & 8000 \\
gentisic acid & 2800 & 3100 \\
sinapinic acid & 9700 & 11000 \\
\hline
\end{tabular}

Table III. Molar absorptivities for dye films used in $\mathrm{L} / \mathrm{mol} \cdot \mathrm{cm}$

\begin{tabular}{lrrr}
\hline matrix & $\epsilon_{338}$ & $\epsilon_{354}$ & $\epsilon_{488}$ \\
\hline rhodamine 6G & 1600 & 2400 & 5000 \\
disodium fluorescein & 1400 & 810 & 6500 \\
coumarin 334 & 600 & 690 & 2200 \\
coumarin 6 & 120 & 370 & 2500 \\
\hline
\end{tabular}

solution measurements, but do not match the latter exactly because of the different environments. These values are however all substantially lower than the molar absorptivities of the matrix materials. This, plus the large matrix to analyte ratio, imply that coupling of the laser beam is primarily via the matrix material. 


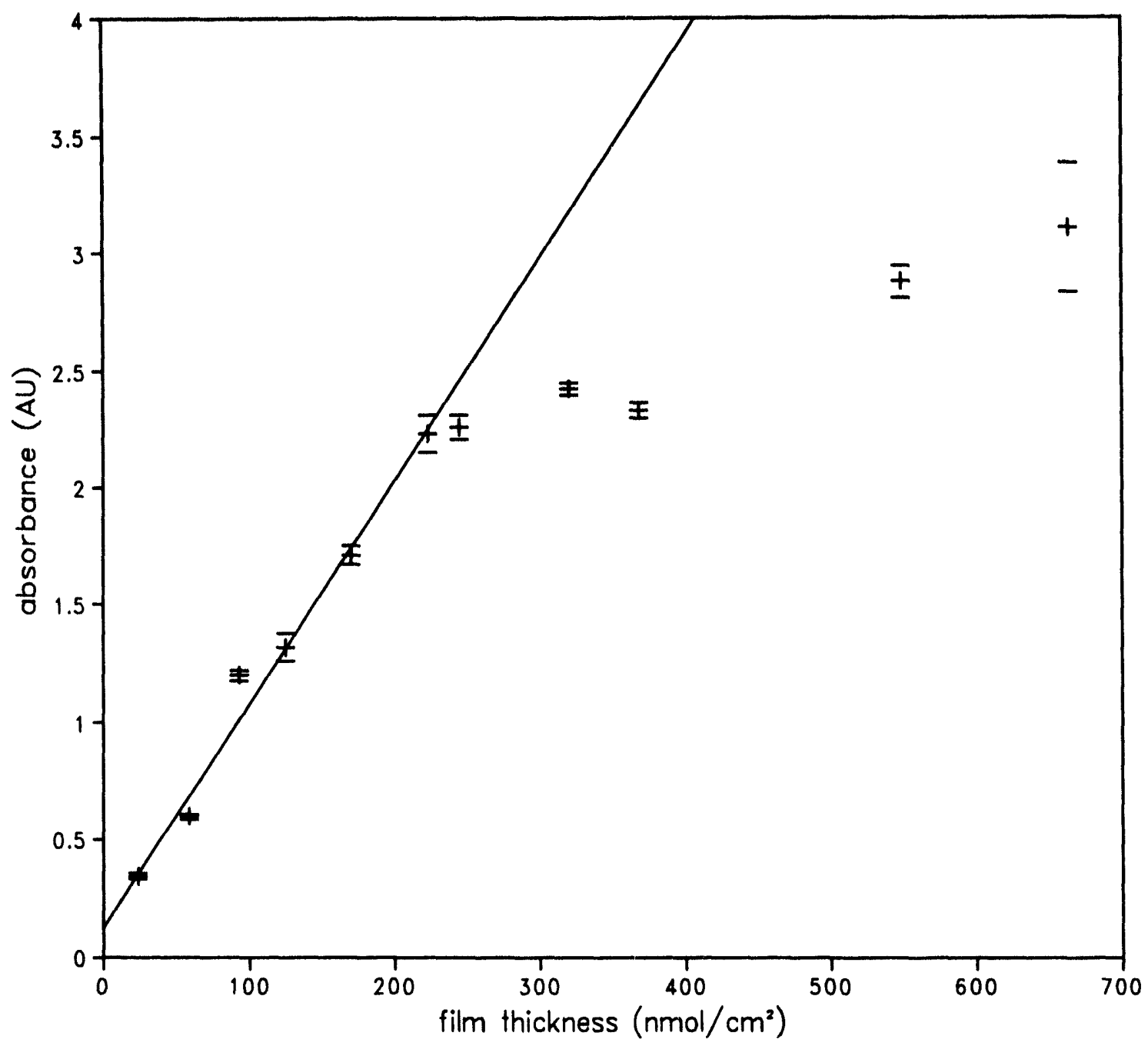

Figure 3. Absorptivity determination for ferulic acid 


\section{CHAPTER 4. QUANTITATION OF REMOVAL}

In typical UV-MALD work, the small amount of material removed per laser pulse (typically 10 attomol or less ${ }^{74}$ ) is difficult to quantitate. ${ }^{41}$ The greater amount of material removed in IR-MALD has made direct microscopic estimation of sample removal possible. ${ }^{74} \mathrm{~A}$ knowledge of the amount of material removed with each laser pulse may prove to be useful. In this chapter approaches to such quantitation of the common UV matrices are presented.

Frontside and backside desorption gave distinctly different results. When the desorption laser irradiance was less than $\sim 1 \mathrm{MW} / \mathrm{cm}^{2}$, desorption was incomplete or absent regardless of ge Jmetry. At higher irradiances (i.e., $>1.5 \mathrm{MW} / \mathrm{cm}^{2}$ ) backside desorption was virtually complete with no observable film residue remaining. Frontside desorption removed considerably less sample. Depending on film thickness, 3 to 30 shots were required tor complete sample removal. For backside desorption the cleaned sample area was found to be a function of laser pulse energy. This can be attributed to the threshold nature of the desorption process. As pulse energy increases, an increasing portion of the beam profile is above threshold fluence. Since the films are of finite thickness, once the desorption threshold is exceeded, no additional material is removed from the sample surface with increased irradiance.

\section{Areal Method}

The simplest approach is most desirable -- especially if it works. For this method the film thickness was determined by straight-forward calculation from the 
amount deposited divided by the area covered. Of course this approach depends on the assumption of uniform sample distribution. The area cleaned was measured with a microscope fitted with a reticle. Quantitation of amount desorbed was then taken to be the total amount deposited factored by the fraction of cleaned area to total area. Since frontside desorption did not clean the slides, areal quantitation was not possible for that geometry.

\section{Acoustic Study}

Previous work in this lab has demonstrated that laser ablation generates an acoustic signal which is directly proportional to the amount of material removed..$^{73,76}$ This effect has been observed in other laboratories as well. ${ }^{77}$ This section describes attempts to extend this relationship to the MALD process.

The acoustic experiment was set up as depicted in Figure 4. The acoustic transducer used was an Archer wide-range response replacement condenser microphone element (Radio Shack, no. 270-092B). The listed response was essentially flat over the frequency range from $20 \mathrm{~Hz}$ to approximately $16 \mathrm{kHz}$. The microphone was positioned $\sim 36 \mathrm{~mm}$ from the desorption site along the sample surface normal.

A typical acoustic waveform is shown in Figure 5. The arrival of the first major deflection can be seen at $104 \mu \mathrm{s}$. The sample to microphone distance divided by the transit time yields a velocity of $346 \mathrm{~m} / \mathrm{s}$. This value is typical of the speed of sound in air and, ignoring any super-sonic component, ${ }^{78}$ serves as an independent check on the sample to microphone distance. 


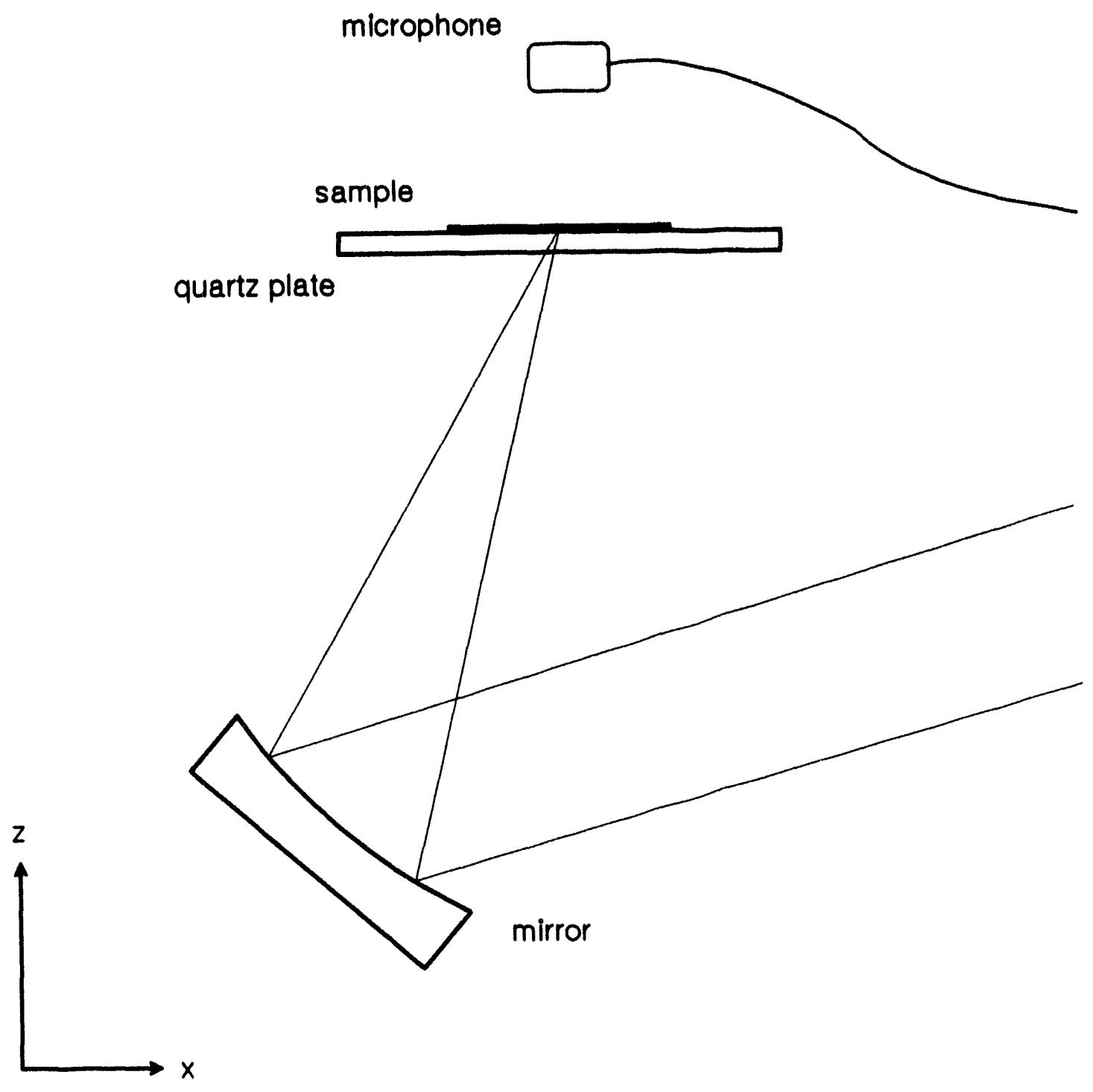

Figure 4. Block diagram of acoustic experimental setup 


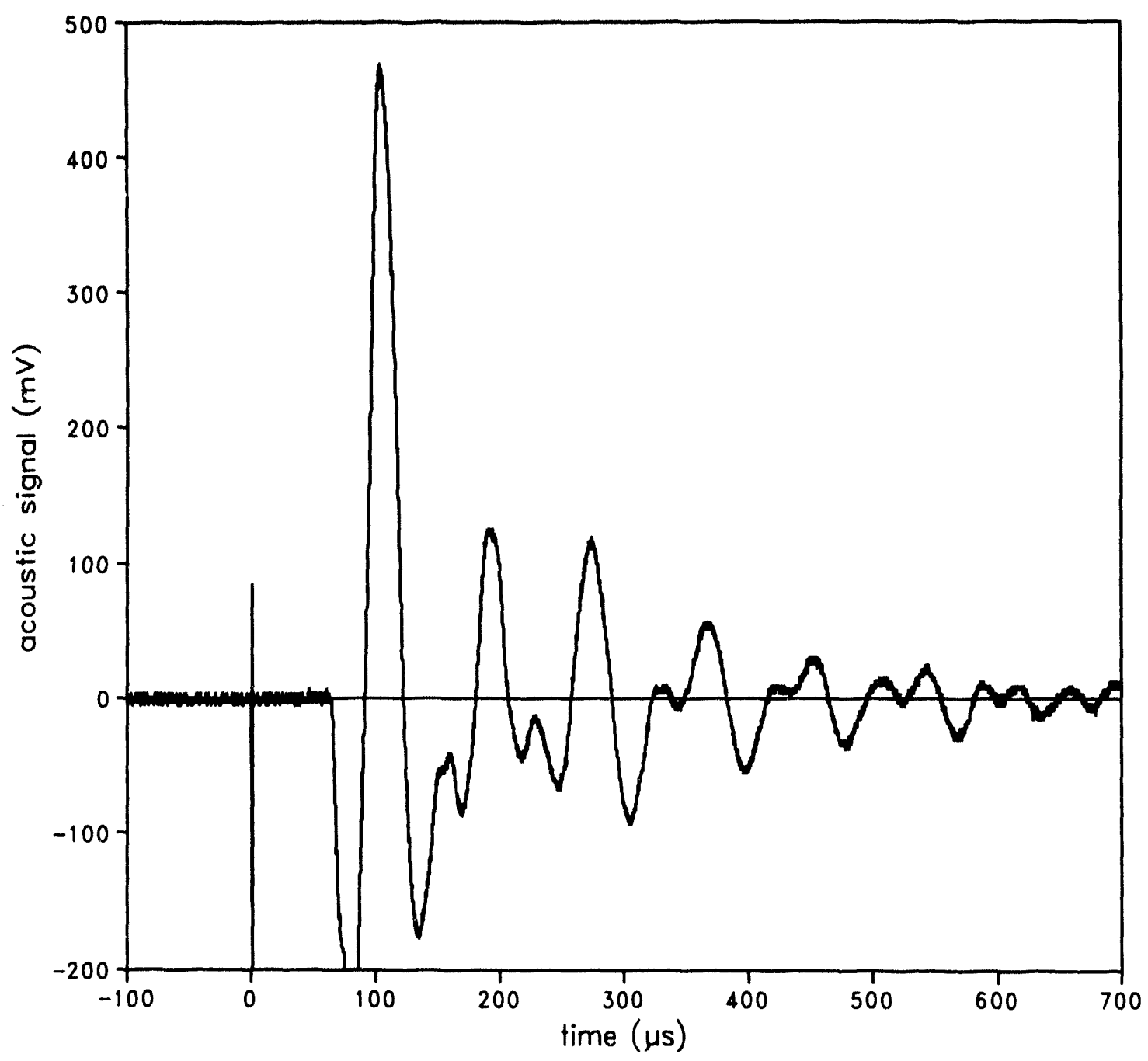

Figure 5. Typical acoustic signal for frontside desorption of ferulic acid; film thickness $16 \mathrm{nmol} / \mathrm{mm}^{2}$, microphone to sample distance $\sim 36 \mathrm{~mm}$ 
Correlation of acoustic signal to amount removed was not possible. There are several causes. For frontside desorption, sample removal was generally incomplete. This made the areal estimation method impossible. For backside desorption, sample removal was virtually complete. However, no definite correlation of acoustic signal to area of sample removal was found. Acoustic signal arises as a result of compression of the surrounding atmosphere by the rapidly expanding plume. It is suggested that backside desorption vaporizes only a portion of the sample closest to the quartz plate. The remainder of the sample is left with no physical attachment to the quartz, but driven away from the quartz surface by the expansion of the intervening material. Although sample removal was complete, it is likely that only the vaporized portion contributed to the acoustic signal. If this picture is true, it can be further hypothesized that the non-vaporized portion of the film could have a quenching effect on the expansion of the vapor and the resulting acoustic signal.

In Figure 6 frontside acoustic signal is plotted as a function of film thickness. A general trend is immediately obvious. The acoustic signal is rather linear with film thickness up to a certain point. Results from the backside experiment are presented in Figure 7. It is interesting to note that the backside and frontside results show essentially the same trend. Although the general appearance is much alike, there are some differences. In the frontside experiment the plot is basically flat above a certain film thickness. The backside experiment levels off at nearly the same film thickness, but shows considerably more variation at greater film thickness. This greater variability tends to support the notion that film material beyond the vaporized region 


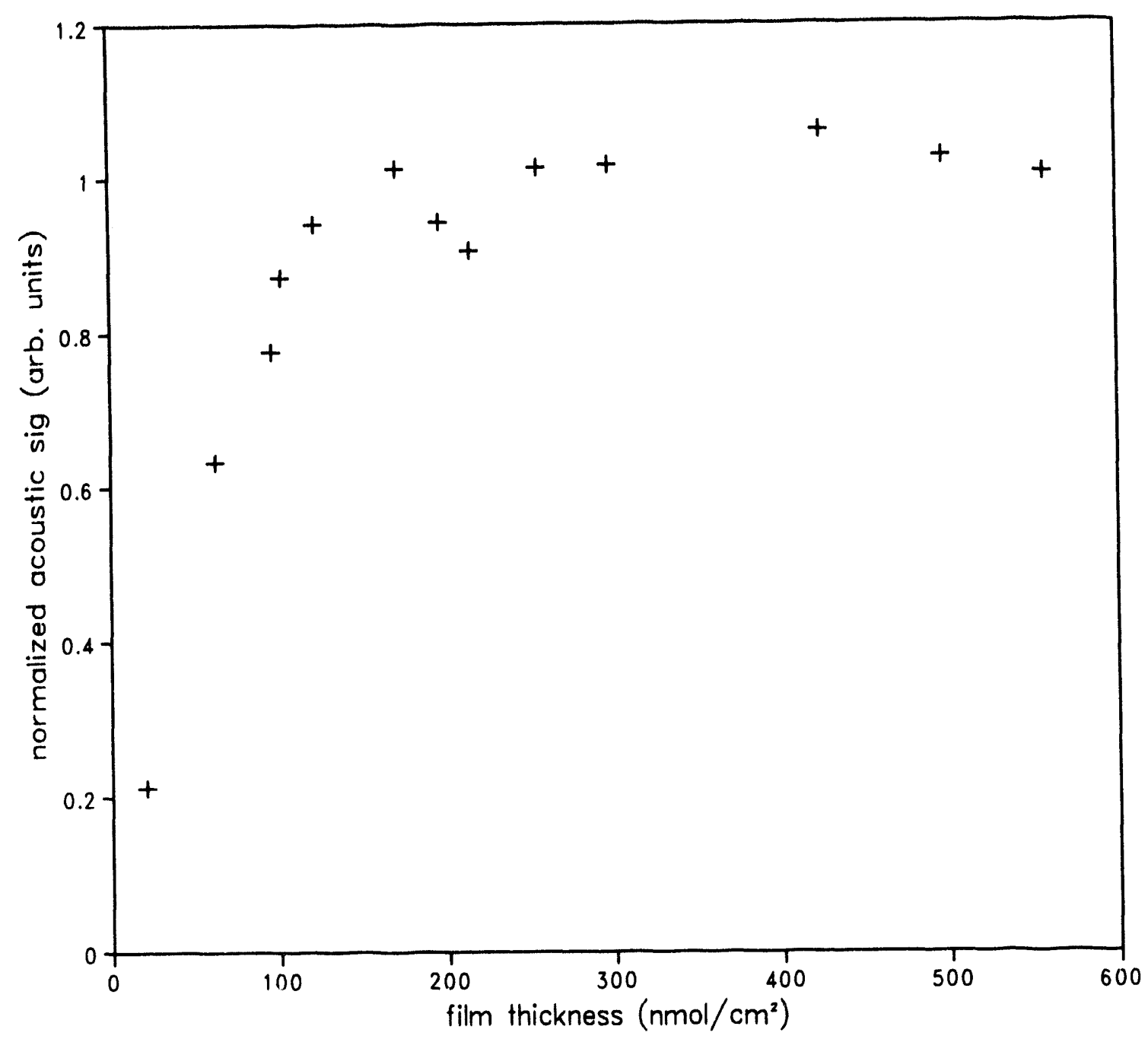

Figure 6. Frontside desorption of ferulic acid films 


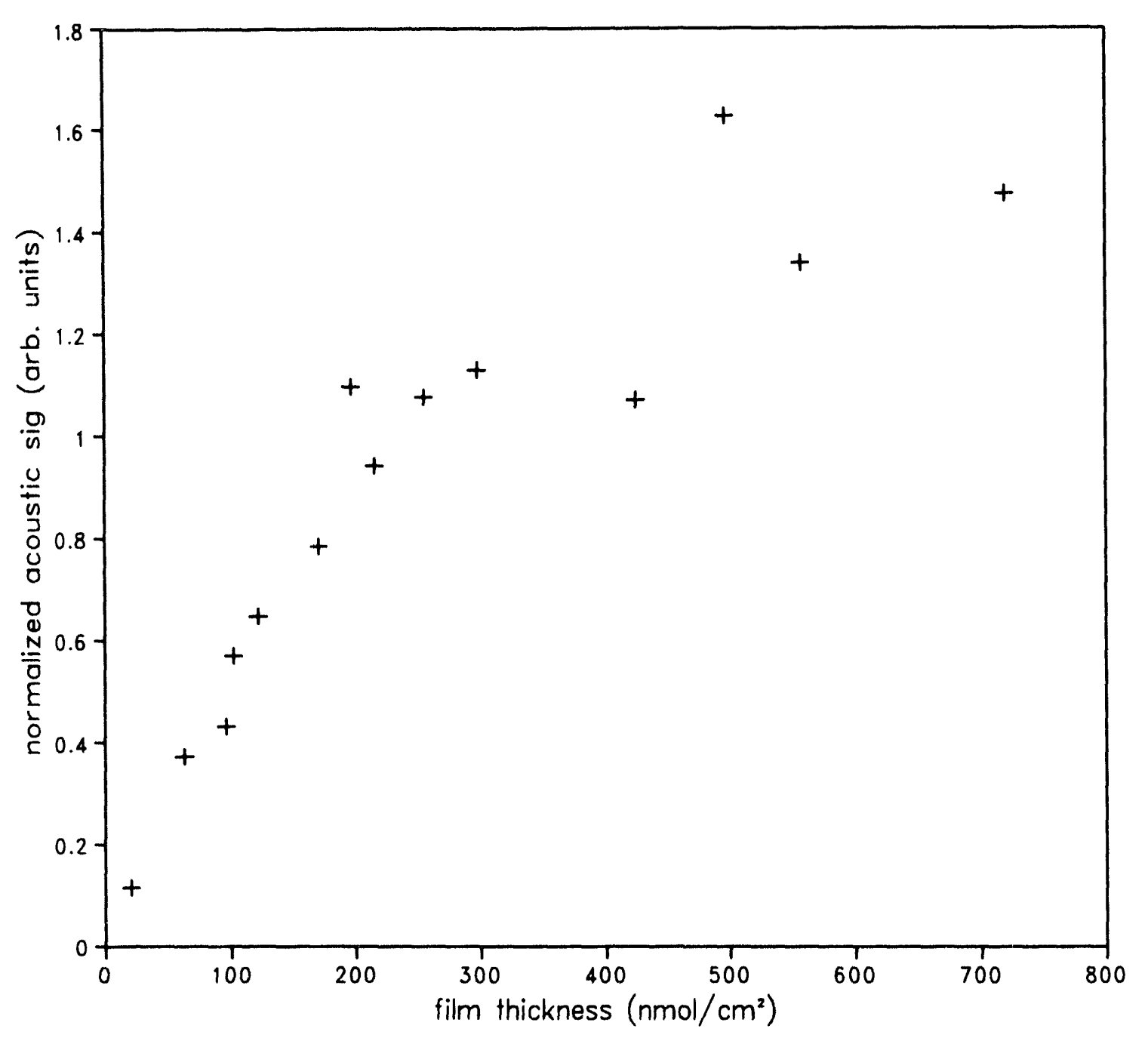

Figure 7. Backside desorption of ferulic acid films 
impedes the development of the acoustic signal. The same trend manifests itself in the caffeic acid work and is shown in Figure 8. It should be pointed out that the acoustic signal is also a function of the energy delivered by the vaporization laser. The data in Figure 6 and Figure 7 have been normalized to compensate for variations in that energy. More will be said about this energy dependence in Chapter 5.

\section{Quartz Crystal Microbalance}

A review of the quartz crystal microbalance $(\mathrm{QCM})$ has recently been published. ${ }^{79}$ This review is directed primarily at electrochemical applications, but contains an excellent introduction to the general theory. The QCM is based upon the reverse piezoelectric effect. In this effect, an AT cut quartz crystal, in conjunction with appropriate circuitry, oscillates at a stable frequency which is proportional to the mass of the resonator. Through use of the Sauerbray equation ${ }^{80}$ and a suitable frequency counting device, changes in crystal mass can be determined from the measured frequency changes. This relationship can be extended to encompass mass changes in films of material in intimate contact with the crystal. This use of the Sauerbray equation involves several assumptions. Among these are that the film have the same density and acoustic impedance as quartz, and that there be no slippage between the crystal and its attached film. High mass loadings can decrease QCM sensitivity. Buttry and Ward state that QCM measurements are typically considered accurate if film mass is less than $2 \%$ of the crystal mass. ${ }^{79}$ The thin films studied here are decidedly less than that level and minimize concern about the assumptions. 


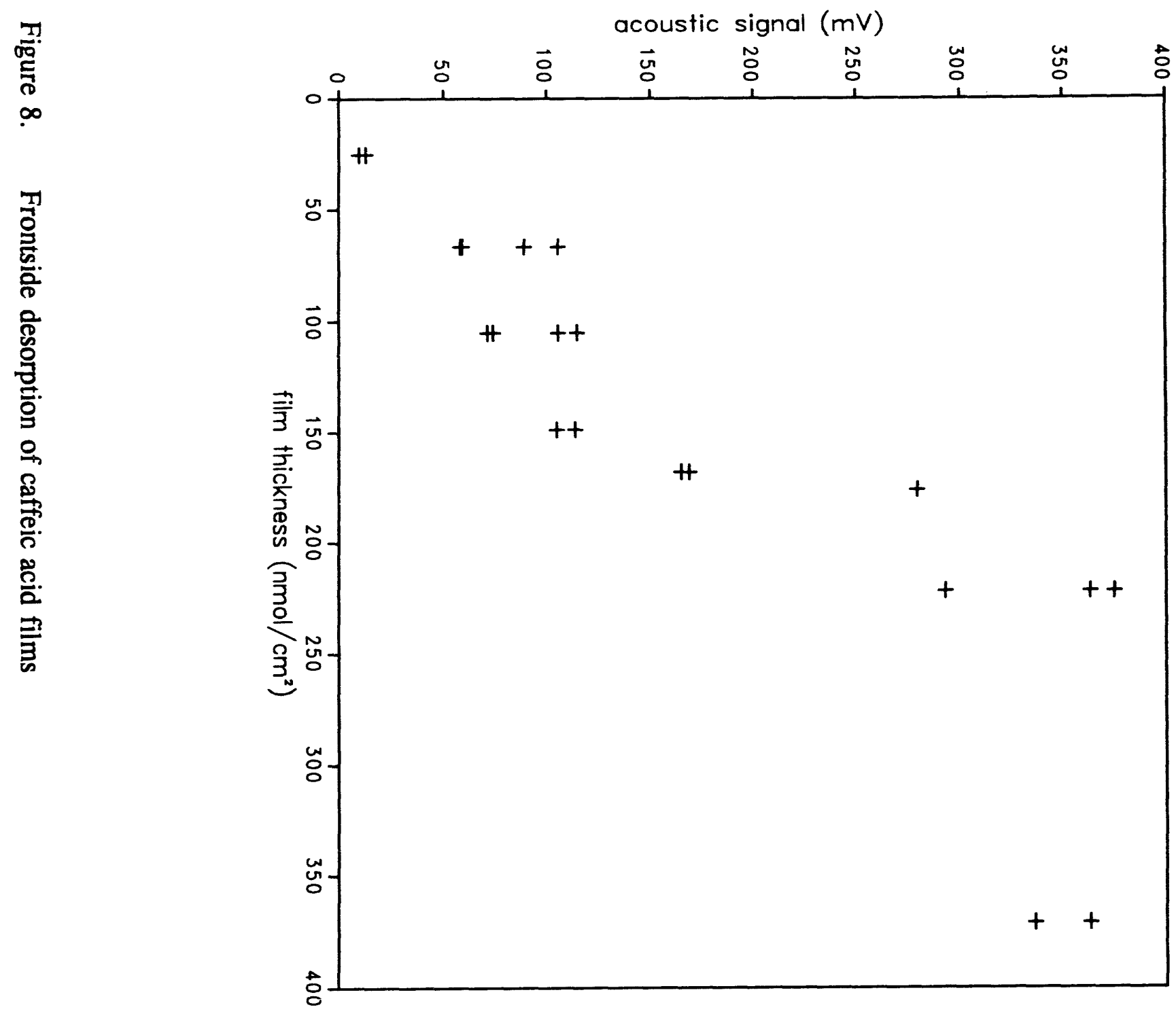


A further complication of the ideal Sauerbray case is that the QCM response is not uniform across the entire crystal surface. The QCM typically exhibits a Gaussian type response with the center having the highest degree of sensitivity. ${ }^{81,82}$ For that reason, desorption was performed at the electrode center.

The goal of this work was to use the mass sensitivity of the QCM to correlate acoustic signal to independently measured mass shanges associated with MALD events.

\section{Experimental}

For the QCM work, the quartz sample plates were replaced with the QCM crystal oscillator. The oscillator consisted of a $6 \mathrm{MHz}$ AT cut quartz crystal from Maxtek, Inc (part no. 1032000) connected with an oscillator controller chip (Texas Instruments \#74LS321) as shown in Figure 9. The electrode configuration was a keyhole type design and had an active area of approximately $31 \mathrm{~mm}^{2}$. A photon counter in conjunction with an amplifier/discriminator (Ortec, models 9315 and 9302 respectively) was used to determine oscillation frequency. The desorption laser, focussed as outlined previously for frontside illumination, was directed at the center of the electrode active area. Because the electrode was not transparent to UV light, all QCM desorption was done in frontside geometry.

Results

The results of the QCM work were quite interesting. Figure 10 shows the stepwise increase in QCM frequency with successive laser pulses. Laser pulses occur at approximately every tenth QCM reading. Each step increase in QCM frequency 


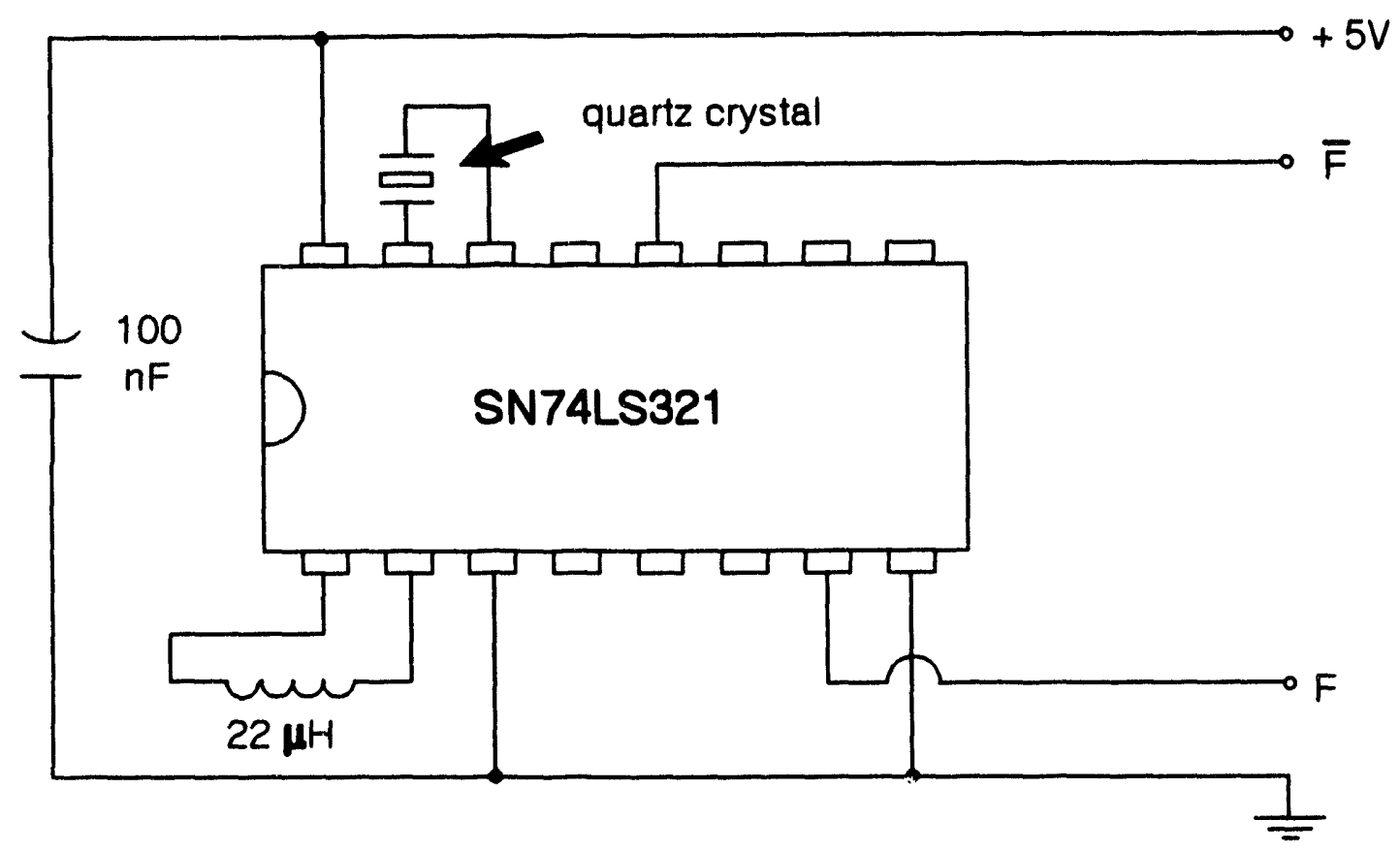

Figure 9 Schematic of QCM oscillator circuit; F = output to frequency counter 


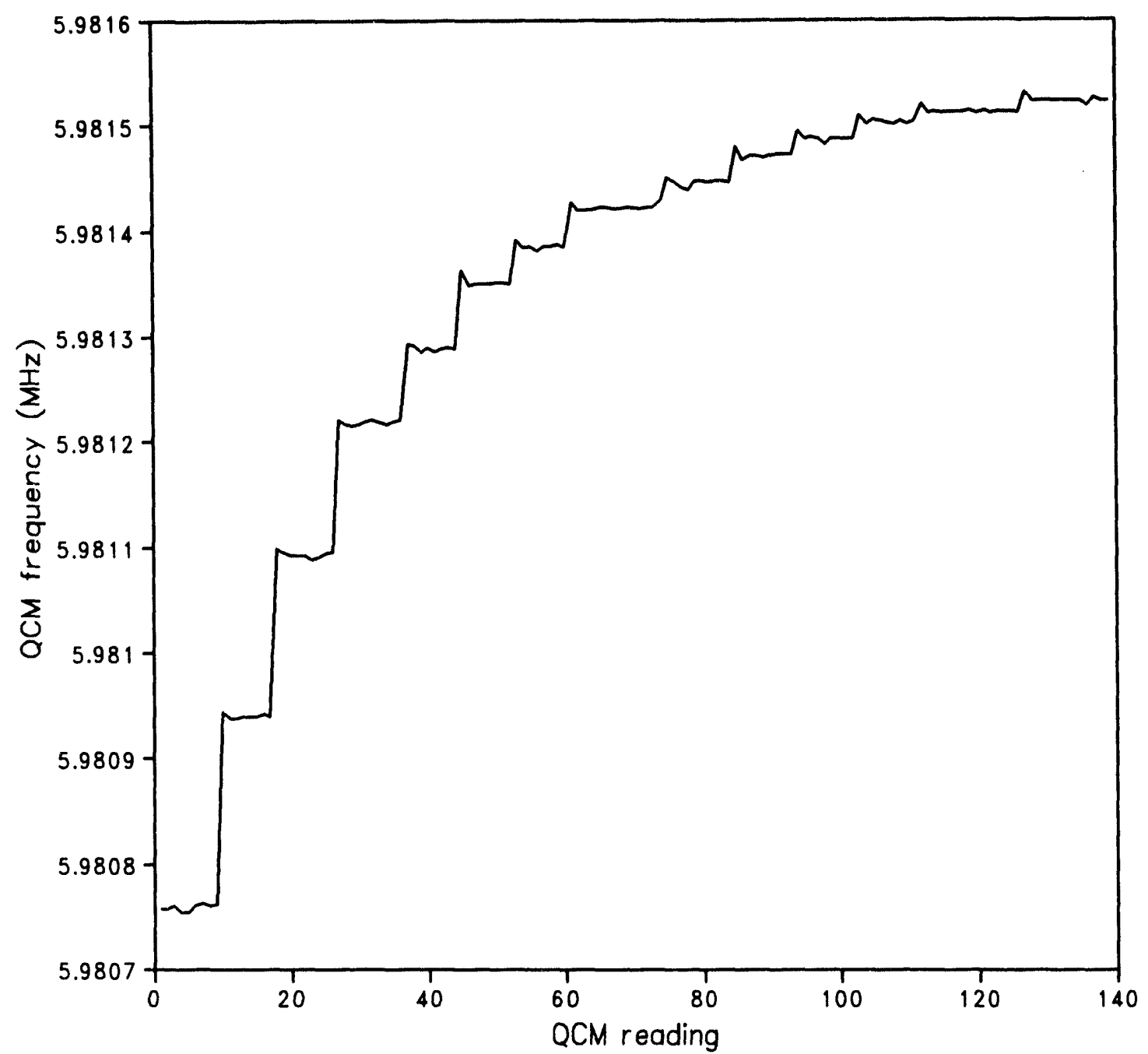

Figure 10. Frontside desorption of $700 \mathrm{nmol} / \mathrm{cm}^{2}$ ferulic acid film from a $\mathrm{QCM}$ 
represents loss of film material from a desorption event. Given the relationship between frequency change and mass change, it is apparent that the greatest removal of material comes with the first several pulses. As pulses continue, sample removal is completed, the frequency change approaches zero, and the QCM frequency approaches a steady state. After approximately a dozen desorption pulses, sample removal is essentially complete. The overall frequency change in this example is $764 \mathrm{~Hz}$. Assuming ideality of the Sauerbray equation, this corresponds to a mass removal of 1.5 $\mu \mathrm{g}$. The areal method of quantitation (discussed in a previous section) indicates a mass removal of $2.2 \mu \mathrm{g}$. There is a significant discrepancy between these values, but when one considers all the assumptions involved in each calculation, the agreement is surprisingly good. The fact that the QCM value is the lower of the two suggests the difference may be attributed to the decreased sensitivity of the QCM for material offcenter.

When the bare QCM was initially irradiated, a slight increase in oscillator frequency $(\sim 6 \mathrm{~Hz})$ over about 60 laser pulses was observed. Recently an ablation threshold of $0.6 \mathrm{~J} / \mathrm{cm}^{2}$ for gold from a QCM electrode has been demonstrated ${ }^{81}$. Based on typical fluences used in this work $\left(-0.1 \mathrm{~J} / \mathrm{cm}^{2}\right)$ it is unlikely that this frequency change is due to ablation of gold. In fact one run consisting of over 1800 laser pulses showed no change in QCM frequency for the last 1500 pulses. It is suggested that the initial frequency change may be due to removal of a surface oxide. Visual inspection of the QCM electrode after irradiation showed the desorption area to be noticeably shinier, supporting this suggestion. Furthermore, due to attenuation of the desorption 
pulse by the sample film, it is unlikely that much gold removal would occur until a significant portion of the sample had been removed. Compared to the overall frequency change of over $700 \mathrm{~Hz}$, this small contribution can reasonably be ignored.

Now let us consider the relationship of the QCM and acoustic signals.

Figure 11 shows acoustic signal plotted as a function of change in QCM frequency. This plot is linear with a correlation coefficient of 0.997 . The non-zero intercept is possibly due to a background acoustic signal generated by the rapid thermal expansion of the bare gold electrode. Leung and Tam have observed an acoustic signal at subthreshold laser irradiances. ${ }^{78}$ They refer to this as a thermal piston signal. Heating effects are also the likely cause cause of spiking observed at the leading edge of each step in Figure 12. In Figure 11 each initial QCM reading after a laser pulse (corresponding to the spikes) has been dropped.

Although quantitation of MALD by acoustic signal could not be definitely confirmed by an independent method, the linear relationship between acoustic signal and both film thickness and QCM response suggest that the idea is worth pursuing. 


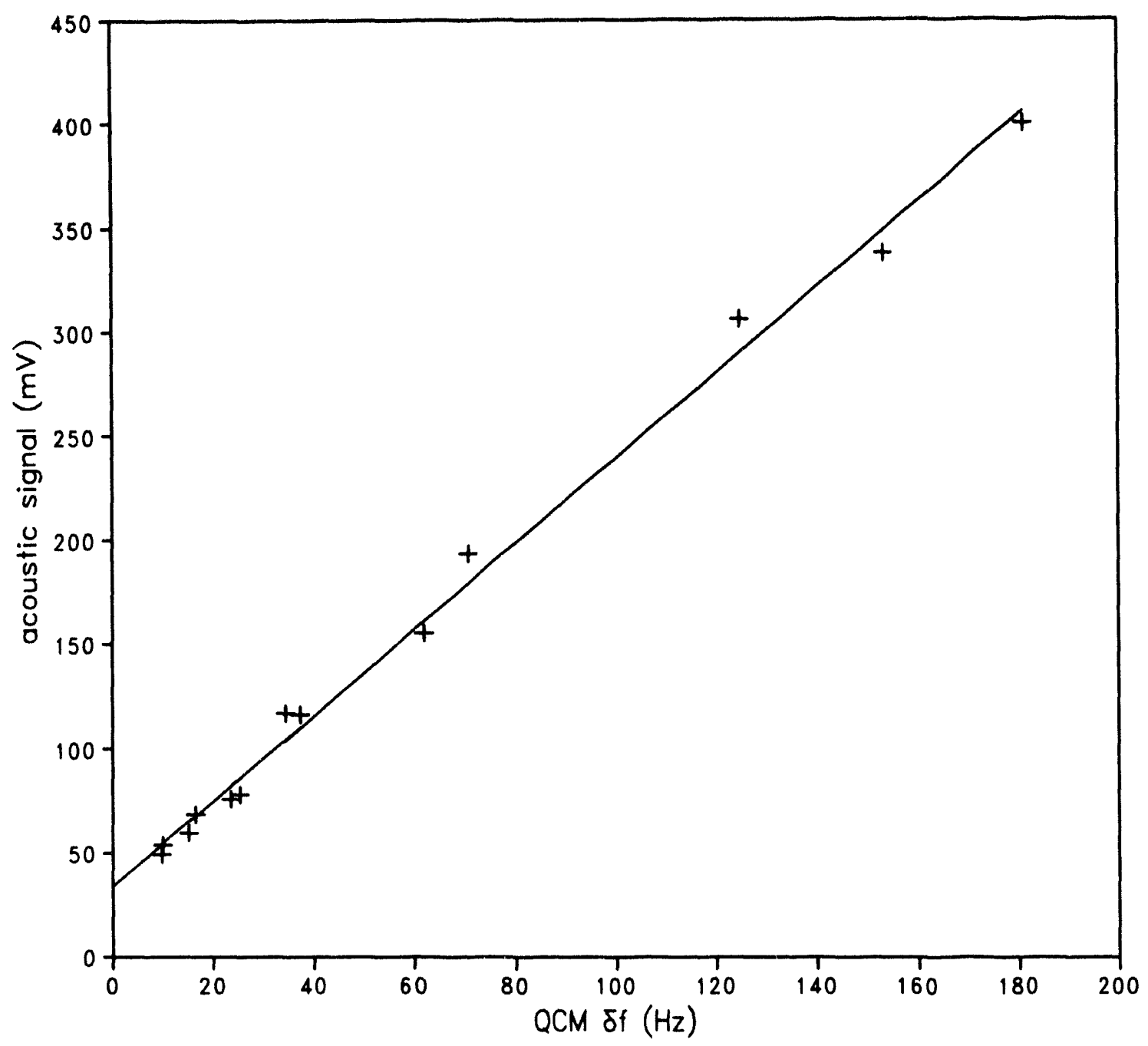

Figure 11. Frontside desorption of $700 \mathrm{nmol} / \mathrm{cm}^{2}$ ferulic acid film 


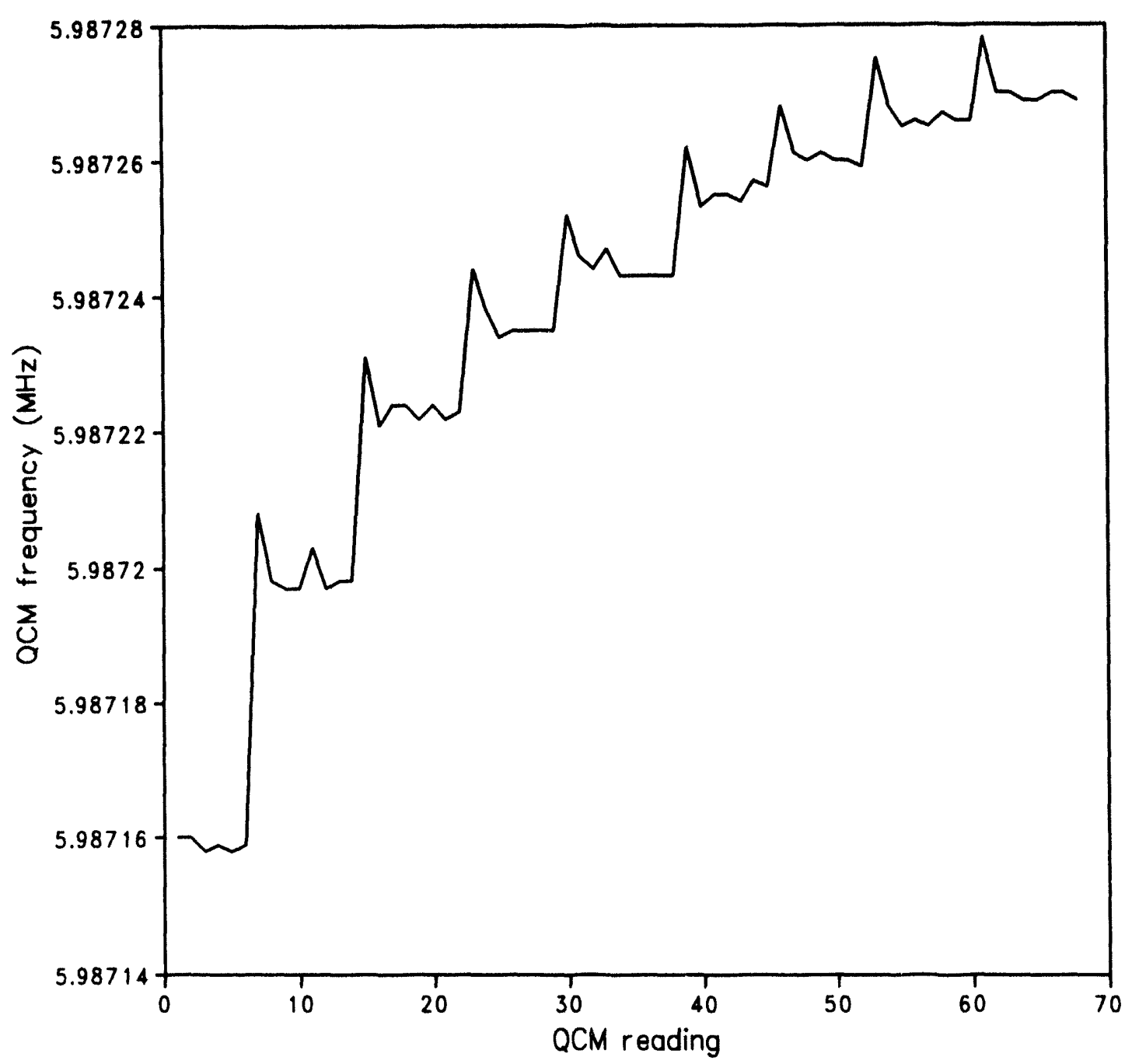

Figure 12. Raw data for frontside desorption of $700 \mathrm{nmol} / \mathrm{cm}^{2}$ ferulic acid from a quartz crystal microbalance 


\section{CHAPTER 5. DESORPTION THRESHOLDS}

As previously mentioned, there was a decided dependence of acoustic signal upon nitrogen laser energy. In fact this relationship was found to be extremely linear. The linear fits gave decidedly non-zero $x$-axis intercepts which tended to be remarkably independent of film thickness. Table IV shows values determined for a range of ferulic

Table IV. Acoustic thresholds for desorption of ferulic acid

\begin{tabular}{lll}
\hline $\begin{array}{l}\text { film thickness } \\
\left(\mathrm{nmol} / \mathrm{cm}^{2}\right)\end{array}$ & $\begin{array}{l}\text { frontside threshold } \\
(\mathrm{mJ})\end{array}$ & $\begin{array}{l}\text { backside threshold } \\
(\mathrm{mJ})\end{array}$ \\
\hline 720 & 0.95 & 1.45 \\
557 & 1.18 & 1.61 \\
497 & 1.29 & 1.72 \\
424 & 1.26 & 1.42 \\
298 & 1.26 & 1.34 \\
255 & 1.34 & 1.25 \\
215 & 1.41 & 0.96 \\
197 & 1.24 & 1.08 \\
171 & 1.15 & 1.18 \\
122 & 1.17 & 1.18 \\
103 & 1.17 & 1.44 \\
96 & 1.17 & 1.20 \\
63 & 1.21 & 1.38 \\
21 & 1.05 & 1.22 \\
mean & 1.20 & 1.32 \\
standard deviation & 0.11 & 0.20 \\
\hline
\end{tabular}


acid film thicknesses. It is suggested that these intercepts, corresponding to a minimum pulse energy, represent a threshold value for a desorption event. Below this threshold there is insufficient energy to initiate a desorption event.

Backside desorption gives about $10 \%$ higher thresholds than frontside. Most of this difference is contributed from the thicker films. It was mentioned previously that a qualitative explanation for complete sample removal in backside geometry might be vaporization of sample closest to the quartz which drives the remaining unvaporized sample away. If this explanation is correct, the energy required to remove the unvaporized solid material could manifest itself as an increase in observed threshold.

At low laser irradiances, the signal showed a tendency (see Figure 13) to deviate positively. This is attributed to non-uniformity of the nitrogen laser beam profile. This unevenness creates hot spots where the local irradiance is significantly greater than the average irradiance. The result is that localized areas exceed the vaporization threshold and give rise to an acoustic signal. For this reason all laser pulses below the calculated thresholds were omitted from the regressions. With the omission of data points below the calculated threshold value, typical linear fits had correlation coefficients of 0.99 .

A wide range of values for ion production thresholds have been reported, ${ }^{30}$ but the most recent work has tended to values in the range of 1 to $10 \mathrm{~W} / \mathrm{cm}^{2}$. Specifically, the Hillenkamp group has reported a threshold for ion production using gentisic acid of $3 \times 10^{6} \mathrm{~W} / \mathrm{cm}^{2} .{ }^{41}$ Likewise, Ens and coworkers ${ }^{21}$ have reported a threshold value of 2 $\times 10^{6} \mathrm{~W} / \mathrm{cm}^{2}$ for sinapinic acid. These values agree remarkably well with our measurements for acoustic thresholds. Threshold values for all matrices studied are 


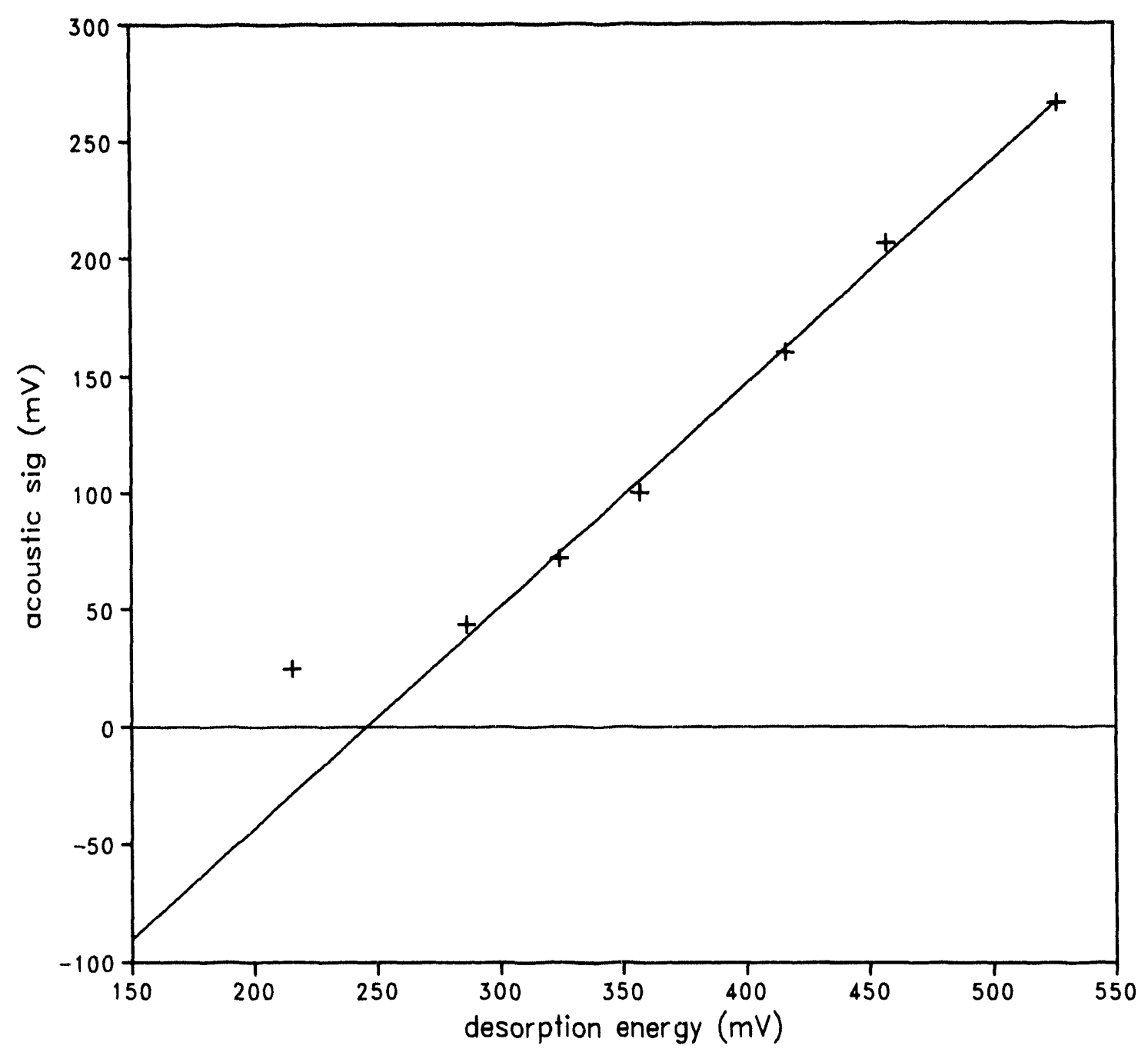

Figure 13. Frontside desorption of $557 \mathrm{nmol} / \mathrm{cm}^{2}$ ferulic acid 
Table V. Desorption thresholds determined for UV matrices

\begin{tabular}{llll}
\hline matrix & $\begin{array}{l}\text { molecular } \\
\text { weight } \\
(\mathrm{g} / \mathrm{mol})\end{array}$ & $\begin{array}{l}\text { desorption } \\
\text { threshold } \\
\left(\mathrm{MW} / \mathrm{cm}^{2}\right)\end{array}$ & film $\epsilon$ \\
$(\mathrm{L} / \mathrm{mol} \cdot \mathrm{cm})$
\end{tabular}

presented in Table V. If one ignores anthranilic acid, a correspondence between molar absorptivity and desorption threshold is seen. Specifically, larger molar absorptivity corresponds to a lower threshold. This seems reasonable. The effect of increasing $\epsilon$ would be to lower the irradiance required to reach some specific threhold energy value. The presence of an amine functional group exclusively on anthranilic acid could cause increased volatility and explain its different behavior.

The conclusion is that once enough energy has been supplied to the irradiated sample to initiate desorption, ionization also takes place. Ens has reported ion yield scaling by a high power (4th to 6 th) of desorption laser irradiance. ${ }^{21}$ The fact that our relationship is linear above threshold indicates that ion production may be occurring by mechanisms other than direct photoionization. It should be noted that our use of laser irradiance as the threshold parameter is somewhat arbitrary. Beavis has suggested ${ }^{83}$ that laser fluence is, in fact, the critical parameter. Work at Uppsala supports this 
view. ${ }^{84}$

The acoustic/energy relationship depicted in Figure 13 also showed a small dependence on film thickness. In Figure 14 one can see that the acoustic response increases with film thickness. This relationship was a general trend and did not hold for all cases. 


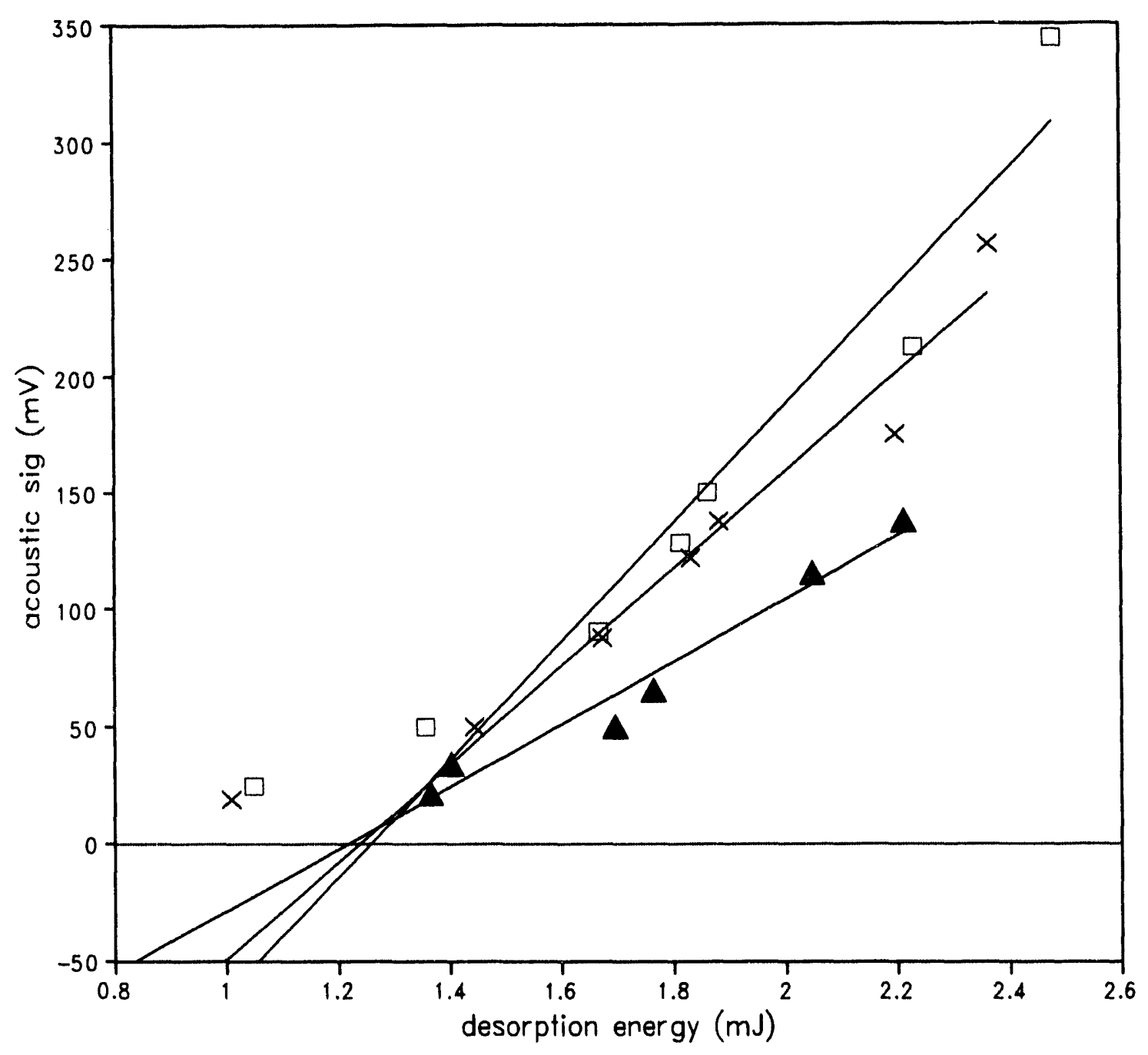

Figure 14. Acoustic response for frontside desorption of three ferulic acid films; 424 $\mathrm{nmol} / \mathrm{cm}^{2}(\square) ; 197 \mathrm{nmol} / \mathrm{cm}^{2}(\times) ; 63 \mathrm{nmol} / \mathrm{cm}^{2}$ (4) 


\section{CHAPTER 6. INTRODUCTION TO PLUME IMAGING}

The utility of the MALD technique for MS depends heavily on the formation of ions. The ionization mechanisms, however, are not well-known. There is belief that the dominant mechanism is proton transfer, ${ }^{57}$ but this is far from proven. As discussed in Chapter 2, there is evidence that a significant portion of desorbed analyte in MALD appears as neutral species. ${ }^{85}$ It was also pointed out that ionization efficiency falls off sharply outside a narrow range of laser irradiances. The immediate conclusion is that a secondary ionization scheme would help increase sensitivity substantially. Becker has suggested that post-ionization may be useful in cases where ions produced are few or are not characteristic of the sample. ${ }^{86}$ Increasing ionization efficiency could significantly increase detection limits and provide a broader range of applications. Finally, optimization of the desorption step itself can be most readily achieved if it is decoupled from the ionization step. ${ }^{87}$ Knowledge of the spatial and temporal dynamics of the laser desorbed plume would be most helpful in designing suitable secondary ionization schemes.

Attempts at mechanistic explanations for MALD have often turned to velocities of ejected ions as discussed in Chapter 2. In addition to aiding secondary ionization design, knowledge of plume dynamics might provide insight into the fundamental processes occurring in matrix-assisted laser desorption and thereby help to elucidate the operative desorption mechanism.

Over the years numerous attempts to image laser plumes have been made. Most 
have suffered from limitations. Some imaging techniques rely on combining data from the plume collected at different viewing sites. The drawback here is that the image is a composite of several plumes. Another approach uses a CCD detector to generate a complete image, but is limited to one image per plume due to the readout rate of the CCD. ${ }^{88}$

Optical spectroscopic probes based on lasers have been successfully employed for monitoring laser-generated plumes previously, providing spatial and temporal information on atoms, ${ }^{89}$ diatomics, ${ }^{90}$ and large molecules. ${ }^{11}$ These probes are based on absorption in the plume, ${ }^{11}$ reflection off the surface, ${ }^{91}$ and laser-enhanced ionization. ${ }^{92}$ The key to these imaging methods is the utilization of acousto-optic devices (AODs) to rapidly scan a probe laser beam through space. A recent review discusses the basics of AODs and their applications as tunable filters. ${ }^{93}$ The general theory is presented here for the interested reader.

\section{Acousto-optic Devices}

The acousto-optic devices used in our laboratory are based on diffraction of light and are sometimes referred to as Bragg cells. A schematic diagram of a generic AOD is presented in Figure 15. The Bragg cell itself consists of an acoustic medium which is transparent to the light wavelength of interest. A piezoelectric transducer (PZT) is placed in physical contact with the active medium. A radio frequency (RF) electromagnetic signal applied to the PZT creates an acoustic wave within the cell. At a constant frequency, the RF signal generates a standing acoustic wave. This wave is a 


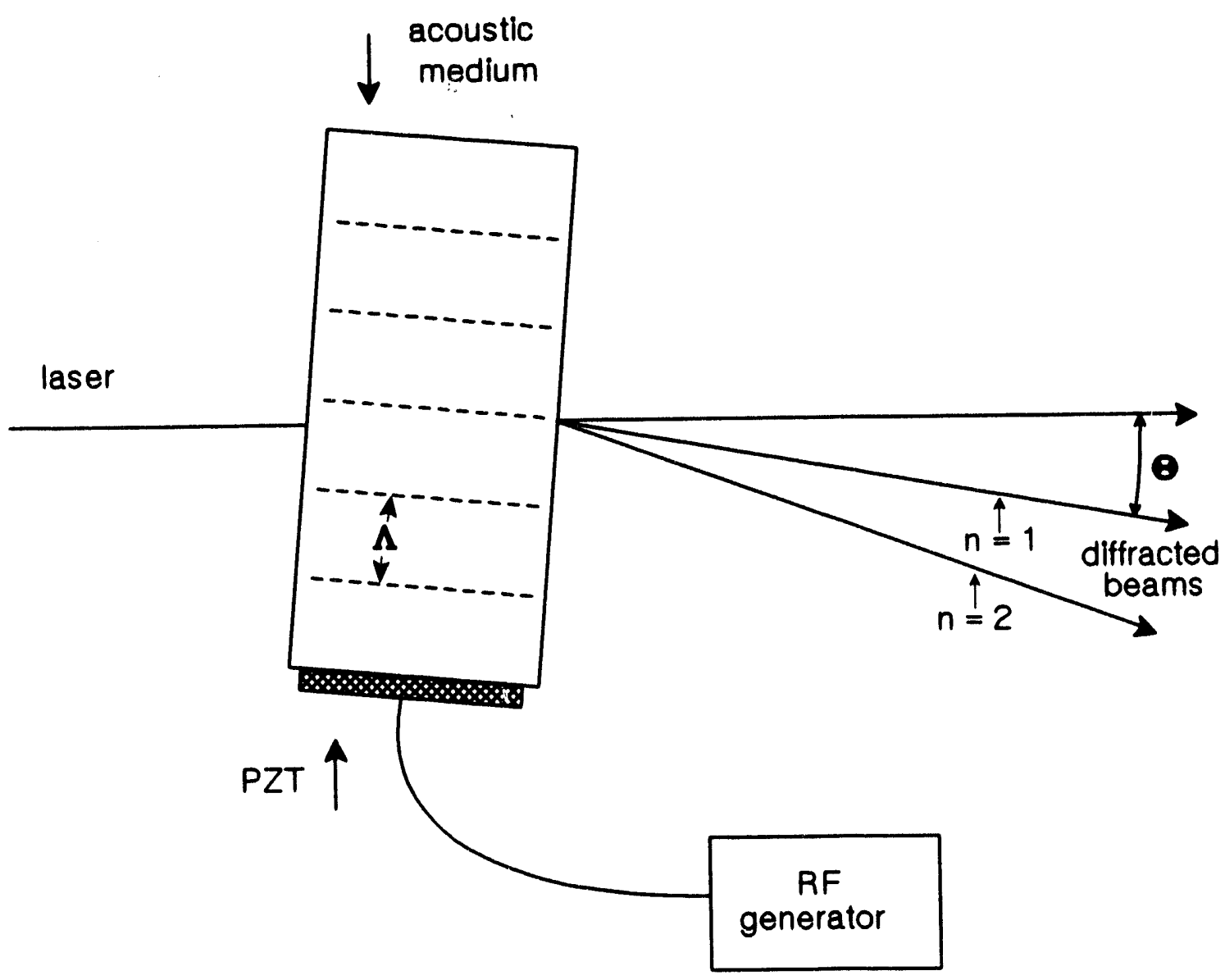

Figure 15. Schematic diagram of a typical acousto-optic device (see text for explanation) 
longitudinal wave consisting of alternating compressions and rarefactions of the cell material, which results in a sinusoidal variation in density. Because refractive index is a function of density, the end result is a periodic refractive index gradient. The periodic refractive index gradient acts as a transmission grating with groove spacing equal to the acoustic wavelength. Light incident upon the cell at the Bragg angle undergoes destructive and constructive interference with diffraction of light into the first and second order and beyond.

Equation (1) shows the pertinent relationship:

$$
\sin \theta=1 / 2(n \lambda / \Lambda)
$$

where

$$
\begin{aligned}
& \theta=\text { the diffraction angle, } \\
& n=\text { the order, } \\
& \lambda=\text { the incident light wavelength, } \\
& \Lambda \quad=\text { the acoustic wavelength. }
\end{aligned}
$$

By varying the applied RF signal about a center frequency, the diffraction angle can be changed. The result is that the laser beam can be scanned through space with a precise knowledge of its position at any point in time. Adding a second AOD orthogonal to the first and to the laser beam provides the capability of scanning the probe laser beam through two dimensions.

An important consideration in the use of an imaging system is the spatial resolution. The number of resolvable spots, $\mathbf{N}$, is given by: 


$$
N=1 / 4\left(D \pi \theta_{T} / \lambda\right)
$$

where

$$
\begin{aligned}
& \mathrm{D}=\text { the focused beam diameter, } \\
& \theta_{\mathrm{T}}=\text { the maximum deflection angle. }
\end{aligned}
$$

A caveat in the use of AODs is that one be aware of and compensate for the focussing properties they possess. The AOD operates in effect as a cylindrical lens ${ }^{94}$ with focal length, $f$, given by:

$$
\mathbf{f}=\mathbf{V T} / \boldsymbol{\theta}_{\mathrm{T}}
$$

$$
\text { where } \quad \begin{aligned}
\mathrm{V} & =\text { acoustic wave velocity, } \\
\mathrm{T} & =\text { total scan time. }
\end{aligned}
$$

At slower scan rates AOD lensing is not a significant effect. One can readily see that increasing scan rates leads to a decrease in $\mathrm{T}$ and hence, a decrease in focal length. For the scan rates used in our imaging system this lensing effect is significant and must be accounted for.

\section{Imaging Background}

The scanned probe beam can be used to excite spectrochemical processes such as absorbance, scattering, and fluorescence. The probe beam can excite these processes at a discrete location the size of which is limited only by the focus of the beam. Because of the finite resolution of the AOD, a tradeoff exists. For a given optical 
configuration each scan contains a fixed number of resolution elements. One can increase spatial resolution (i.e., spot size) only at the expense of scan length, or area interrogated.

The use of an AOD allows a probe laser beam to be scanned in one dimension. Addition of a second AOD oriented orthogonal to the first makes possible two dimensional scanning. The physical path the scanned probe beam follows is very similar to the manner in which an electron beam is scanned across a cathode ray tube to create a television image. One major difference is that the scan rate is about one hundred times more rapid in our experiment. To continue the television analogy, consider the probe beam, focused to a spot which is swept through space in two dimensions. The scanning creates a focal plane which is depicted in Figure 16a. Each complete sweep through this plaiıe is referred to as a frame. The probe beam starts the scan of a frame at the origin (the lower left corner) and su'eeps upward in the positive $z$ direction, away from the sample surface. Once it reaches the top of the plane, it is snapped back to the bottom to start a new vertical scan. At the same time it is being scanned horizontally in the $\mathrm{x}$ dimension, that is from left to right. The $\mathrm{x}$ scan rate is 1/20th the speed so that 20 vertical scans take place for each horizontal scan. In this way the probe traverses the entire focal plane. Once the end of the frame is reached, the beam is snapped back to its initial position to begin a new frame. The spectroscopic data obtained from the probe beam are filled into either a surface or a contour plot as in Figure 16b. One can consider the base of this plot to correspond to the $\mathrm{xz}$ focal plane. The data are placed into this format sequentially in the same order 
a

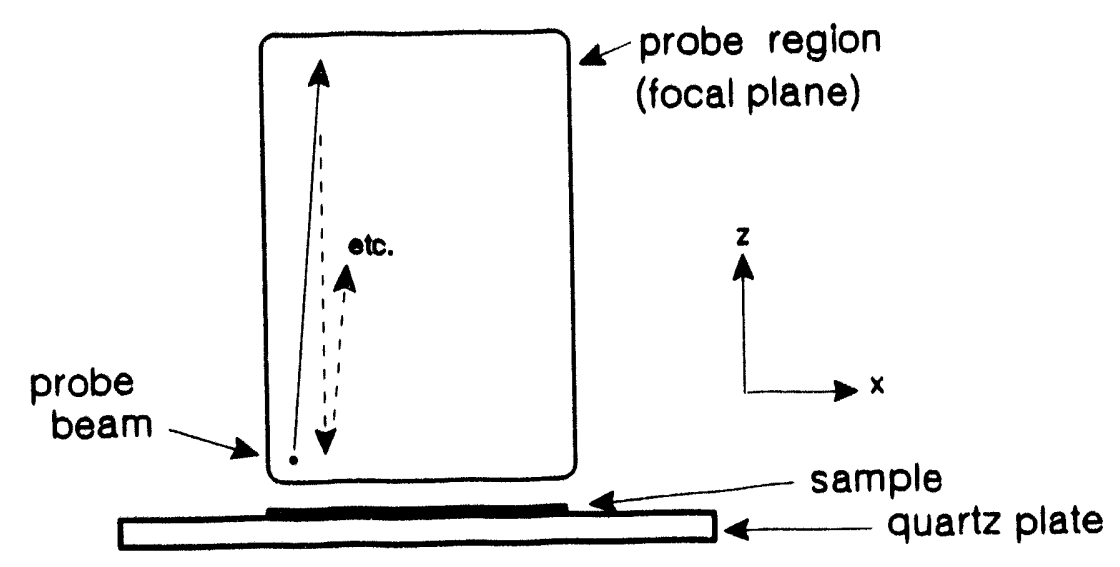

b

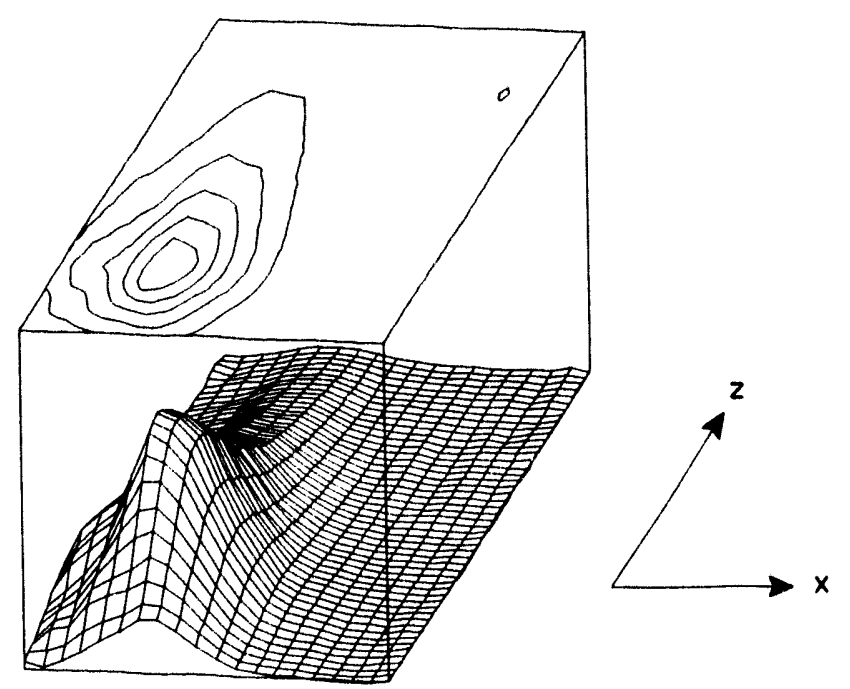

Figure 16. Depiction of imaging technique; a) orientation of scanning probe, b) graphical representation of spectroscopic signal from scanner 
as the probe beam motion. Intensity is represented as height from the plane.

With the appropriate transducer, the desired spectroscopic information can be collected. The use of precisely known waveforms to drive the scanning AODs allows one to transform temporally collected data into spatially meaningful results. This presentation process generally involves portraying the spectroscopic signal as the third dimension in a plot where the first and second dimension are spatial. Either a threedimensional surface plot or a contour plot will convey the necessary information. One can envision the data being filled into a two dimensional grid in the same manner in which the probe beam scans the image plane. One note to be kept in mind is the fact that the spectroscopic data are sequential in nature.

This scanning system allows the imaging of laser generated plumes. The distinctive feature is that these probes can follow the neutral as well as the ionic forms of the vaporized species. If fragmentation involves destruction of the chromophore, one can even measure the extent of fragmentation." 


\section{CHAPTER 7. ABSORPTION STUDY}

Previnus work in this laboratory has demonstrated the use of the AOD scanning system discussed in Chapter 6 for absorbance imaging of laser desorbed (LD) molecules." It was reasoned that this approach could be extended to matrix-assisted laser desorption as well as the more general LD. A significant effort was made to obtain absorbance images of MALD generated plumes.

\section{Experimental}

For preliminary work, the laser probe was not scanned, but left stationary. The purpose for this was to simplify the experiment. The use of the AOD scanners discussed in Chapter 6 significantly increases the degree of difficulty and complexity of the experiment. Not only is the data collection more complex, the data reduction is complicated considerably. Using knowledge from previous imaging work, we were reasonably certain of being able to estimate the plume positions. By aiming the probe laser beam at the most likely area, a signal would be generated as the plume passed through the probe.

Matrices were chosen based on previous utility in MALD-MS. Those used were caffeic and ferulic acids. Dyes used were disodium fluorescein and rhodamine 6G. They were chosen because they have large molar absorptivities at $488 \mathrm{~nm}$, the probe wavelength. Solutions were prepared in methanol with about a 200 -fold molar matrix excess. Samples were prepared by casting $10 \mu \mathrm{L}$ solution aliquots onto warmed quartz plates. This method produced rather uniform circular films, although a thicker crust 
formed at the edge. A representative sample contained a matrix concentration of $\sim 1000 \mathrm{nmol} / \mathrm{cm}^{2}$.

The $\mathrm{N}_{2}$ desorption laser delivered single pulses with energies of $\sim 2 \mathrm{~mJ}$. Backside geometry was used with each pulse completely removing the sample from the plate. The amount of dye desorbed was several hundred nanograms.

The experimental setup is diagrammed in Figure 17. In this arrangement an argon ion laser (Laser Ionics, model 554A) lasing at $488 \mathrm{~nm}$ was used as the probe. A 50:50 beamsplitter was used to direct half of the argon ion beam to PD1, a reference photodiode. The remainder of the beam interrogated a $2 \mathrm{~mm}$ cross-section located about $5 \mathrm{~mm}$ above the sample plate. Once past the plume region, the beam is directed to PD2, the signal photodiode. The reference signal is amplified (Tektronix, model 7A15/7704A) and sent to a Data Precision D6000 digital oscilloscope. Both photodiode signals are sent to a difference amplifier (Tektronix, model 7A22/7904) and subsequently digitized.

\section{Results}

The desorption of Rhodamine $6 \mathrm{G}$ in a ferulic acid matrix showed a definite attenuation of the probe beam. Transient signals approaching 0.35 absorbance units were recorded. These signals persisted over nearly $1 \mathrm{~ms}$, the lifetime of previously observed laser plumes. ${ }^{11}$ Samples of matrix without incorporated dye were analyzed as controls. These samples also attenuated the probe giving signals of nearly the same magnitude. It is reasoned that the primary attenuation mechanism is scattering of the 


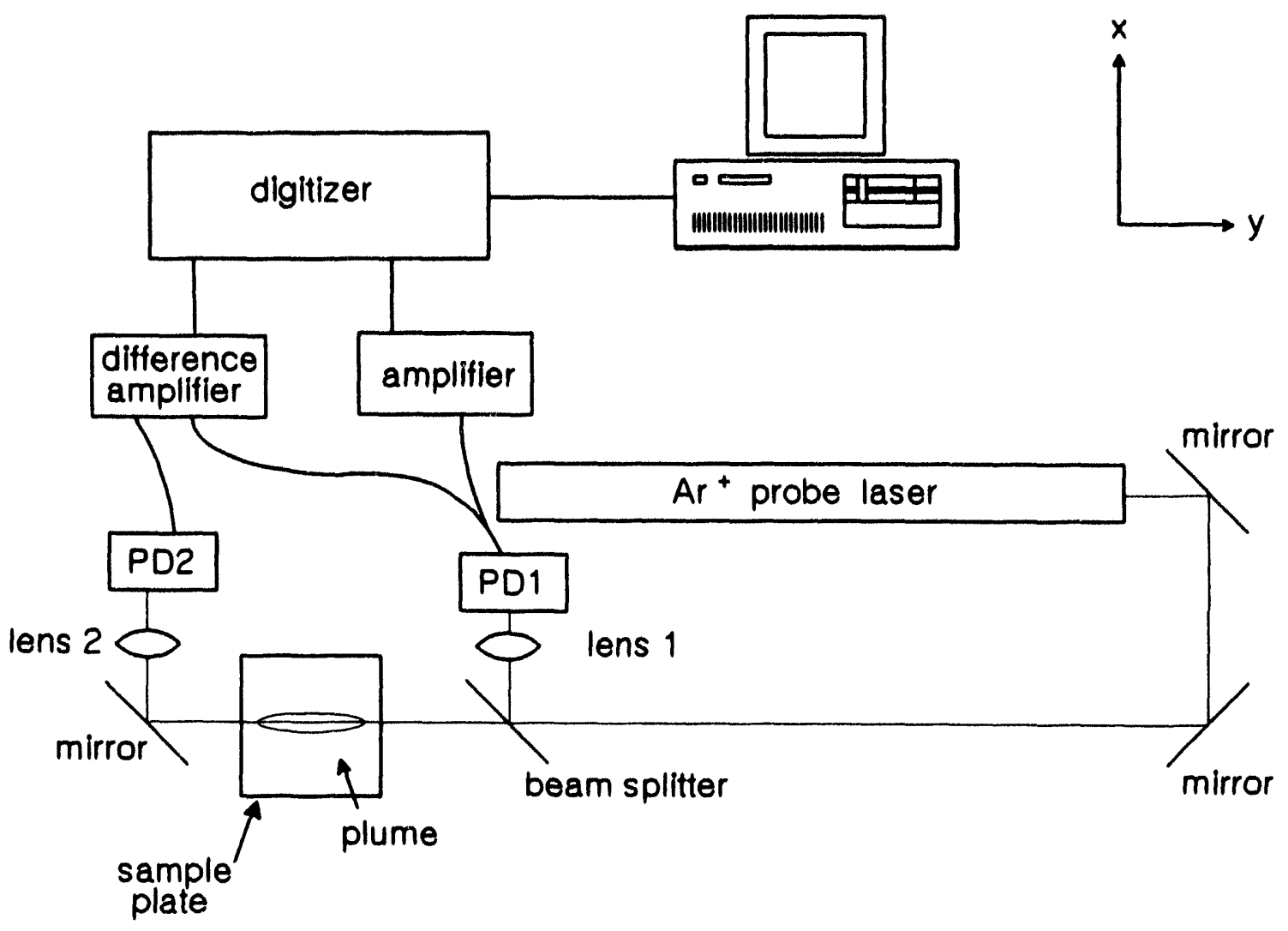

Figure 17 Experimental setup for absorbance study; $P D=$ photodiode 
probe beam by particles in the desorbed plume. Signals observed in the dye samples typically exceeded the blanks by about $25 \%$ leading to the possibility that absorbance by the dye was indeed occurring. However, the large attenuation of the probe by the matrix combined with the event to event variability of the plumes led to the conclusion that further efforts to image MALD plumes by absorbance would be fruitless. Rough calculations based on amount of desorbed chromophore (several hundred $\mathrm{ng}$ ) versus that in previous LD work $(\sim 1 \mu \mathrm{g})^{11}$ indicate that the amount of MALD chromophore is an order of magnitude less. Even though chromophore destruction ought to be reduced, this amount may still be too small to measure. The project was therefore abandoned. 


\section{CHAPTER 8. FLUORESCENCE PLUME IMAGING}

Attempts to image MALD generated laser plumes by optical absorbance met with little success as discussed in Chapter 7. This is taken to be because MALD generates substantially lower concentrations of the species of interest than LD. In this chapter the use of laser-excited fluorescence to provide spatial and temporal maps of the plumes generated by MALD is discussed. The information available is similar to that obtained by absorption probes, but with the high sensitivity needed for MALD events.

\section{Instrumental}

The probe setup is shown in Figure 18. The desorption strategy was as outlined in Chapter 2. The orientation of the desorption line was lengthwise parallel to the probe beam.

The laser-generated plume was probed with an argon-ion laser (Laser Ionics, Orlando, FL, model 554A) lasing at $488 \mathrm{~nm}$. Two mirrors were used to direct the probe beam into the first acousto-optic deflector (AOD). The first AOD (Isomet, Springfield, VA, model 1205-C) scanned the probe beam in the horizontal direction. The output from the first AOD was sent through a second AOD (IntraAction, Belwood, IL, models ADM-150 and DE-150) which scanned the probe beam in the vertical direction.

The AOD's were driven by asymmetric triangular waveforms at $200 \mathrm{kHz}$ vertically and at $10 \mathrm{kHz}$ horizontally (Wavetek Corporation, San Diego, CA, models 162 and $182 \mathrm{~A}$, respectively). The waveform asymmetries produced unidirectional scans 


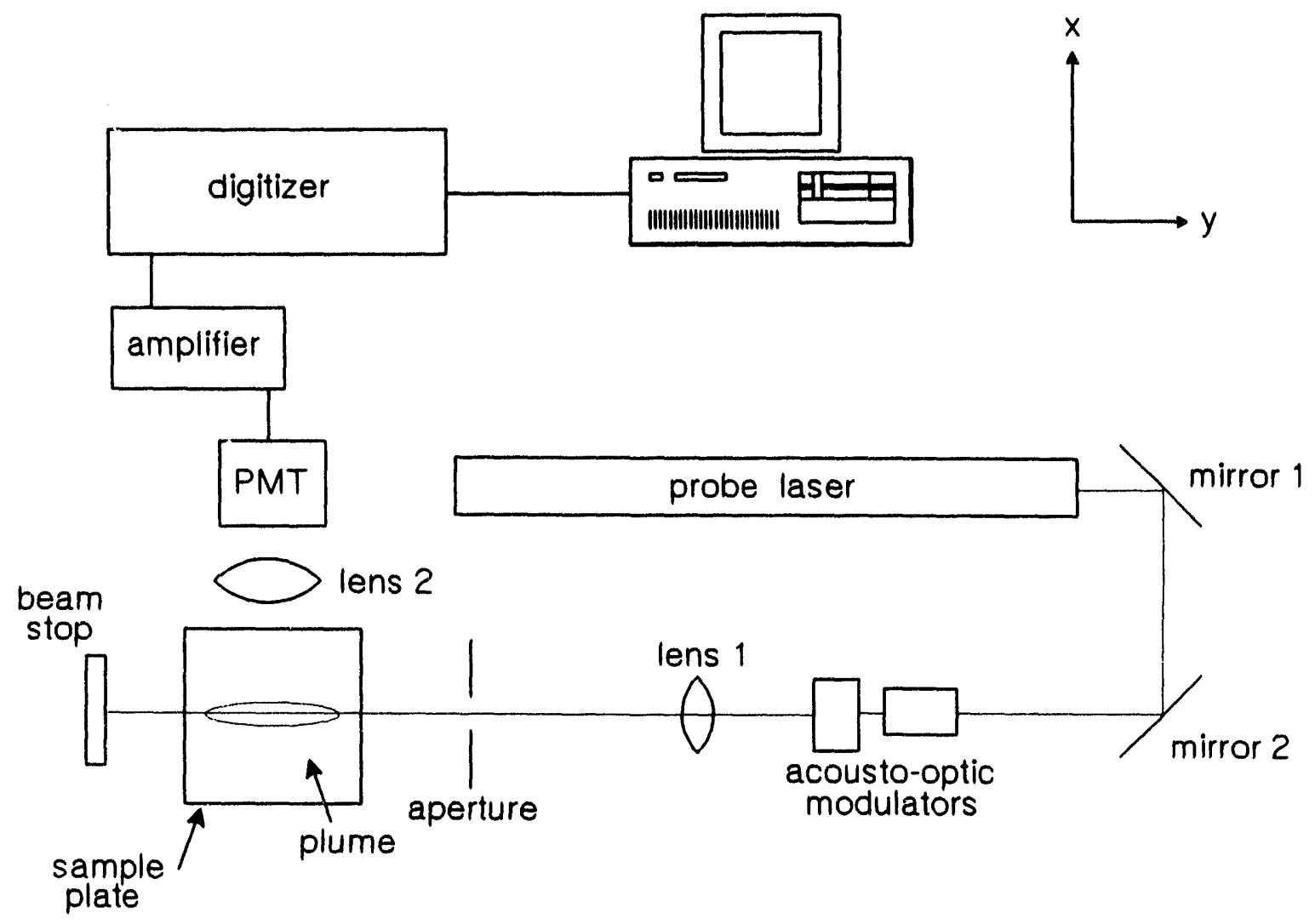

Figure 18. Block diagram of argon-ion probe for fluorescent plume mapping (see text for explanation) 
with periods of $5 \mu$ s vertically and $100 \mu$ s horizontally. During the last $5 \%$ of each sweep the beam was reset, or returned to its starting position. The vertical waveform generator was gated with TTL output pulses from the horizontal waveform generator. The horizontal waveform generator was itself gated by the SYNCH OUT signal from the $\mathrm{N}_{2}$ laser.

In order to map the largest area possible, a long focal length $(200 \mathrm{~cm})$ lens was used to focus the probe beam. It has been documented ${ }^{94}$ that AOD's can have significant cylindrical lensing effects when operated at high frequency. The lower frequency horizontal AOD has an inconsequential focal length of more than $30 \mathrm{~m}$. However, the higher frequency vertical AOD has a focal length of $\sim 1.7 \mathrm{~m}$ which is significant in comparison to the $200 \mathrm{~cm}$ lens. Because the AOD lensing characteristics are different in each dimension, the optimum focal lengths for the two scanners are not the same. Empirically, the best resolution was obtained with the spherical lens positioned $\sim 120 \mathrm{~cm}$ from the probe region. Resolution was determined by measuring the probe beam intensity transmitted through a grid at the focal point. The photodiode measurement demonstrated a resolution of about $20 \times 24$ spots in a $16 \mathrm{~mm}$ vertical $\times$ $10 \mathrm{~mm}$ horizontal scan. Naturally, optimization between the resolution and the image size can be changed by proper focusing. An aperture was placed so as to block the zeroth and second orders from the AOD's.

A $10 \mathrm{~cm}$ diameter $\times 10 \mathrm{~cm}$ focal length spherical lens was used to collect the fluorescence signal from the entire probe region onto a photomultiplier tube (Products for Research, Danvers, MA, model 56 AVP). To minimize scattered radiation the 
PMT was p'aced normal to the probe beam. In addition, a $500 \mathrm{~nm}$ cutoff filter and a $488 \mathrm{~nm}$ holographic Raman edge filter (Physical Optics Corp., Torrance, CA) were used to discriminate against scattered probe light. Finally, an interference filter was used to complete wavelength discrimination. A variety of interference filters were used including $520 \mathrm{~nm}, 550 \mathrm{~nm}$, and $577 \mathrm{~nm}$. The specific filter was selected so as to yield the maximum fluorescence signal for each sample. The PMT signal was terminated at $1 \mathrm{k} \Omega$ and sent to a digital oscilloscope (LeCroy, Chestnut Ridge, NY, model 9410). The digitizer was operated at $4 \mathrm{MHz}$ with 8-bits of vertical resolution. For each desorption event 8000 data points were recorded. This allowed twenty sequential 100 $\mu$ s two-dimensional images to be collected. Therefore, each plume was monitored for a total of $2 \mathrm{~ms}$. Data were dumped to an IBM-AT microcomputer through an IEEE-488 interface (Capital Equipment Corp., Burlington, MA, model PC-488) for subsequent evaluation. Data acquisition was triggered in the single event mode by the SYNCH OUT signal from the desorption laser and commenced $\sim 12 \mu \mathrm{s}$ after the desorption event.

The AOD's efficiency is not necessarily constant over the length of a scan, with the efficiency falling off at the ends. To allow for correction of AOD efficiencies, a portion of the post-plume probe beam was reflected to a $1 \mathrm{~cm} \times 1 \mathrm{~cm}$ photodiode (Hamamatsu, Middlesex, NJ, model S1790-01). Normalization of the PMT signal with respect to the reference photodiode signal showed no detectable change from the raw signal. We therefore concluded that the scanning efficiency was constant over the plume region of the two-dimensional maps. 


\section{Fluorescent Dyes}

Reagents and sample preparation

Dyes were chosen to represent a variety of absorptivities at the desorption wavelength $(337 \mathrm{~nm})$ as well as reasonable fluorescence quantum efficiency at the probe wavelength $(488 \mathrm{~nm})$. The dyes investigated included coumarin 6 and coumarin 334 (Eastman Kodak, Rochester, NY), disodium fluorescein (Lambda Physik, Acton, MA), and rhodamine 6G (Exciton, Dayton, $\mathrm{OH}$ ). Imaging was done with caffeic, ferulic, and gentisic acids. Matrices were obtained from Aldrich Chemical. All reagents were used as received.

Solutions were prepared by dissolving matrix in stock methanolic dye solutions. All samples were prepared on $2 \times 2 \times 1 / 16^{n}$ quartz plates. Most samples were prepared by spin-coating $10 \mu \mathrm{L}$ aliquots. For the thickest films, solutions were cast directly onto quartz and dried under a gentle stream of room temperature air.

Results

Ferulic acid was found to be the most effective matrix and will be discussed in detail here. Caffeic and gentisic acids gave similar results, but the signals were factors of two and three smaller, respectively. In preliminary work, sinapinic acid was found to desorb quite well. It was not studied further, however, as its solubility in methanol was too low to give an acceptable matrix/dye ratio.

Not all of the dyes investigated gave a measurable signal. Both coumarin dyes produced decent signals with coumarin 6 giving the largest. Coumarin 334 gave good 
signal to noise, although less than coumarin 6 . Rhodamine $6 \mathrm{G}$ was detectable, but only slightly above background. Surprisingly disodium fluorescein was never detected. Desorption geometry was backside in all cases. Repeated attempts were made at frontside geometry, but too little material was removed. Although a signal was sometimes observed, it was only slightly above background. No useful images were obtained.

In Figure 19 surface plots of coumarin 6 and coumarin 334 plumes are presented. The purpose here is to illustrate graphically the type of signal to noise that was possible for coumarin 6 and 334. For the purpose of visualizing the plume and its motion, contour plots were found to be more useful. The remainder of the results are presented in that format.

Figure 20 depicts a complete 20 frame run for coumarin 6 desorbed from a thin film of ferulic acid matrix. In the first frame one can see the signal from sample fluorescence excited by the desorption laser. It is not correlated with the probe position and can be considered an unwanted signal which gradually decays. If plume fluorescence excited by the probe laser is present, it is not observable above the large background. By the second frame, the initial fluorescence has largely decayed and formation of the plume is seen to begin. The third frame shows motion of the plume away from the sample surface along with increasing intensity. By this time $(\sim 250 \mu \mathrm{s})$ the center of the plume has slowed. The plume does continue to grow in intensity throughout all twenty frames. One also observes diffusion of the plum 2 throughout the remaining frames. The overall appearance of the plume does not change significantly 

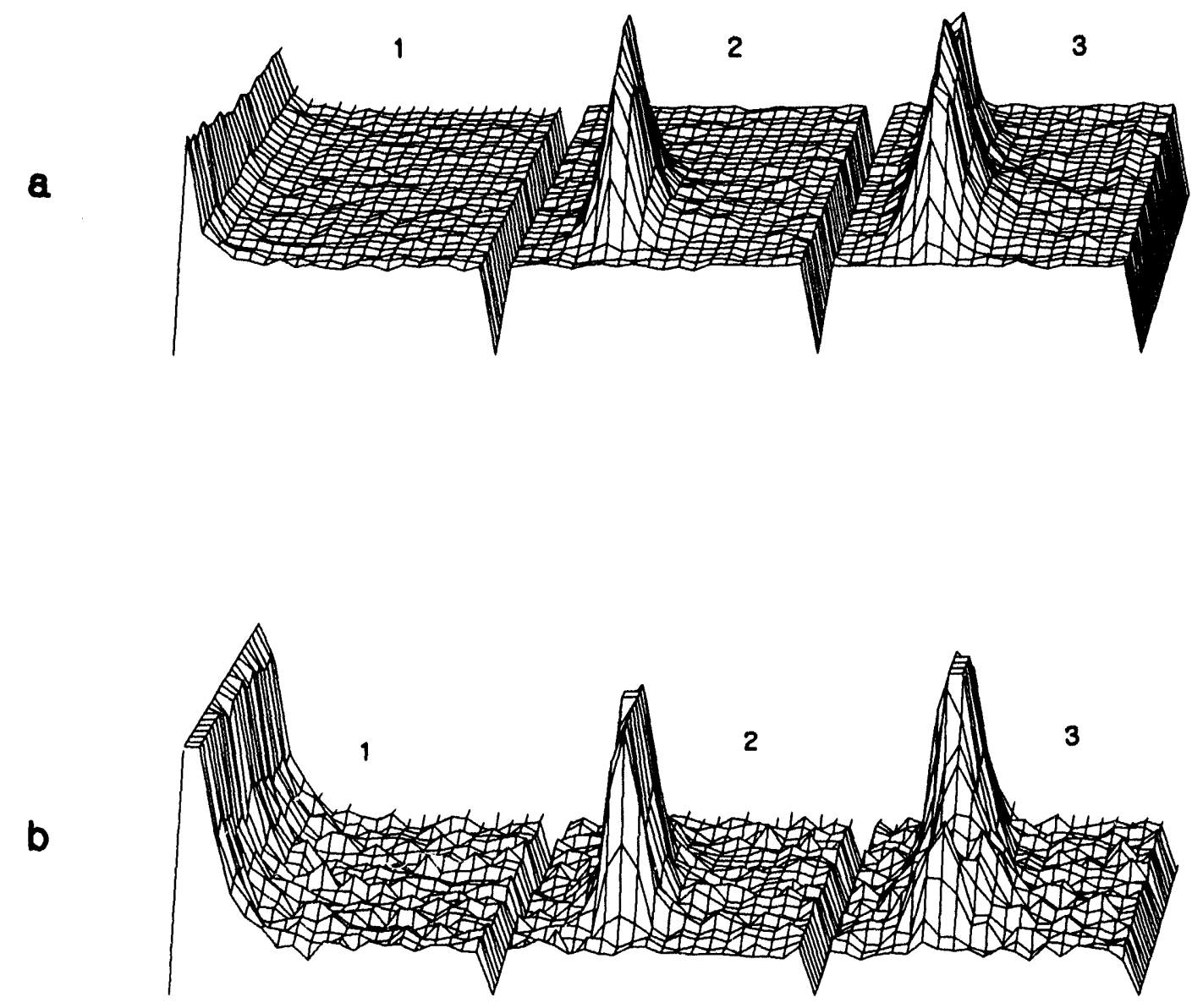

Figure 19. Sequential surface plots of coumarin 6 (a) and coumarin 334 (b) fluorescence; imaged area, $9 \mathrm{~mm}$ (vertical) $\times 8 \mathrm{~mm}$ (horizontal), $1 \mathrm{~mm}$ above the surface 

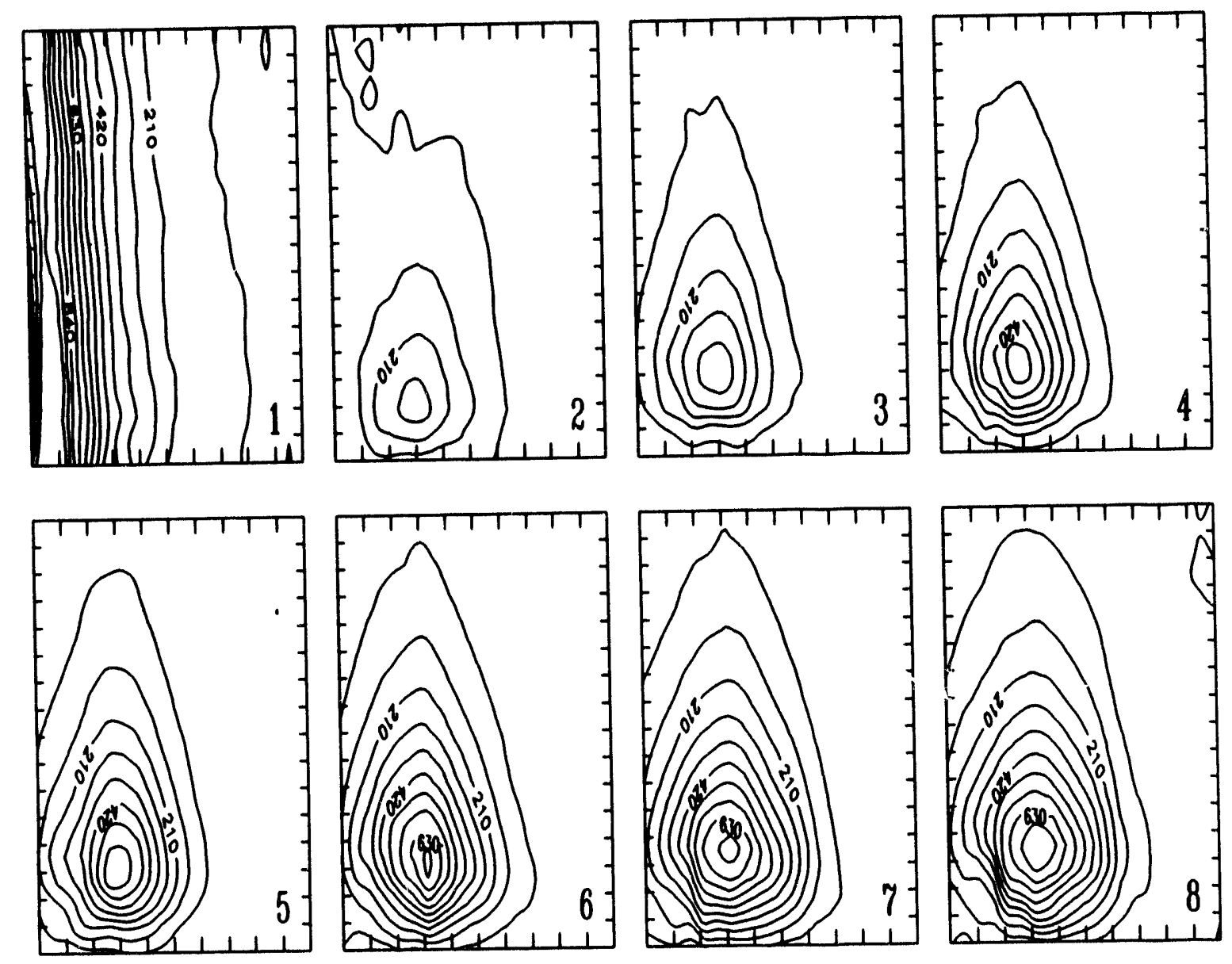

Figure 20. Complete 20 frame sequence of MALD generated plume images; $5 \mathrm{fmol}$ coumarin 6 desorbed, $30 \mathrm{nmol} / \mathrm{cm}^{2}$ ferulic acid matrix, 2000-fold molar excess; probe dimensions $10 \times 16 \mathrm{~mm}, 1 \mathrm{~mm}$ above sample; contour levels in $\mathrm{mV}$ 

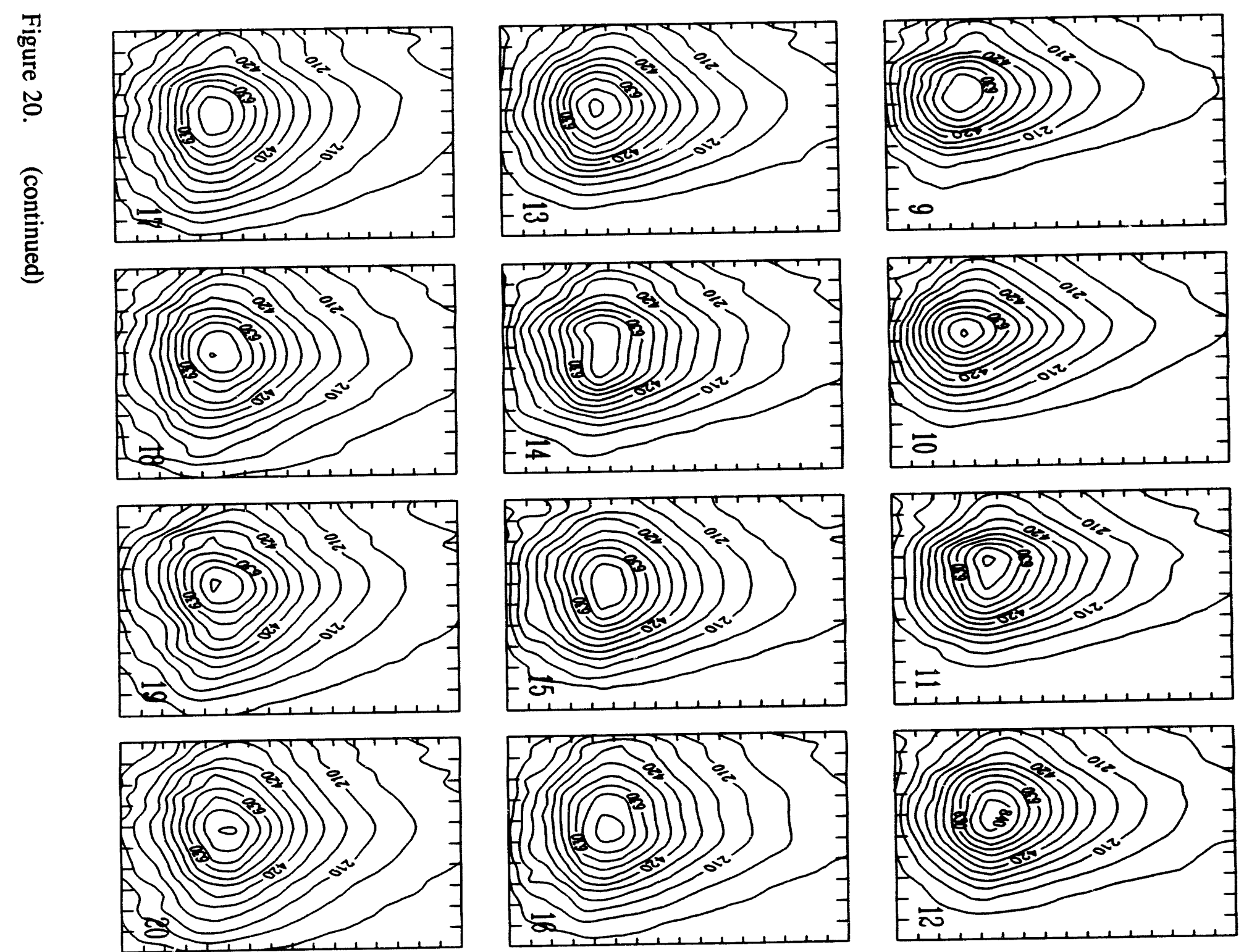
beyond frame 12. All 20 frames are presented to underscore this point. This relatively unchanging plume appearance at later times was characteristic of all plumes observed. For that reason, and to keep figures to single pages, only selected frames of the remaining plumes are presented.

The plume in Figure 20 was obtained from a relatively thin film of sample. Plume dynamics varied considerably with film thickness. In Figure 21 and Figure 22 plumes generated from thicker films are presented. One immediately notes that, unlike the thin film, the plume is visible in the first frame. This is due at least in part to the significantly greater amount of material desorbed. Because of the increase in fluorescence signal, the PMT gain could be reduced. The result is an effective increase in discrimination against desorption induced fluorescence. The second obvious difference from the thin film is the much more rapid movement of the plume away from the sample surface. By frame 3 the plume center is $9 \mathrm{~mm}$ above the surface. This is over twice as far as the thin film plume travelled in the same time period. Yet another difference is the degree of lateral dispersion. While the thinnest film appeared to disperse laterally in roughly the same degree as along the surface normal, the thicker film seems to be somewhat more focussed along the normal. A similarity is that by frame 3 the rapid plume motion has ceased. As in Figure 20 the plume continues to move, but at a reduced rate. The thickest coumarin $6 /$ ferulic acid film is shown in Figure 22. Here the initial rapid motion is even nore pronounced. It does not subside until frame 6 where the plume center is located $14 \mathrm{~mm}$ above the surface. Again, the plume shows less lateral diffusion than the thin $\mathrm{C} 6$ film. 

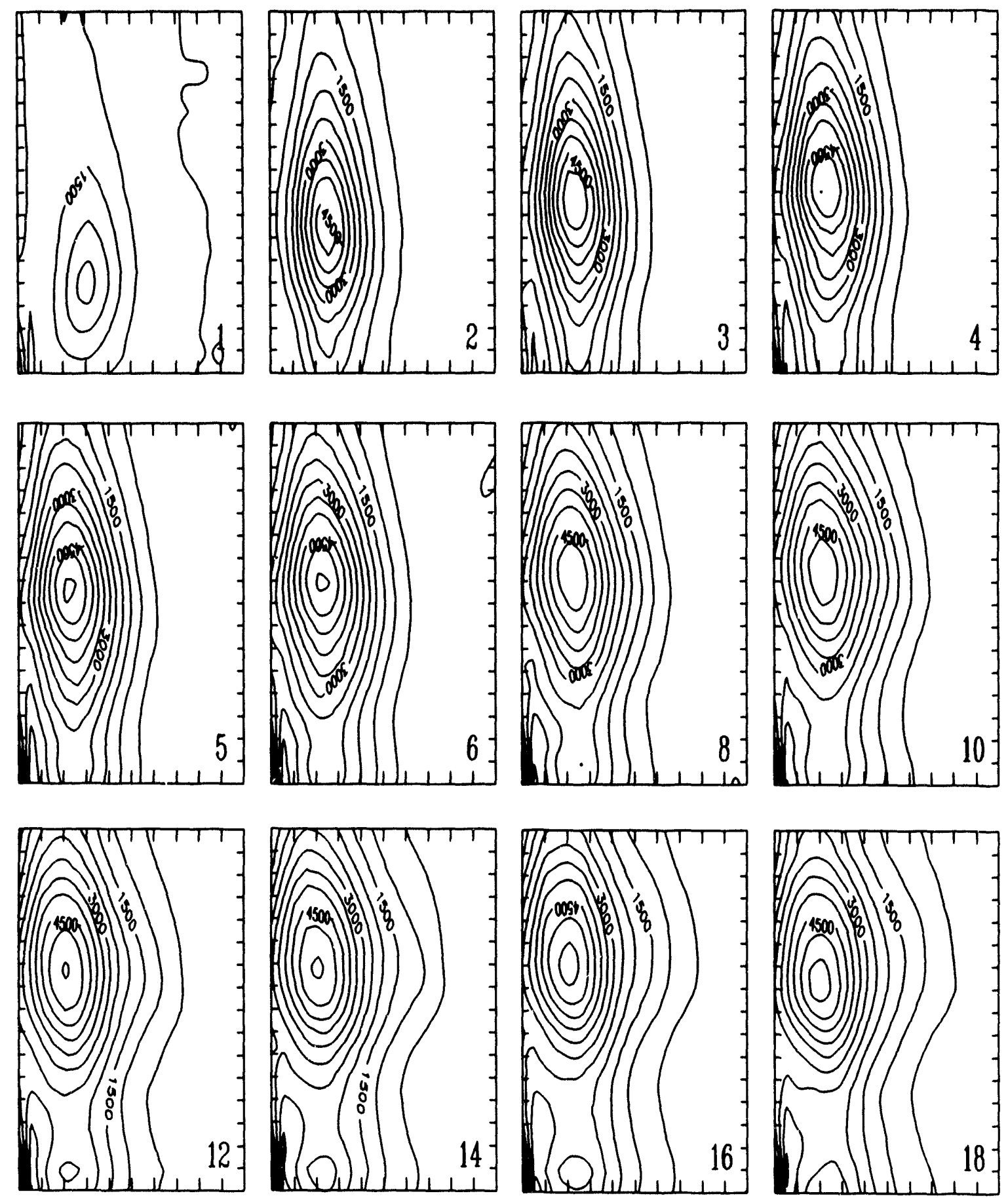

Figure 21. Selected sequential images of a MALD plume; $50 \mathrm{fmol}$ coumarin 6 desorbed, $300 \mathrm{nmol} / \mathrm{cm}^{2}$ ferulic acid matrix, 2000-fold molar excess 

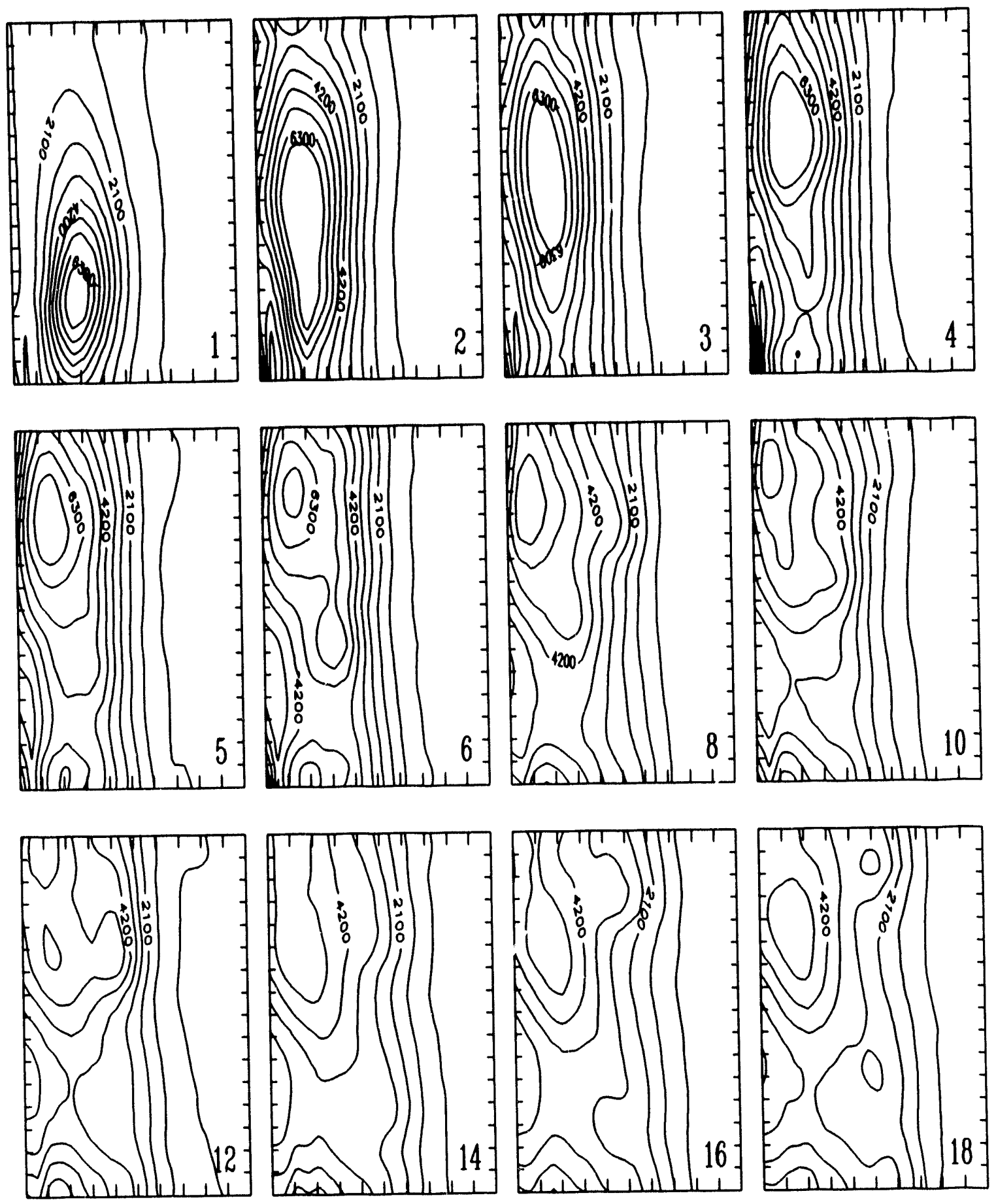

Figure 22. Sequential images of a MALD plume; $\sim 500 \mathrm{fmol}$ coumarin 6 desorbed, $\sim 1000 \mathrm{nmol} / \mathrm{cm}^{2}$ ferulic acid matrix, 2000-fold molar excess 
As mentioned before, backside desorption typically resulted in virtually complete sample removal. It was observed however that a second laser shot on the same spot would often generate a fluorescence signal. Figure 23 shows the plume formed from the second shot on the same spot as Figure 21. It is interesting that the plume motion appears the same as the thin film. The lateral diffusion is not as great, however. It is possible that the edges left from the first shot serve to focus the second plume. It is possible this plume arises from sample residue which recondensed from the first desorption event. Other possible explanations are that the slide was not totally cleaned with the first shot or that the plume is generated from the edges of the remaining sample. The amount removed was estimated based on relative fluorescence intensity. By calculating the integrated fluorescence for each frame one can get a sense of the relative fluorophore populations. Figure 24 shows normalized fluorescence by frame for the three coumarin $6 /$ ferulic acid films. For the thick film, fluorescence intensity grows to a maximum at $\sim 600 \mu \mathrm{s}$. This agrees well with the time of maximum absorbance signal in previous work ${ }^{11,95}$, showing that the velocities of the vaporized materials are comparable. As film thickness decreases, however, the maximum fluorescence signal takes longer to develop. We note that all of these results are representative of plume velocities in atmospheric-pressure gas, and are therefore substantially slower than velocities observed in vacuum. ${ }^{61}$ The earlier time of maximum fluorescence and the greater distance traveled at early times suggest that the fluorophores are desorbed from the thicker films with greater velocities.

In Figure 25 and Figure 26 we show plots of total fluorescence as a function of 

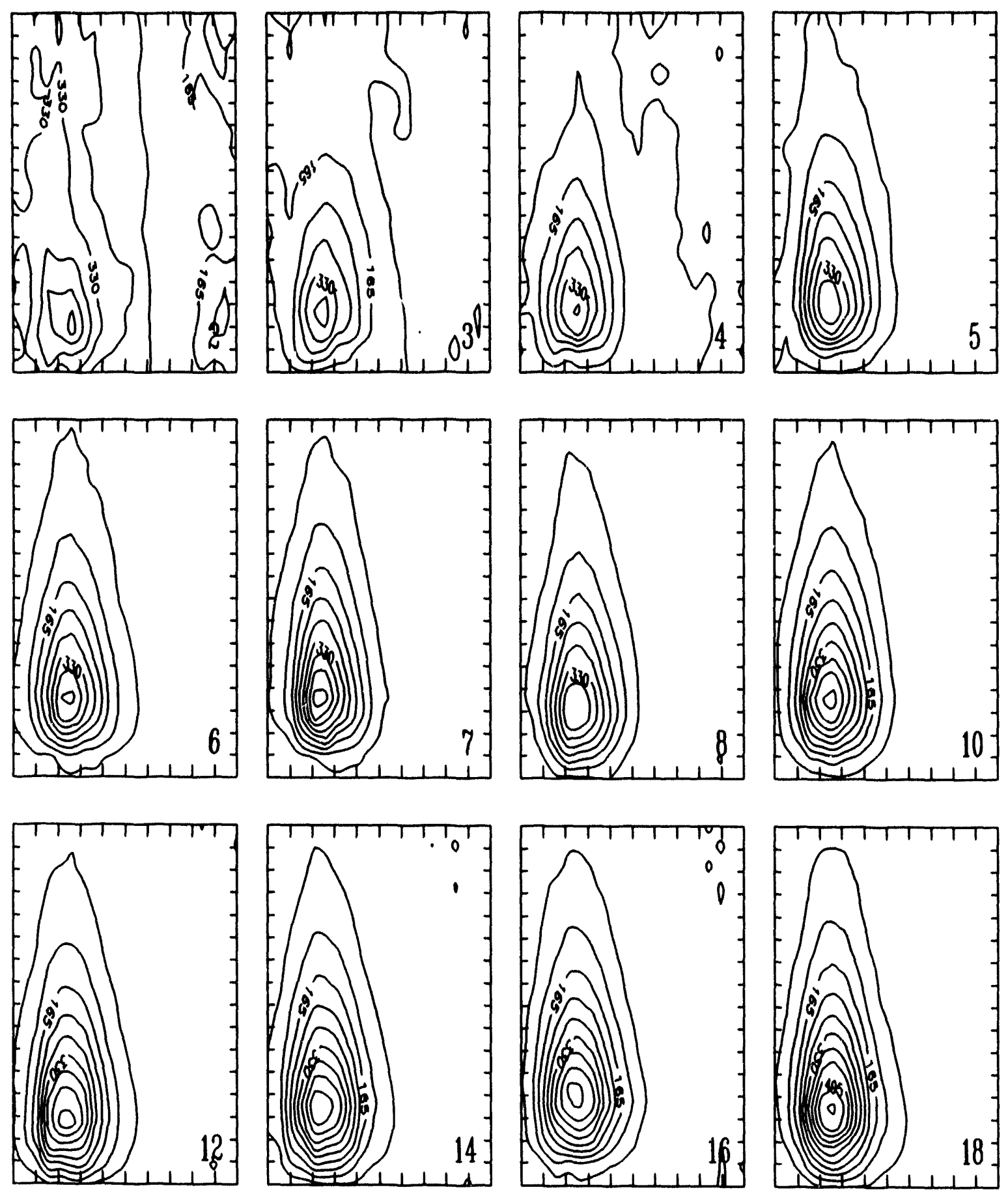

Figure 23. Selected sequential images of a second shot MALD plume; $\sim 50 \mathrm{fmol}$ coumarin 6 desorbed, ferulic acid matrix, 2000-fold molar excess 


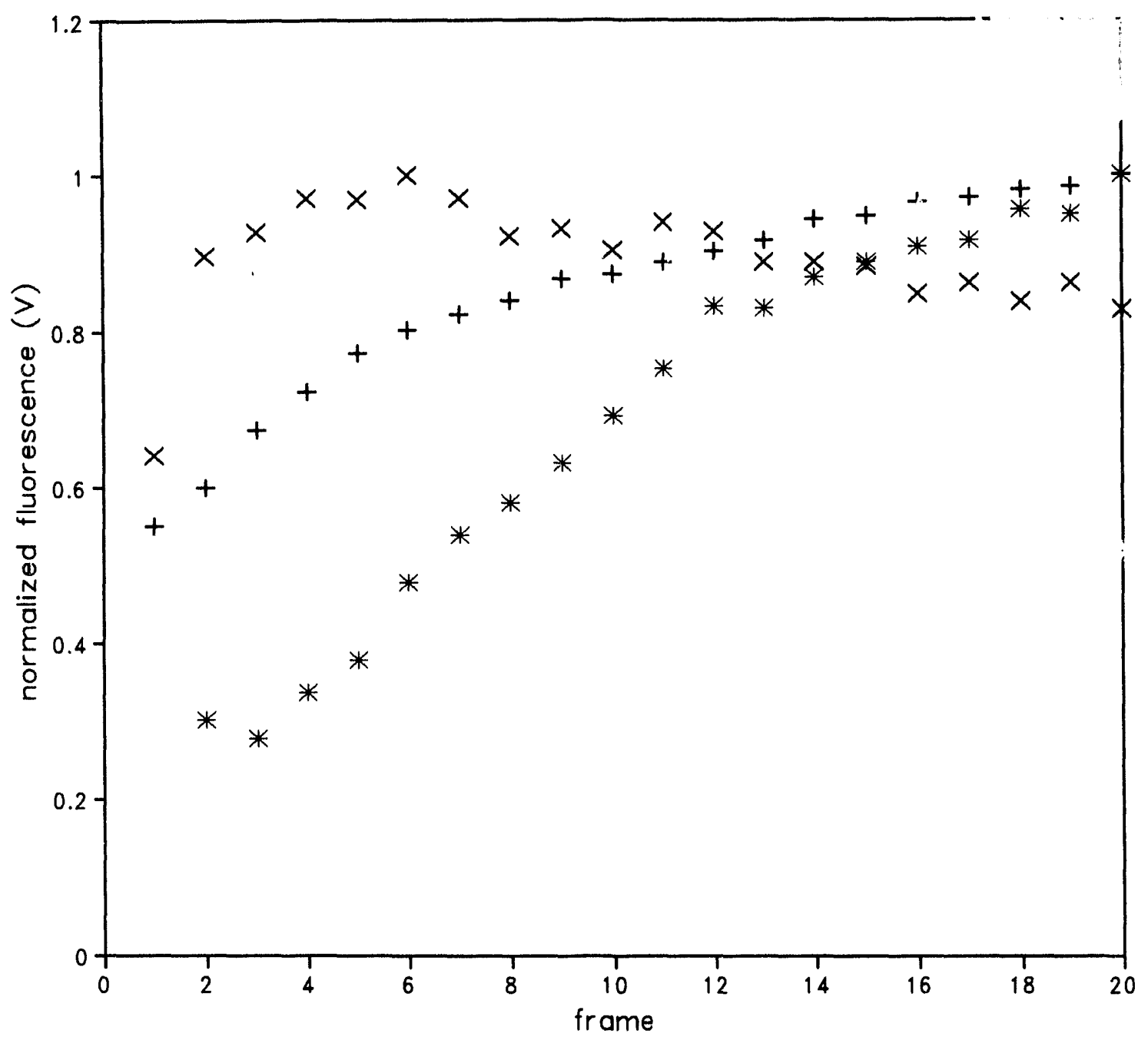

Figure 24. Normalized fluorescence by frame for backside desorption of coumarin 6; ferulic acid matrix, $30 \mathrm{nmol} / \mathrm{cm}^{2}(*), 300 \mathrm{nmol} / \mathrm{cm}^{2}(+), \sim 1000$ $\mathrm{nmol} / \mathrm{cm}^{2}(x)$ 


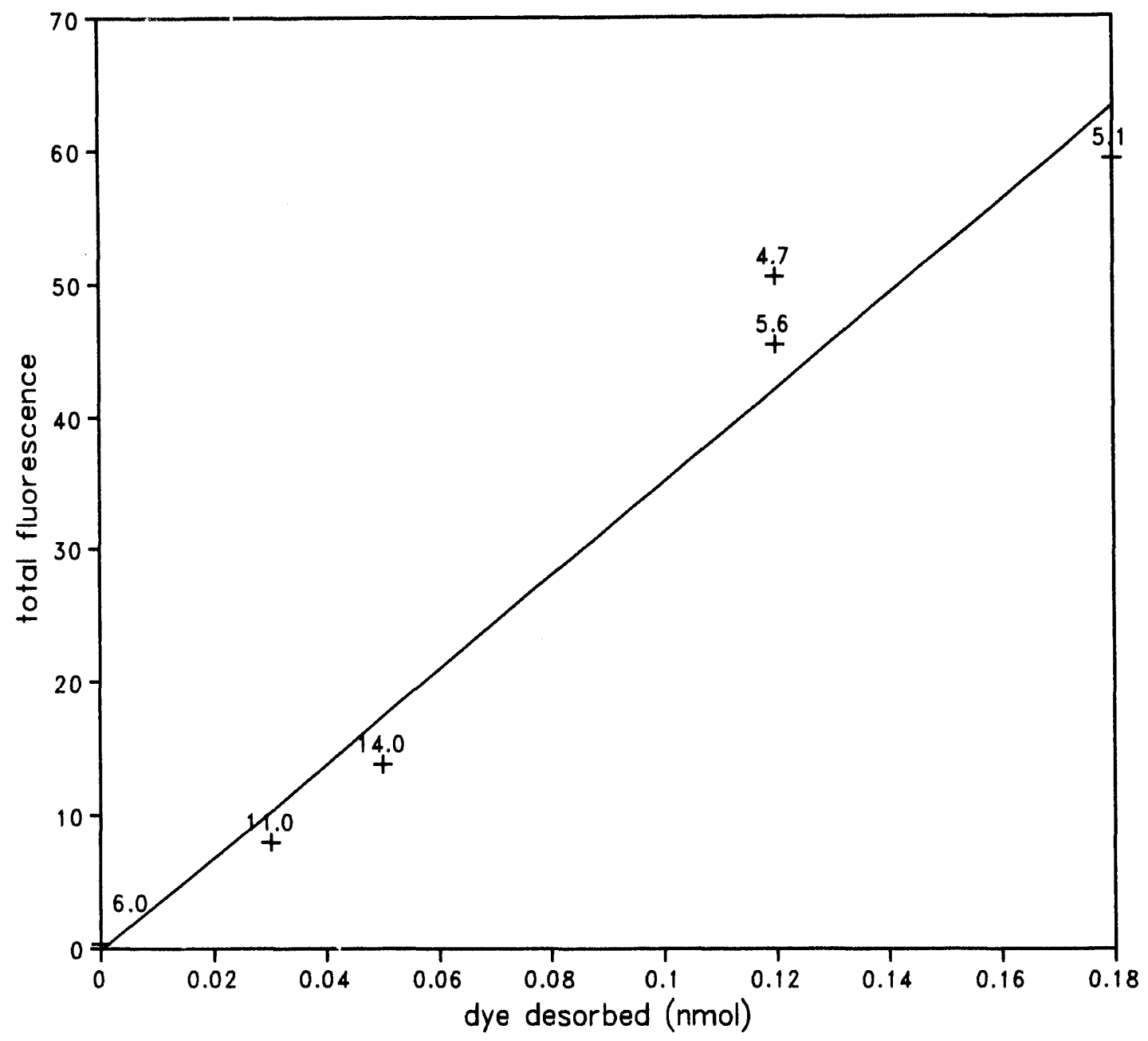

Figure 25. Backside desorption of coumarin $6 ; 70 \mathrm{nmol} / \mathrm{cm}^{2}$ ferulic acid matrix, 2000-fold molar excess, labels represent desorption irradiance in $\mathrm{MW} / \mathrm{cm}^{2}$ 


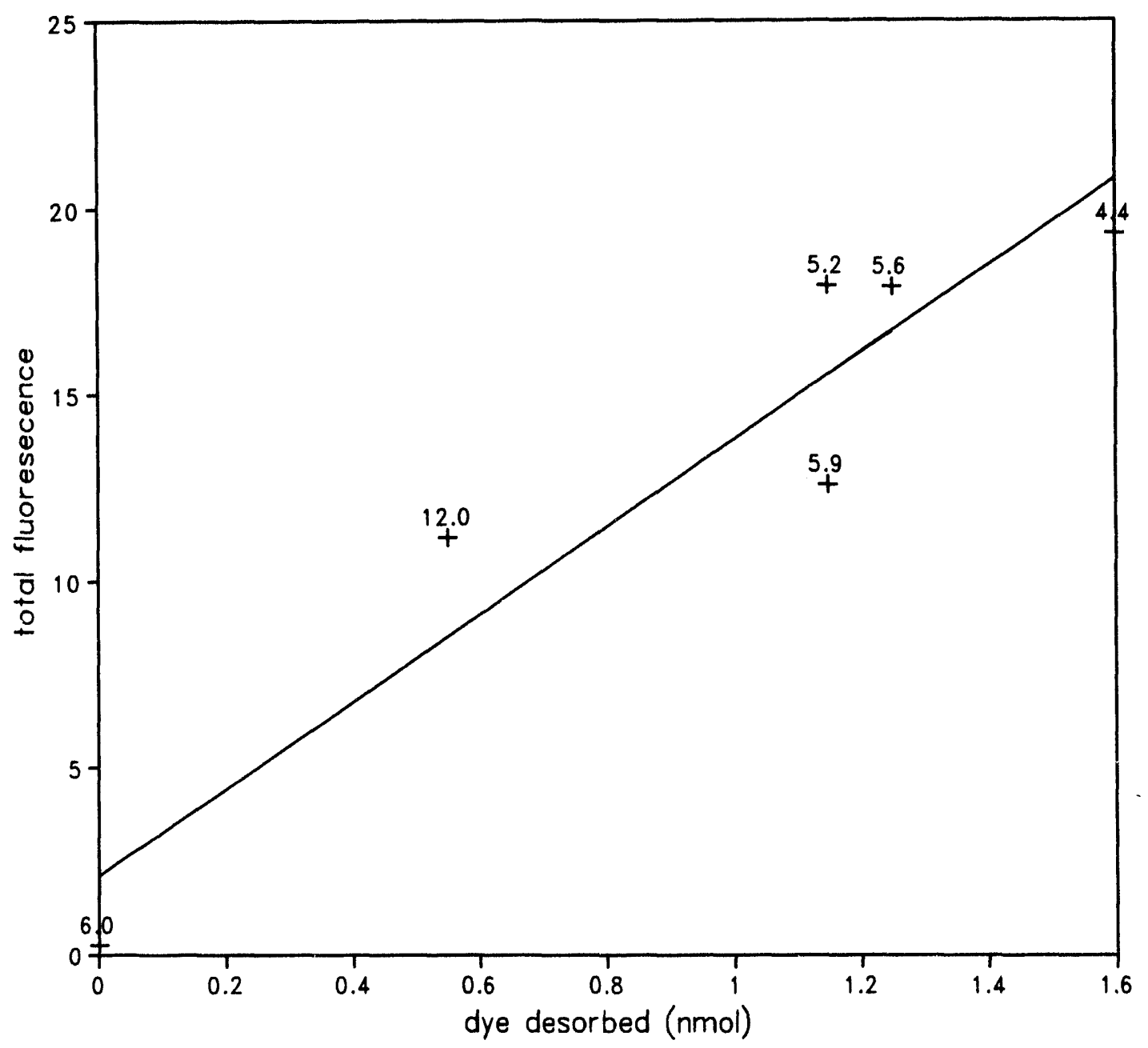

Figure 26. Backside desorption of coumarin $334 ; 70 \mathrm{nmol} / \mathrm{cm}^{2}$ ferulic acid matrix, 100 -fold molar excess, labels represent desorption irradiance in $\mathrm{MW} / \mathrm{cm}^{2}$ 
the amount of dye desorbed. The amount was determined from the sample area cleaned off by the laser for that particular desorption event and the estimated amount of dye per unit area of the film deposited. These plots are linear $(r=0.94$ and 0.98 for $\mathrm{C} 3$ and C6, respectively) but not correlated with laser irradiances which range from 4.4 to 14 $\mathrm{MW} / \mathrm{cm}^{2}$. If, in fact, the desorption laser were causing destruction of fluorophore, changes in irradiance would cause non-linearity, as was observed in reference 11 . The indication here of no sample fragmentation (at least none involving the conjugated part of the fluorophore) agrees with typical MALD-MS work which does not detect analyte fragments under these conditions.

\section{Dye Labeled Proteins}

The excitement surrounding MALD is primarily due to its ability to generate intact molecular ions from large proteins and oligonucleotides. For this reason attempts were made to study fluorescence-labeled biomolecules with the imaging apparatus. As long as the level of labeling is low, the mechanisms involved should be essentially unchanged. Fluorescence imaging could offer insight into these mechanisms and suggest ways of effectively implementing secondary ionization.

\section{Reagents}

For this investigation bovine serum albumin (BSA) was chosen as a representative protein. First, it is of intermediate molecular weight $(\sim 66 \mathrm{kDa})$ and is easily within the capability of MALD. Second, it is readily available and can be purchased with fluorescent labels already attached. The BSA samples chosen were 
labeled with sulforhodamine 101 (TR-BSA), rhodamine B (RB-BSA), fluorescein isothiocyanate (FITC-BSA), and tetramethylrhodamine isothiocyanate (TRITC-BSA). Unlabeled BSA was used as a blank. All were obtained from Sigma and used as received. Protein solutions were prepared at $1 \mathrm{~g} / \mathrm{L}$ in $0.1 \%$ trifluoroacetic acid (TFA). The matrix solution was $0.2 \mathrm{M}$ ferulic acid in 50:50 methanol/water. Working solutions were prepared by mixing $200 \mu \mathrm{L}$ of protein solution with $2 \mathrm{~mL}$ of matrix solution. $100 \mu \mathrm{L}$ aliquots of this mixture were directly cast onto $2 \times 2 \times 1 / 16^{\prime \prime}$ quartz plates and dried under a gentle stream of room temperature air. Sample morphology was quite heterogeneous with lots of flakes protruding from the surface. Actual amounts of sample deposited as well as calculated mass and molar ratios are presented in Table VI.

Table VI. Sample deposit amounts and matrix excesses

\begin{tabular}{lllcc}
\hline sample & $\begin{array}{l}\text { mass } \\
(\mu \mathrm{g})\end{array}$ & $\begin{array}{l}\text { matrix } \\
(\mathrm{mg})\end{array}$ & $\begin{array}{l}\text { mass } \\
\text { ratio }\end{array}$ & $\begin{array}{l}\text { molar } \\
\text { ratio }\end{array}$ \\
\hline coumarin 6 & 0.35 & 0.39 & 1100 & 2000 \\
FITC-BSA & 14 & 3.49 & 250 & 86000 \\
TR-BSA & 40 & 1.94 & 50 & 17000 \\
TRITC-BSA & 74 & 1.94 & 26 & 9000 \\
BSA & 80 & 1.94 & 24 & 8300 \\
DNA:TOTO-1 & 10 & 0.35 & 35 & 3600 \\
\hline
\end{tabular}


Results

Dye labeling allowed the collection of decent images of MALD generated protein plumes. Fluorescence intensity from shot to shot was extremely variable. The largest signals tended to come from the thickest portions of the sample, particularly from larger crystallites which were intimately connected to the quartz surface. MALD desorption of unlabeled BSA produced no signal above background. Results are presented on the following pages. Figure 27 shows images obtained from an FITCBSA sample. These images were collected with a $520 \mathrm{~nm}$ interference filter. Figure 28 and Figure 29 show the same thing for TR-BSA and TRITC-BSA respectively. To accommodate the different emission profiles, a $550 \mathrm{~nm}$ filter was used for TR-BSA and a $577 \mathrm{~nm}$ filter for TRITC-BSA. There does not appear to be any significant difference between the different labeled proteins. This is not too surprising and bears out the supposition that dye labeling has little influence on the protein desorption.

The plume dynamics share some characteristics of each of the dye film thicknesses. Although the protein films correspond most closely to the thickest dye film in actual thickness, the vertical motion of the plume center resembles that of the thin film. The integrated fluorescence for FITC-BSA also seems to be similar to that of the thick dye film. Looking at Figure 30 it can be seen that the maximum FITC-BSA fluorescence signal appears at very late times as it does with the thin coumarin 6 film. If one compares the lateral diffusion, however, the proteins resemble the thick dye films. It should be mentioned that these images were obtained in atmosphere so any 

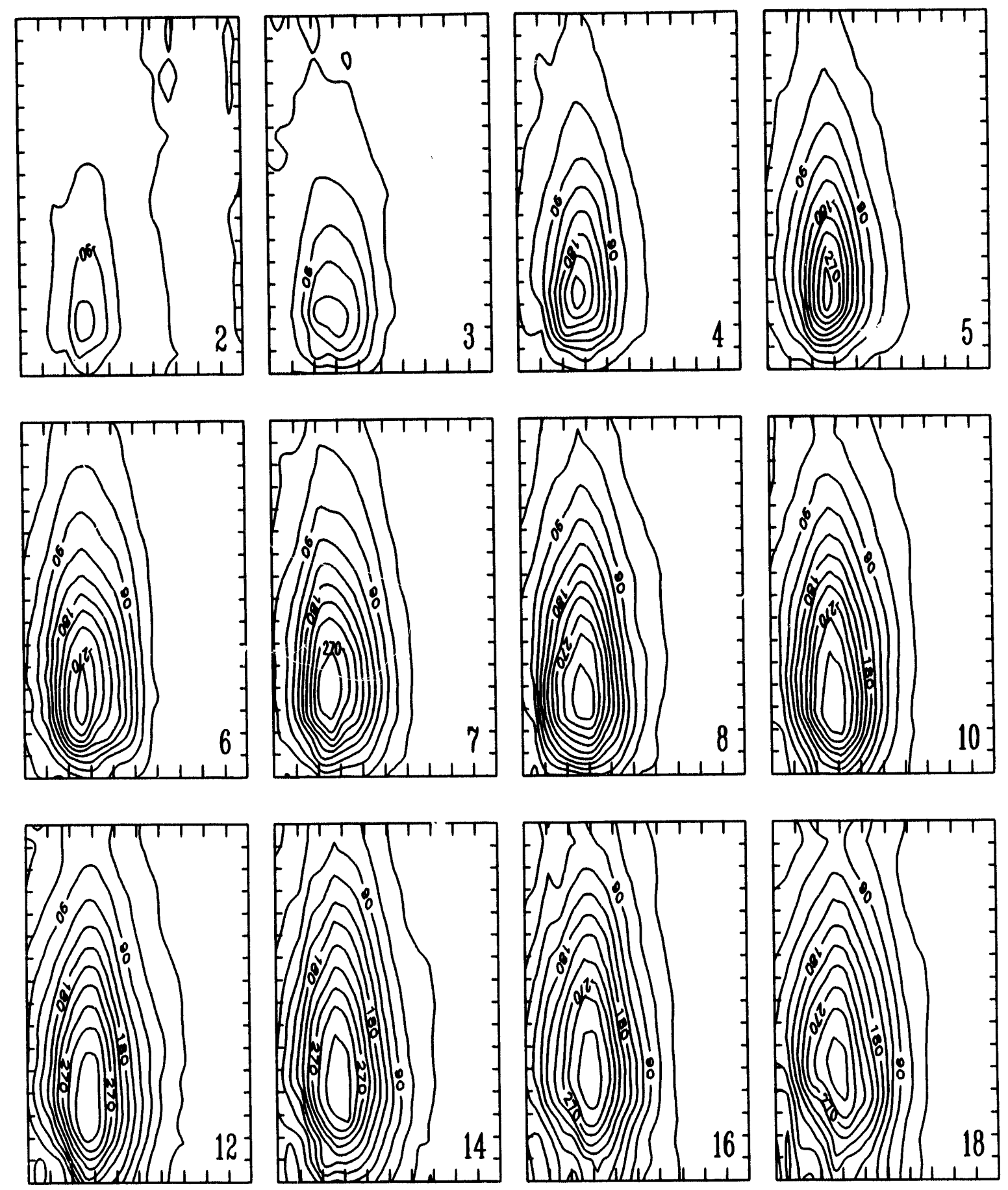

Figure 27. Selected sequential images of a MALD plume from FITC-BSA in a ferulic acid matrix 

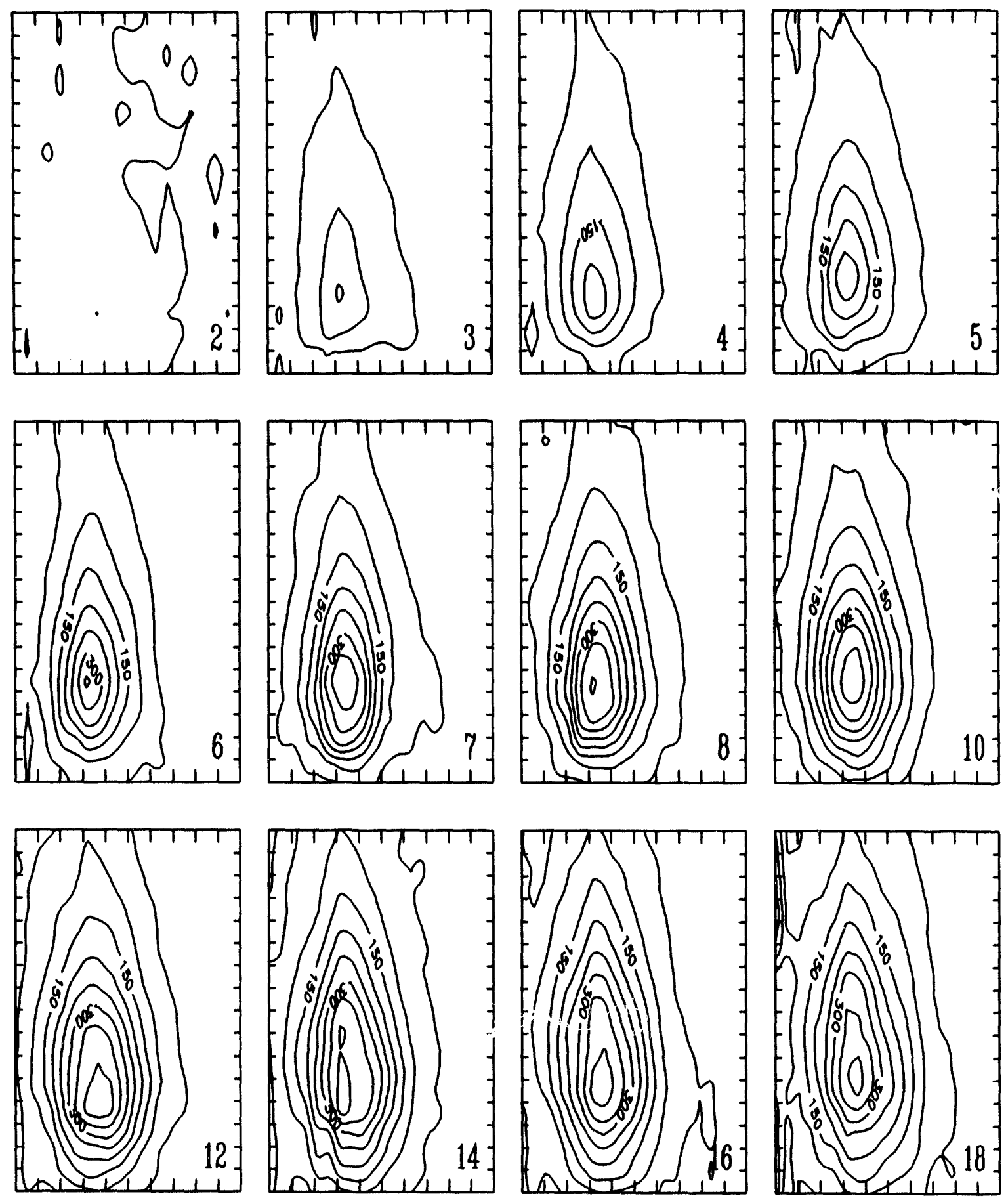

Figure 28. Selected sequential images of a MALD plume from TR-BSA in a ferulic acid matrix 

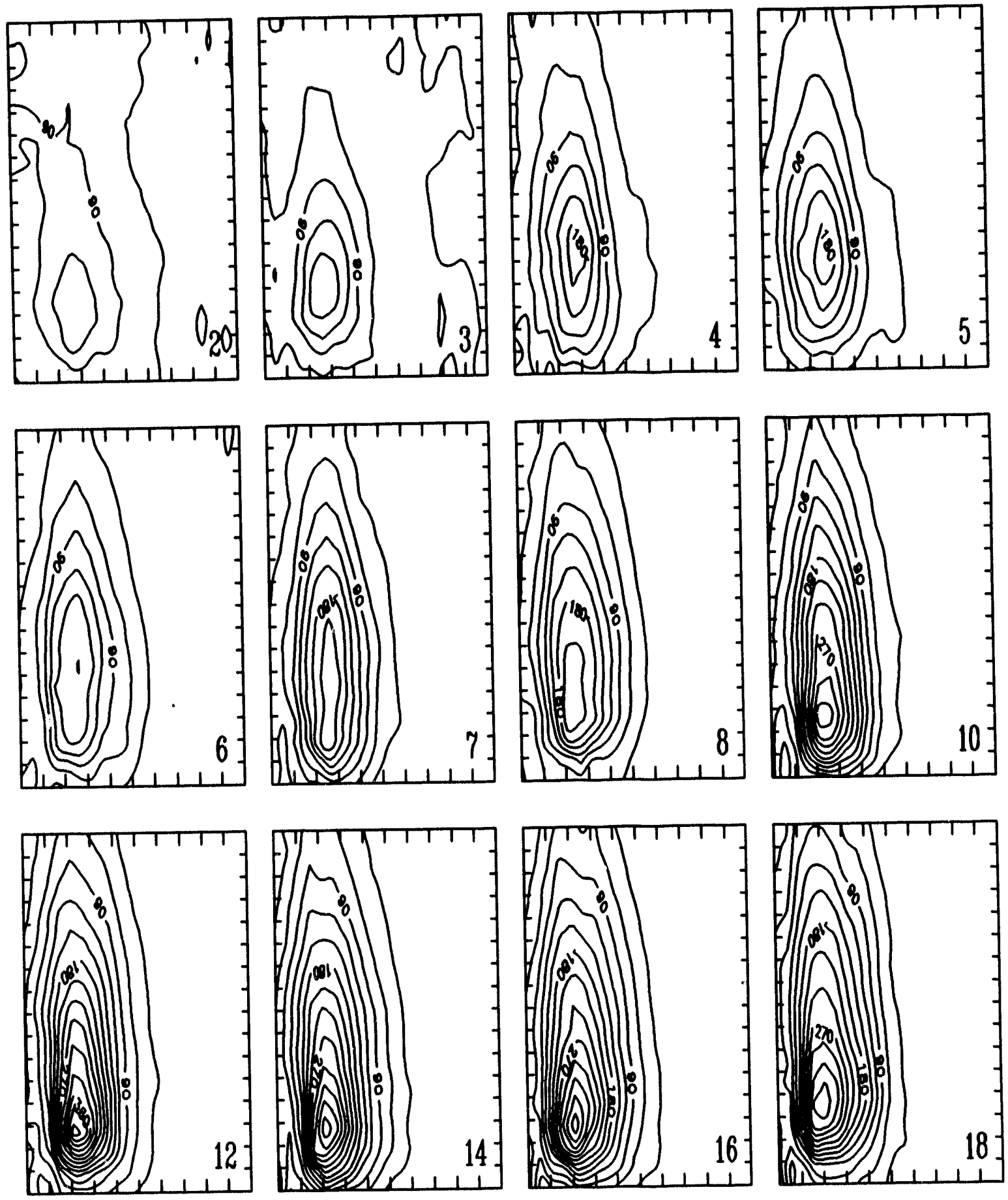

Figure 29. Selected sequential images of a MALD plume from TRITC-BSA in a ferulic acid matrix 


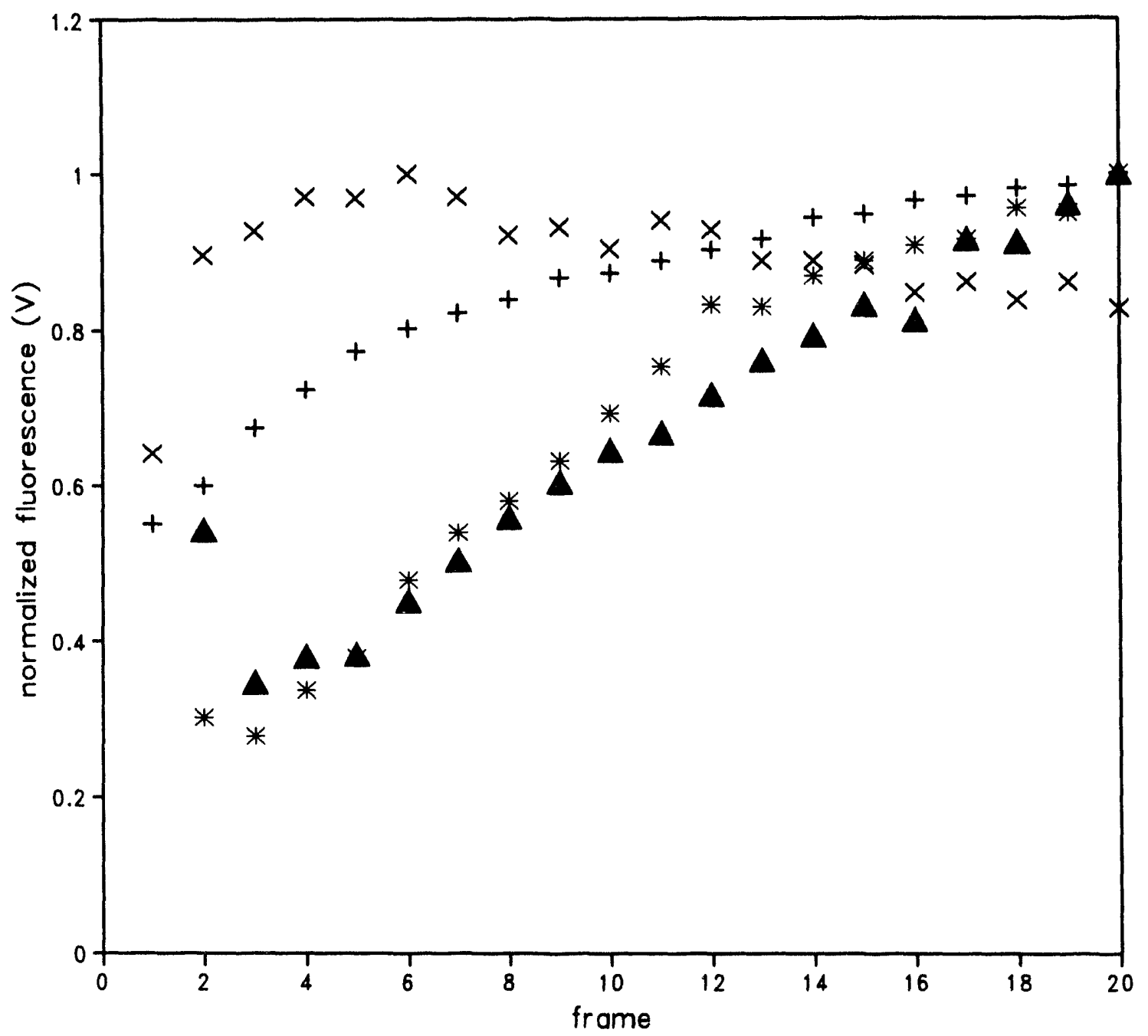

Figure 30. Normalized fluorescence by frame for backside desorption of coumarin 6 and FITC-BSA; $30 \mathrm{nmol} / \mathrm{cm}^{2} \mathrm{C} 6(*), 300 \mathrm{nmol} / \mathrm{cm}^{2} \mathrm{C} 6(+), \sim 1000$ $\mathrm{nmol} / \mathrm{cm}^{2}$ C6 $(\times)$, FITC-BSA ( $\left.\triangle\right)$ 
attempt at interpreting the data for mechanisms is not straightforward. It does seem possible to suggest that the MALD process is a function of film morphology, particularly thickness. Use of this fluorescence probe to image MALD generated plumes inside a vacuum chamber could provide significant insight into the MALD process.

\section{Dye Labeled DNA}

\section{Reagents}

DNA used was degraded free acid from Herring sperm obtained from Sigma. The molecular weight was in the 10 to $30 \mathrm{kDa}$ range. The dye reagent was TOTO-1 from Molecular Probes. TOTO-1 is an intercalating dye which exhibits a thousand-fold enhancement of fluorescence efficiency upon binding with DNA. Reagents were used as received. DNA was dissolved in tris-borate buffer, $\mathrm{pH} 8.4$, at $1 \mathrm{mg} / \mathrm{mL}$. To $1 \mathrm{~mL}$ of this solution, $31 \mu \mathrm{L}$ of stock dye solution was added. After incubating for 30 minutes, the mixture was diluted $1: 10$ with $0.2 \underline{\mathrm{M}}$ ferulic acid (50:50 methanol/water). $100 \mu \mathrm{L}$ aliquots of this sample solution were cast directly onto $2 \times 2 \times 1 / 16^{n}$ quartz plates and dried under a gentle stream of room temperature air. A sample without dye and a sample without DNA were prepared in the same manner for use as controls.

Results

Although the signal to noise was rather poor, useful images of MALD generated DNA plumes were obtained. These results are presented in Figure 31. Here the plume 

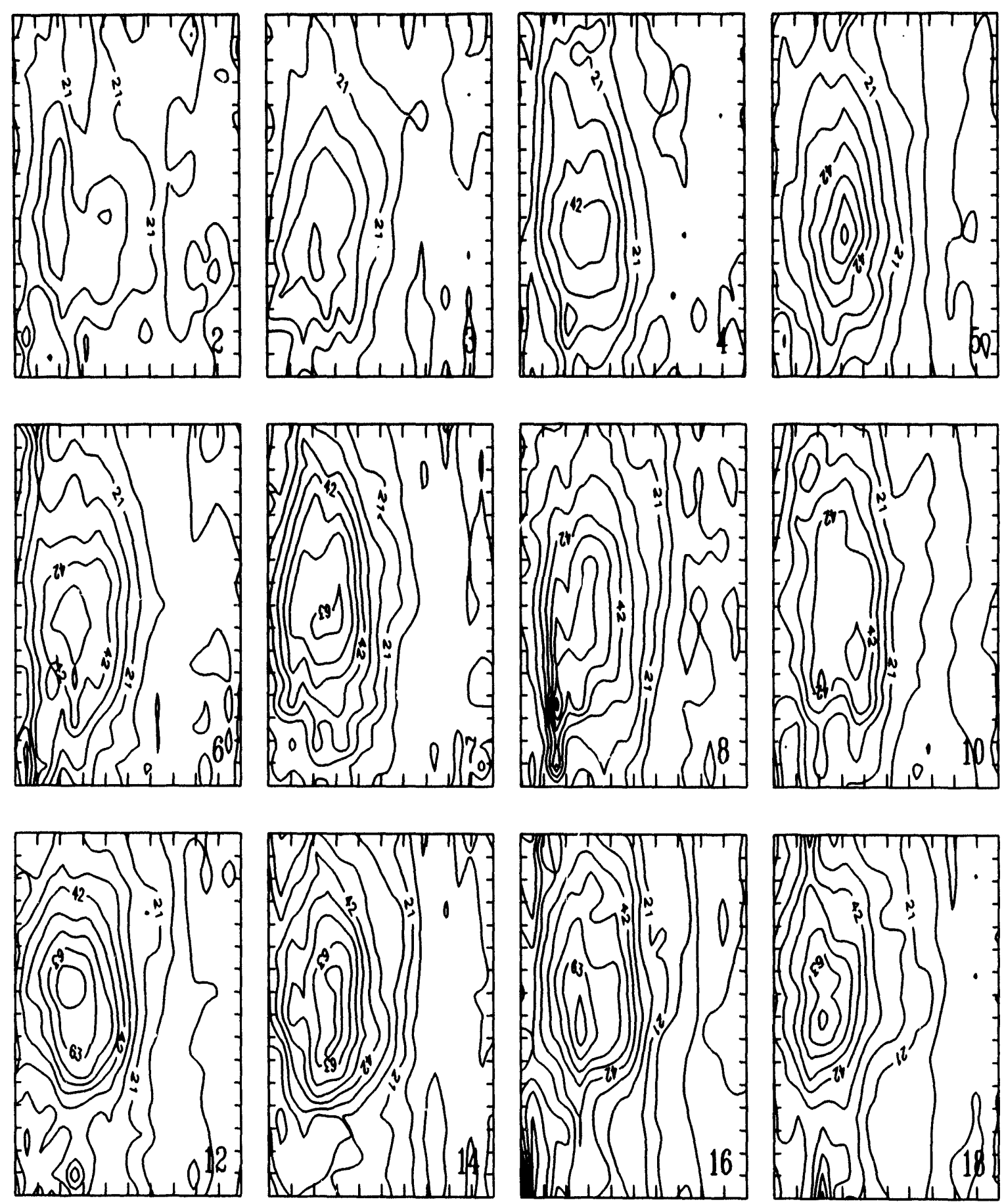

Figure 31. Selected sequential imar as of a MALD plume from DNA:TOTO-1 in a ferulic acid matrix 
appears to move away from the surface more rapidly than the protein. Although not likely, this may be an indication that the DNA:dye complex does not remain intact throughout the desorption process. Another possibility is that size of the molecule is a factor.

A PMT signal was observed at $520 \mathrm{~nm}$ from all samples, including unlabeled DNA, DNA:dye, and dye alone. This is likely due to incomplete discrimination against scattering of the probe laser. At 550 and $577 \mathrm{~nm}$ no signal was observed from unlabeled DNA. At $550 \mathrm{~nm}$ signal was seen from the DNA:dye complex only. At 57$\mathrm{nm}$ both the dye and the DNA:dye complex gave signals.

To help determine if dye-matrix interactions might be leading to dye fluorescence, fluorescence spectra of the various combinations were collected. Fluorescence intensities were determined for constant dye concentrations. Results are presented in Table VII. Measured fluorescence intensities confirmed that there was a fluorescence enhancement upon binding, but here it was closer to one order of

Table VII. Fluorescence intensities of TOTO-1 with various concomitants

\begin{tabular}{lc}
\hline sample & fluorescence intensity $(\mathrm{cps})$ \\
\hline TOTO-1 & 50,000 \\
TOTO-1/ferulic acid & 100,000 \\
TOTO-1/DNA/ferulic acid & 300,000 \\
TOTO-1/DNA & $1,000,000$ \\
\hline
\end{tabular}


magnitude than the three claimed by Molecular Probes. The presence of matrix doubled the fluorescence signal of dye, but quenched the complex.

Referring to Figure 32 one can see that the matrix also shifts the DNA:dye complex emission wavelength. Although it is possible that the DNA:dye complex is not preserved upon desorption, the fact that the DNA:dye plume yields a signal at $\mathbf{5 5 0}$ $\mathrm{nm}$ while dye alone does not strongly suggests that the complex remains intact. The fact that it might do so should not be surprising with the soft nature of MALD desorption. 


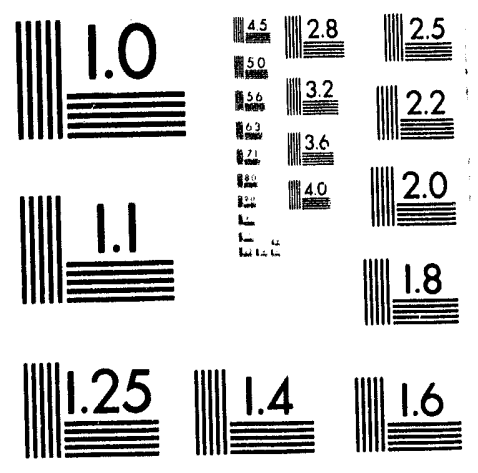



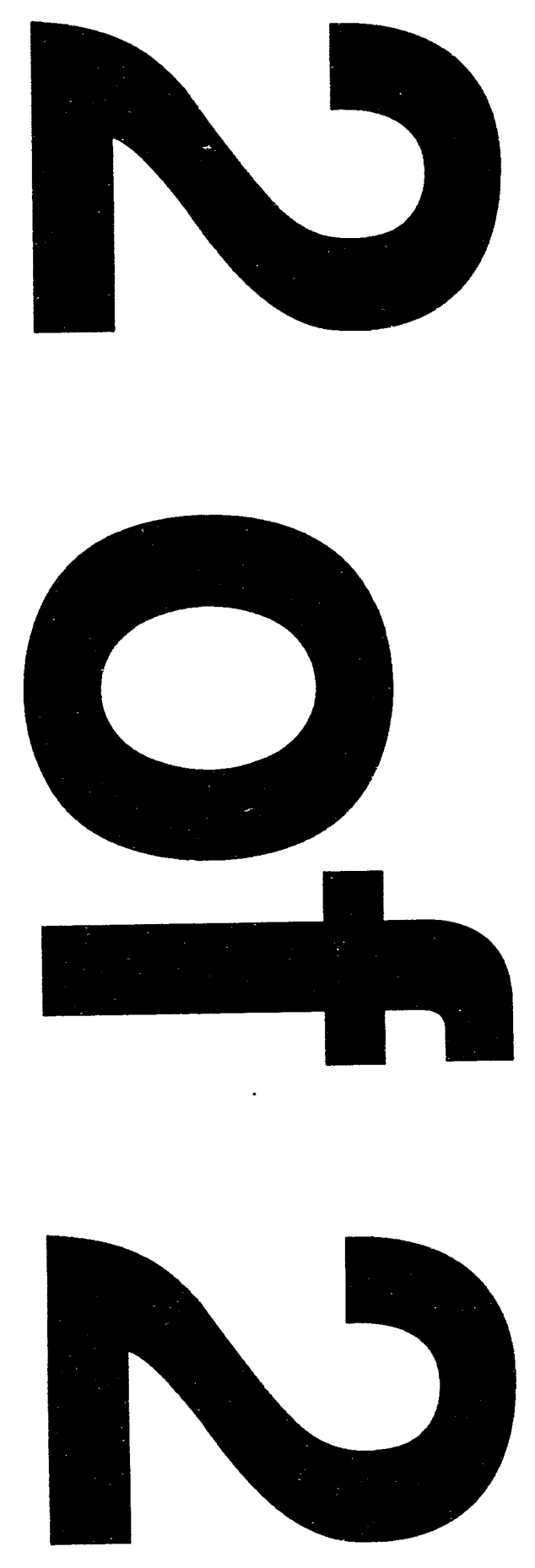


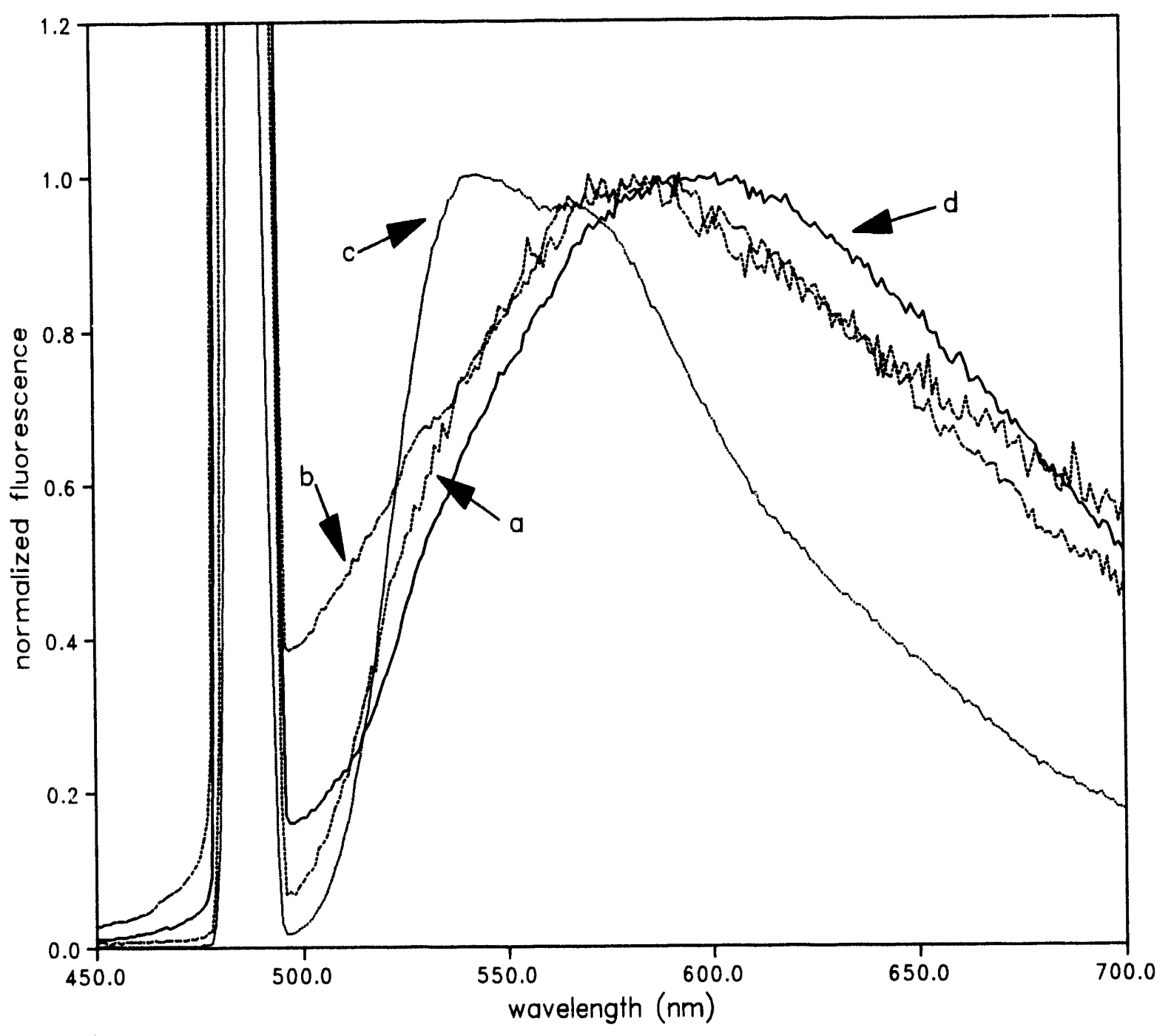

Figure 32. Normalized fluorescence spectra of a) TOTO-1; b) TOTO-1/ferulic acid c) DNA:TOTO-1 complex; d) DNA:TOTO-1/ferulic acid 


\section{CHAPTER 9. SUMMARY}

Matrix-assisted laser desorption is a technique for converting large biomolecules. into gas phase ions. Some characteristics of the commonly used UV matrices were determined including solubilities in methanol and solid phase absorption spectra. Acoustic and quartz crystal microbalance signals were investigated as possible means of UV-MALD quantitation. Evidence for the existence of desorption thresholds is presented. Threshold values were determined to be in the range of 2 to $3 \mathrm{MW} / \mathrm{cm}^{2}$. A transient imaging technique based on laser-excited fluorescence for monitoring MALD plumes is described. Sensitivity is well within the levels required for studying matrixassisted laser desorption, where analyte concentrations are significantly lower than those in conventional laser desorption. Results showing the effect of film morphology, particularly film thickness, on plume dynamics are presented. Fluorescent labeling of protein and of DNA is used to allow imaging of their UV-MALD generated plumes. The spatial and temporal distributions are important for the design of secondary ionization schemes to enhance ion yields and for the optimization of ion collection in time-of-flight instruments to maximize resolution.

Although the present study involves plumes in a normal atmosphere, adaptation to measurements in vacuum (e.g. inside a mass spectrometer) should be straightforward. Since the integrated concentration is available for each time segment, one can potentially assess the rate of desorption and the rate of fragmentation, ${ }^{96}$ if any. If in the future information about the spectroscopic differences between the 
neutral and the ionic forms become available, one can then determine the fraction of the desorbed analytes that are ionized at each point. Such information will provide insight into whether ionization is closely associated with the desorption step or whether it is a result of subsequent collisions with the matrix gas (e.g. proton transfer). Similar conclusions can be drawn about the mechanism of fragmentation.

The excitement surrounding MA-LD is primarily due to the ability to generate intact molecular ions from large proteins and oligonucleotides. It is hoped that this contribution will help in the effort to improve and to better understand the process. 


\section{REFERENCES CITED}

1. Maiman, T. H. Phys. Rev. Lett. 1960, 4, 564.

2. Kimbrell, S. M. Ph.D. Dissertation, Iowa State University, 1990.

3. Beckey, H. D. Principles of Field Ionization and Field Desorption Mass Spectrometry; Pergamon Press: Oxford, 1977.

4. Macfarlane, R. D.; Torgerson, D. F. Science 1976, 191, 920-925.

5. Barber, M.; Bordoli, R. S.; Sedgwick, R. S.; Tyler, A. N. J. Chem. Soc. Chem. Commun. 1981, 325-327.

6. Blakely, C. R.; Carmody, J. J.; Vestal, M. L. Anal. Chem. 1980, 52, 1636-1641.

7. Whitehouse, C. M.; Dreyer, R. N.; Yamashita, M.; Fenn, J. B. Anal. Chem. 1985, $57,675-679$.

8. Denoyer, E.; Van Grieken, R.; Adams, F.; Natusch, D. F. S. Anal. Chem. 1982, $54,26 \mathrm{~A}-41 \mathrm{~A}$.

9. Posthumus, M. A.; Kistemaker, P. G.; Meuzelaar, H. L. C.; Ten Noever de Brauw Anal. Chem. 1978, 50, 985-991.

10. Daves, G. Acc. Chem. Res. 1979, 12, 359-365.

11. Kimbrell, S. M.; Yeung, E. S. Appl. Spectrosc. 1991, 45(3), 442-447.

12. Karas, M.; Bachmann, D.; Hillenkamp, F. Anal. Chem. 1985, 57, 2935-2939.

13. Karas, M.; Bachmann, D.; Bahr, U. ;Hillenkamp, F. Int. J. Mass Spectrom. Ion Proc. 1987, 78, 53-68.

14. Tanaka, K.; Waki, H.; Ido, Y.; Akita, S.; Yoshida, Y.; Yoshida, T. Rapid Commun. Mass Spectrom. 1988, 2(8), 151-153.

15. Overberg, A.; Karas, M.; Hillenkamp, F. Rapid Commun. Mass Spectrom. 1991, 5, 128-131.

16. Overberg, A.; Karas, M.; Bahr, U.; Kaufmann, R.; Hillenkamp, F. Rapid Commun. Mass Spectrom. 1990, 4(8), 293-296.

17. Tang, K.; Allman, S. L.; Jones, R. B.; Chen, C. H. Org. Mass Spectrom. 1992, 27(12), 1389-1392. 
18. Cornett, D. S.; Duncan, M. A.; Amster, I. J. Org. Mass Spectrom. 1992, 27, 831832.

19. Beavis, R. C.; Chait, B. T. Rapid Commun. Mass Spectrom. 1989, 3(12), 436-439.

20. Annan, R. S.; Köchling, H. J.; Hill, J. A.; Biemann, K. Rapid Commun. Mass Spectrom. 1992, 6, 298-302.

21. Ens, W.; Mao, F.; Mayer, F.; Standing, K. G. Rapid Commun. Mass Spectrom. 1991, 5(3), 117-123.

22. Spengler, B.; Karas, M.; Bahr, U.; Hillenkamp, F. J. Phys. Chem. 1987, 91, 65026506.

23. Chevrier, M. R.; Cotter, R. J. Rapid Commun. Mass Spectrom. 1991, 5, 611-617.

24. Beavis, R. C.; Chait, B. T. Rapid Commun. Mass Spectrom. 1989, 3(12), 432-435.

25. Wu, K. J.; Steding, A.; Becker, C. H. Rapid Commun. Mass Spectrom. 1993, 7(2), 142-146.

26. Romano, L. J.; Levis, R. J. J. Am. Chem. Soc. 1991, 113, 9665-9667.

27. Chan, T.-W. D.; Colburn, A. W.; Derrick, P. J. Org. Mass Spectrom. 1992, 27, 5356.

28. Nelson, R. W.; Rainbow, M. J.; Lohr, D. E.; Williams, P. Science, 1989, 246, 1585-1587.

29. Spier, J. P.; Amster, I. J. Anal. Chem. 1992, 64(9), 1041-1045.

30. Beavis, R. C. Org. Mass Spectrom. 1992, 27, 653-659.

31. Spengler, B.; Kaufmann, R. Analusis, 1992, 20, 91-101.

32. Karas, M.; Bahr, U.; Gießmann, U. Mass Spectrom. Rev. 1991, 10, 335-357.

33. Ehring, H.; Karas, M.; Hillenkamp, F. Org. Mass Spectrom. 1992, 27, 472-480.

34. Beavis, R. C.; Chait, B. T. Rapid Commun. Mass Spectrom. 1989, 3(7), 233-237.

35. Castro, J. A.; Köster, C.; Wilkins, C. Rapid Commun. Mass Spectrom. 1992, 6, 239 241. 
36. Doroshenko, V. M.; Cornish, T. J.; Cotter, R. J. Rapid Commun. Mass Spectrom. 1992, 6(12), 753-757.

37. Schär, M.; Börnsen, K. O.; Gassmann, E. Rapid Commun. Mass Spectrom. 1991, 5, 319-326.

38. Beavis, R. C.; Chait, B. T. Proc. Natl. Acad. Sci. USA 1990, 87, 6873-6877.

39. Spengler, B.; Kirsch, D.; Kaufmann, R.; Jaeger, E. Rapid Commun. Mass Spectrom. 1992, 6, 105-108.

40. Beavis, R. C.; Chait, B. T. Anal. Chem. 1990, 62(17), 1836-1840.

41. Strupat, K.; Karas, M.; Hillenkamp, F. Int. J. Mass Spectrom. \& Ion Proc. 1991, 111, 89-102.

42. Doktycz, S. J.; Savickas, P. J.; Krueger, D. A. Rapid Commun. Mass Spectrom. 1991, 5, 145-148.

43. Salehpour, M.; Perera, I.; Kjellberg, J.; Hedin, A.; Islamian, M. A.; Håkansson, P.; Sundqvist, B. U. R. Rapid Commun. Mass Spectrom. 1989, 3(8), 259-263.

44. Mock, K. K.; Sutton, C. W.; Cottrell, J. S. Rapid Commun. Mass Spectrom. 1992, 6, 233-238.

45. Vertes, A.; Balazs, L.; Gijbels, R. Rapid Commun. Mass Spectrom. 1990, 4(7), 263266.

46. Sundqvist, B. U. R. Int. J. Mass Spectrom. Ion Proc. 1992, 118/119, 265-287.

47. Vertes, A.; Gijbels, R.; Levine, R. D. Rapid Commun. Mass Spectrom. 1990, 4, 228-233.

48. Johnson, R. E.; Sundqvist, B. U. R. Rapid Commun. Mass Spectrom. 1991, 5, 574578.

49. Vertes, A., In Methods and Mechanisms for Producing Ions from Large Molecules; K. G. Standing; W. Ens, Ed.; Plenum Press: New York, 1991; pp 275-286.

50. Williams, P.; Nelson, R. W., In Methods and Mechanisms for Producing Ions from Large Molecules; K. G. Standing; W. Ens, Ed.; Plenum Press: New York, 1991; pp 265-273. 
51. Beavis, R. C.; Chait, B. T., In Methods and Mechanisms for Producing Ions from Large Molecules; K. G. Standing; W. Ens, Ed.; Plenum Press: New York, 1991; pp 227-234.

52. Fain, B.; Lin, S. H. J. Chem. Phys. 1989, 91(4), 2726-2734.

53. Lindner, B.; Seydel, U. Anal. Chem. 1985, 57(4), 895-899.

54. Bitensky, I. S.; Goldenberg, A. M.; Parilis, E. S., In Methods and Mechanisms for Producing Ions from Large Molecules; K. G. Standing; W. Ens, Ed.; Plenum Press: New York, 1991; pp 287-291.

55. Conzemius, R. J.; Capellan, J. M. Int. J. Mass Spectrom. Ion Phys. 1980, 34, 197.

56. Hillenkamp, F., In Ion Formation from Organic Solids II; A. Benninghoven, Ed.; Springer-Verlag: New York, 1983; pp 190-205.

57. Steenvoorden, R. J. J. M.; Weeding, T. L.; Kistemaker, P. G.; Boon, J. J., In Methods and Mechanisms for Producing Ions from Large Molecules; K. G. Standing; W. Ens, Ed.; Plenum Press: New York, 1991; pp 315-323.

58. Hedin, A.; Westman, A.; Håkansson, P.; Sundqvist, B. U. R.; Mann, M., In Methods and Mechanisms for Producing Ions from Large Molecules; K. G. Standing; W. Ens, Ed.; Plenum Press: New York, 1991; pp 211-219.

59. Schlag, E. W.; Grotemeyer, J.; Levine, R. D. Chem. Phys. Letters 1992, 190(6), 521-527.

60. Pan, Y.; Cotter, R. J. Org. Mass Spectrom. 1992, 27, 3-8.

61. Hansen, S. G. J. Appl. Phys. 1989, 66, 3329-3336.

62. Beavis, R. C.; Chait, B. T. Chem. Phys. Letters 1991, 181(5), 479-484.

63. Zhou, J.; Ens, W.; Standing, K. G.; Verentchikov, A. Rapid Commun. Mass Spectrom. 1992, 6(11), 671-678.

64. Hillenkamp, F.; Karas, M.; Beavis, R. C.; Chait, B. C. Anal. Chem. 1991, 63(24), 1193A-1203A

65. Talbo, G.; Roepstorff,P. Rapid Commun. Mass Spectrom. 1993, 7(3), 201-204.

66. Lewis, S.; Korsmeyer, K. K.; Correia, M. A. Rapid Commun. Mass Spectrom. 1993, 7(1), 16-19. 
67. Stahl, B.; Steup, M.; Karas, M.; Hillenkamp, F. Anal. Chem. 1991, 63(14), 14631466.

68. Nelson, R. W.; Thomas, R. M.; Williams, P. Rapid Commun. Mass Spectrom. 1990, 4(9), 348-351.

69. Karas, M.; Bahr, U.; Ingendoh, A.; Hillenkamp, F. Angew. Chem. 1989, 101, 805806.

70. Spectrosc. 1992, 8(4), 26.

71. Houk, R. S., In Analytical Applications of Lasers; E. H. Piepmeier, Ed.; John Wiley \& Sons, Inc. New York, 1986; p 587-625.

72. Patel, R. S.; Brewster, M. Q. J. Heat Transfer, 1990, 112, 170-177.

73. Pearl, I. A.; Beyer, D. L. J. Org. Chem. 1951, 16, 216-220.

74. Nordhoff, E.; Ingendoh, A.; Cramer, R.; Overberg, A.; Stahl, B.; Karas, M.;

Hillenkamp, F.; Crain, P. F. Rapid Commun. Mass Spectrom. 1992, 6(12), 771-776.

75. Chen, G.; Yeung, E. S. Anal. Chem. 1988, 60(20), 2258-2263.

76. Pang, H.-M. ; Yeung, E. S. Anal. Chem. 1989, 61(22), 2546-2551.

77. Lai, Y.-M.; Cheung, N.-H. Rev. Sci. Instrum. 1993, submitted.

78. Leung, W. P.; Tam, A. C. Appl. Phys. Lett. 1992, 60(1), 23-25.

79. Buttry, D. A.; Ward, M. D. Chem. Rev. 1992, 92(6), 1355-1379.

80. Sauerbrey, G. Z. Z. Phys. 1959, 155, 206-222.

81. Pérez, J.; Weiner, B. R. Appl. Surf. Science 1992, 62, 281-285.

82. Hillier, A. C.; Ward, M. D. Anal. Chem. 1992, 64(21), 2539-2554.

83. Beavis, R. C. Org. Mass Spectrom. 1992, 27(8), 864-868.

84. Demirev, P.; Westman, A.; Reimann, C. T.; Håkansson, P.; Barofsky, D.;

Sundqvist, B. U. R.; Cheng, Y. D.; Seibt, W.; Siegbahn, K. Rapid Commun. Mass Spectrom. 1992, 6, 187-191.

85. Van Vaeck, L.; Van Roy, W.; Gijbels, R. Analusis, 1993, 21, 53-75. 
86. Becker, C. H., In Methods and Mechanisms for Producing Ions from Large Molecules; K. G. Standing; W. Ens, Ed.; Plenum Press: New York, 1991; pp 293296.

87. Kinsel, G. R.; Lindner, J.; Grotemeyer, J.; Schlag, E. W. J. Phys. Chem. 1991, 95, 7824-7830.

88. Iida, Y.; Morikawa, H.; Tsuge, A.; Uwamino, Y.; Ishizuka, T Anal. Sciences 1991, 7, 61-64.

89. Steenhoek, L. E.; Yeung, E. S. Anal. Chem. 1981, 53, 528-532.

90. Huie, C. W.; Yeung, E. S. Spectrochim. Acta 1985, 40B, 1255-1258.

91. Pang, H. M.; Yeung, E. S. Appl. Spectrosc. 1990, 44, 1218-1220.

92. Pang, H. M.; Yeung, E. S. Anal. Chem. 1989, 61, 2546-2551.

93. Tran, C. D. Anal. Chem. 1992, 64(20), 971A-981A.

94. Lekavich, J. Lasers \& Appl. 1986, 4, 59-64.

95. Heise, T. W.; Yeung, E. S. Anal. Chem. 1992, 64(18), 2175-2179.

96. Spengler, B.; Kirsch, D.; Kaufmann, R. Rapid Commun. Mass Spectrom. 1991, 5, 198-202. 


\section{ACKNOWLEDGEMENTS}

First and foremost I acknowledge the Higher Power in my life. Were it not for this Great Spirit, I would not be.

I thank my parents. Just saying thanks seems very inadequate for all they have given me, but is all I can do here.

I thank Professor Ed Yeung. In addition to financial support he has provided me with much guidance and patience. Ed has taught me a great deal and I am in his debt.

I thank everyone in the Yeung research group. It has been a terrific group to work in. Besides learning a lot, I had many great experiences. A special thanks to Dave McGregor. His special sense of humor and his caring friendship will be sorely missed.

Finally, I thank Kathy Robinson. Without her loving support the writing of this dissertation would have been unbearably discouraging.

This work was performed at Ames Laboratory under contract no. W-7405-eng82 with the U. S. Department of Energy. The work was supported by the Director of Energy Research, Office of Basic Energy Sciences. The United States government has assigned the DOE Report number IS-T 1657 to this thesis.

Additional support was provided as a fellowship by Iowa State University Liberal Arts and Sciences College. 

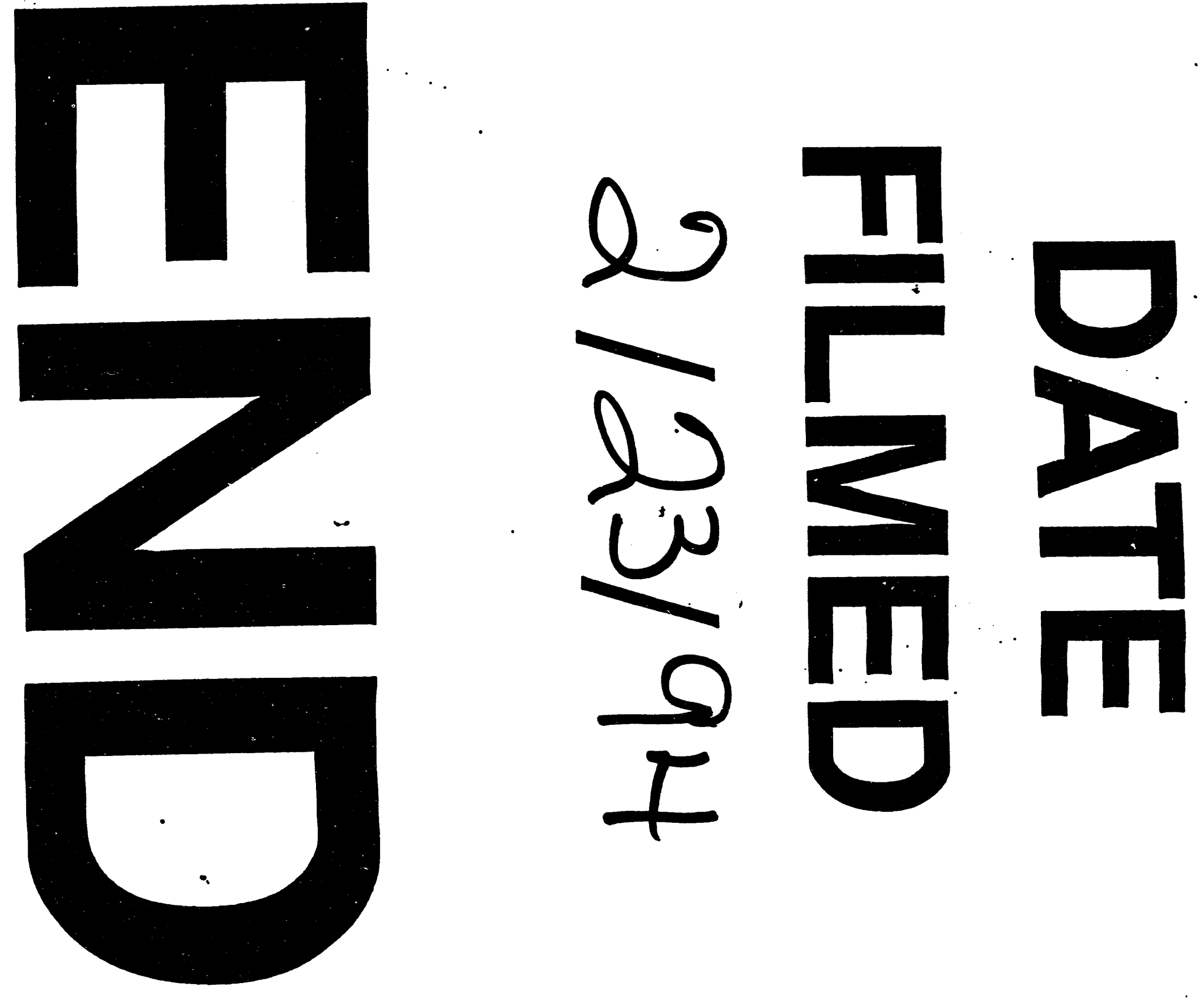